UNIVERSITÉ DU QUÉBEC À CHICOUTIMI

\author{
MÉMOIRE \\ PRÉSENTÉ À \\ L'UNIVERSITÉ DU QUÉBEC À CHICOUTIMI \\ COMME EXIGENCE PARTIELLE \\ DE LA MAÎTRISE EN SCIENCES INFIRMIÈRES
}

\author{
PAR \\ GENEVIÈVE TACHÉ \\ B. Sc. inf.
}

SIGNIFICATION DE L'EXPÉRIENCE VÉCUE DE PATIENTS EN ATTENTE DE TRANSPLANTATION VIVANT À L'EXTÉRIEUR DES GRANDS CENTRES DE LA PROVINCE DE QUÉBEC

AVRIL 2014 


\section{RÉSUMÉ}

L'attente d'une transplantation constitue un épisode complexe dans la vie des personnes qui ont à faire face à l'insuffisance d'un organe pour laquelle la greffe peut s'avérer une option thérapeutique. De plus, de nombreux chamboulements physiques et psychologiques surviennent lors de cette attente. À ce jour, très peu d'études ont donné une voix à ceux qui ont vécu l'attente d'une transplantation en considérant toutes les dimensions de leur expérience. Le but de cette étude phénoménologique descriptive était d'explorer l'expérience vécue de personnes en attente de transplantation au Québec, afin d'arriver à mieux comprendre cette réalité. Les sept participants recrutés se sont exprimés librement lors des entretiens et ont rapporté ce qu'ils ont vécu en attendant leur transplantation. Les témoignages recueillis ont été analysés selon la méthode de Colaizzi et le logiciel NVivo 10 a été utilisé pour faciliter la codification des propos recueillis.

L'expérience vécue au regard du phénomène à l'étude a été décrite à l'aide des trois thèmes qui ont émergé des analyses soit la volonté de vivre, garder l'espoir de vivre malgré la tourmente de l'incertitude et mettre sa vie en suspens. Lors de l'annonce de l'issue fatale de leur maladie, et ce, à plus ou moins brève échéance, les participants ressentent un choc important. Ils sont alors confrontés à l'obligation de prendre une grave décision : celle de tenter ou non la transplantation. Alors que pour certains, cette décision est prise d'emblée puisqu'elle constitue la seule avenue possible pour continuer à vivre, d'autres sont confrontés à un profond dilemme. Les résultats de cette étude font également ressortir que lorsque cette décision est prise, les patients doivent affronter une réalité à laquelle ils ne s'attendaient pas. L'espoir de vivre, motivation intrinsèque qui leur permet de poursuivre leur attente, est soumis à de nombreuses épreuves. Ainsi, l'omniprésence de la mort, la solitude, une constante incertitude et le sentiment que le temps s'est arrêté et que leur vie est mise en suspens hantent le quotidien des patients en attente de transplantation. Ils développent donc des stratégies d'adaptation afin de conserver cet espoir de vivre.

Le présent mémoire vise à élargir la compréhension du phénomène de l'attente d'une transplantation et suggère des recommandations pour la pratique, la recherche et la formation infirmière.

Mots clés: attente, transplantation d'organe, greffe d'organe, recherche qualitative, phénoménologie 


\section{REMERCIEMENTS}

La route qui mène à la maîtrise est parsemée d'embûches, de travail acharné et de sacrifices. Par contre, cette réalisation représente une victoire et constitue une fierté personnelle. Cette réussite a été possible grâce au soutien et à l'accompagnement de madame Suzanne Aucoin, inf., Ph.D., directrice de mémoire. Elle a su me guider tout au long de cette aventure. Sa compréhension et son appui dans les moments plus difficiles en font une personne d'exception pour moi. Elle est une source d'inspiration et de dévouement. Je tiens également à remercier sincèrement madame Édith Ellefsen inf., Ph.D., codirectrice, qui a accepté avec empressement de faire partie de cette aventure. Son expertise en recherche qualitative phénoménologique s'est avérée une aide précieuse qui m'a permis de mieux saisir et, je l'espère, de mieux traduire ce que représente l'essence de l'expérience vécue des personnes en attente de greffe.

Je remercie également madame Micheline Cyr Asselin, qui m'a ouvert les portes de la Maison des greffés du Québec. De même, mes remerciements vont à la personne responsable de 1'Association régionale des greffés du Saguenay-Lac-Saint-Jean, pour avoir spontanément accepté de me mettre en contact avec les membres de son association. Sans eux, le recrutement aurait sans doute été encore plus difficile, voire impossible. 
Je dois également remercier du fond du cœur mon mari, Dimitri Gagné, pour sa compréhension, son soutien et ses encouragements de tous les instants. Il a su prendre en charge tout ce qu'une maman qui travaille et étudie n'a pas le temps de faire, et ce, tout en assumant ses propres tâches. Merci également à ma petite princesse, ma belle Alice, pour le temps que je n'ai pu passer avec elle parce que je devais travailler sur mon projet.

Je ne peux passer sous silence l'appui inconditionnel de mes parents, Germain et Francine, qui m'ont également apporté une aide précieuse durant mes études. Je tiens à exprimer un merci tout particulier à mon père, Germain Taché, un homme d'exception, dont la condition de santé a inspiré ce projet de recherche. Merci de m'avoir appris à garder espoir en la vie et à lui faire confiance. Ta force et ton courage face à l'adversité sont pour moi une source d'inspiration et le demeureront à jamais. 


\section{TABLE DES MATIÈRES}

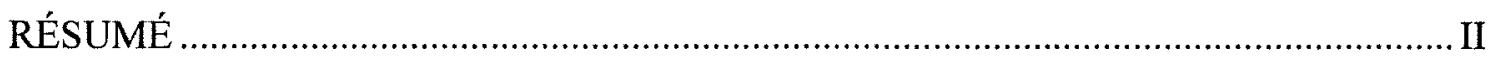

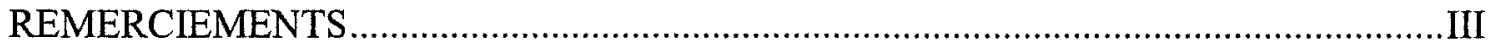

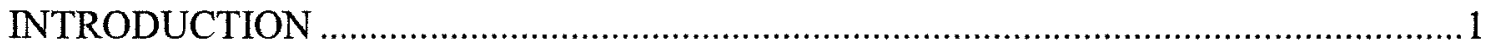

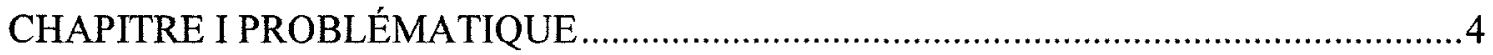

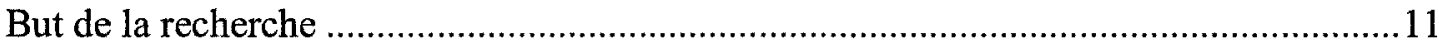

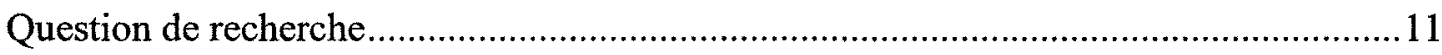

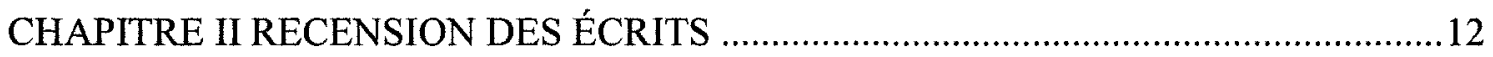

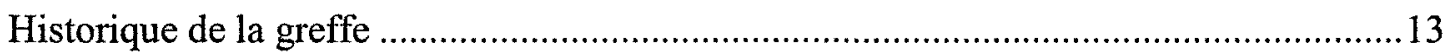

La maladie chronique terminale .............................................................................. 14

Nécessité d'une transplantation .................................................................................16

L'expérience d'attendre une transplantation..............................................................17

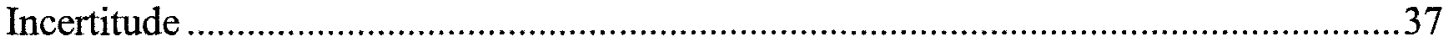

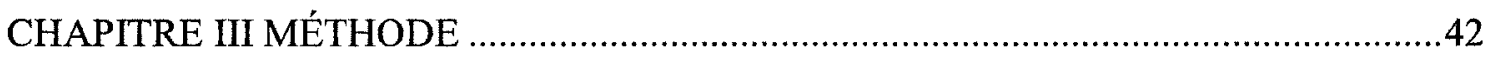

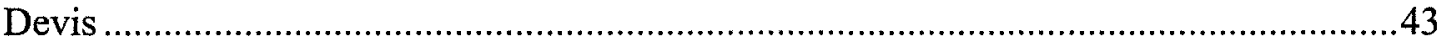

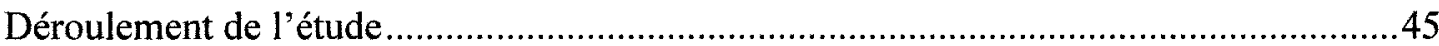

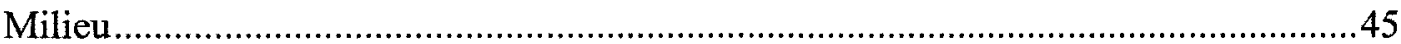

Participants à l'étude ............................................................................................46

Outils de collecte de données...................................................................................49

Questionnaire sociodémographique.......................................................................49

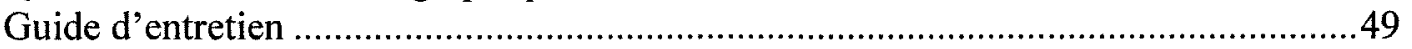

Collecte de données ..........................................................................................

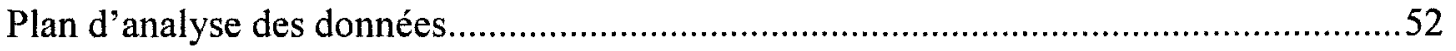

Critères de rigueur en analyse qualitative.................................................................54

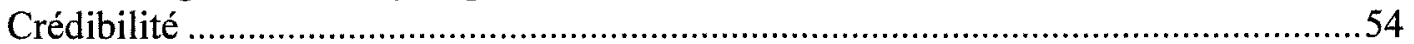

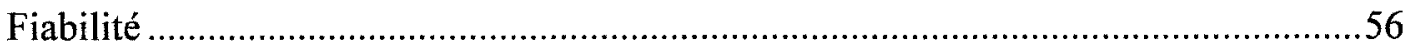

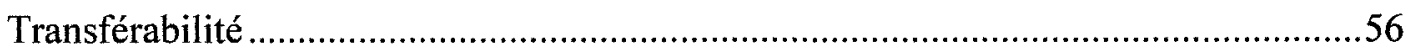

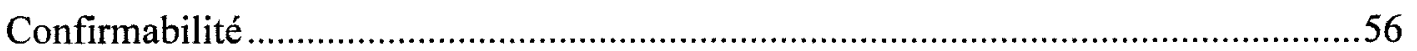

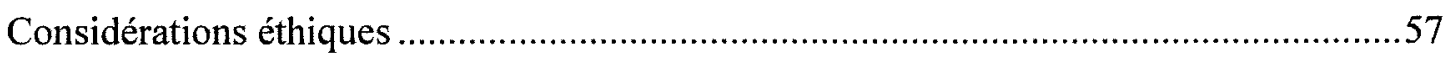

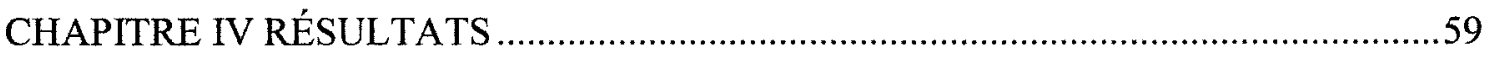

Données sociodémographiques et cliniques des participants ..........................................60

L'expérience d'attendre une transplantation...................................................................62

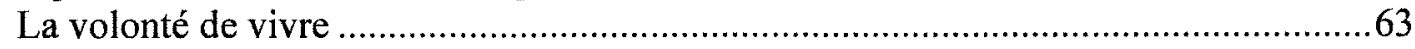

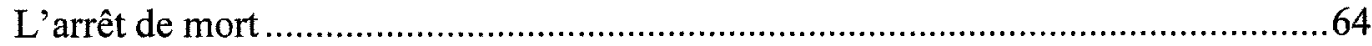

La prise de décision de continuer à vivre ............................................................66

Garder l'espoir de vivre malgré la tourmente de l'incertitude......................................68 


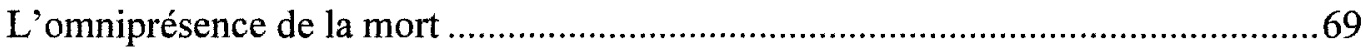

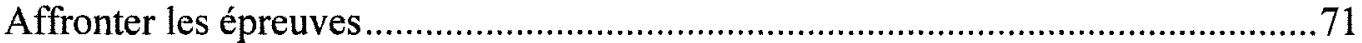

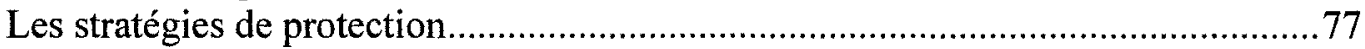

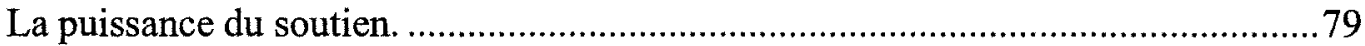

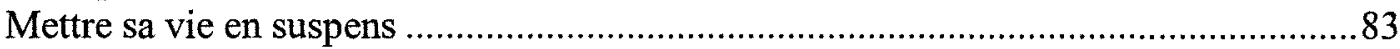

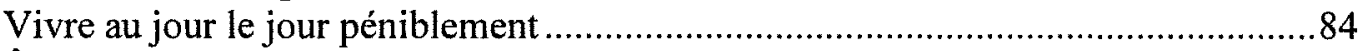

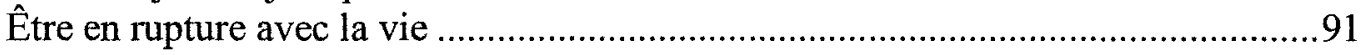

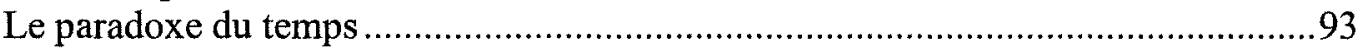

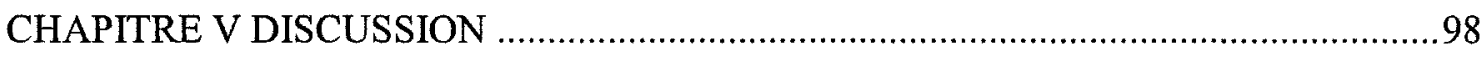

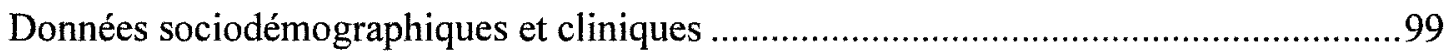

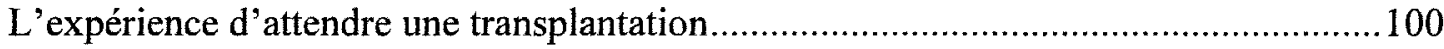

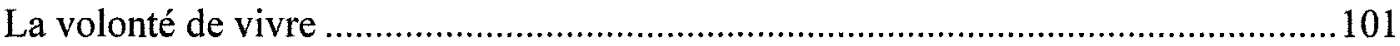

Garder l'espoir de vivre malgré la tourmente de l'incertitude...................................105

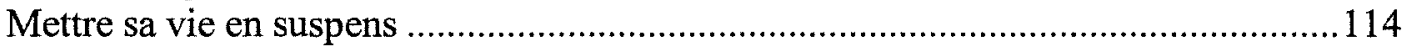

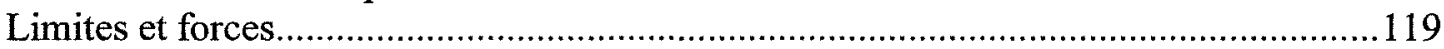

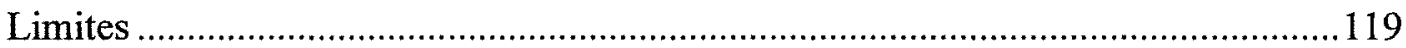

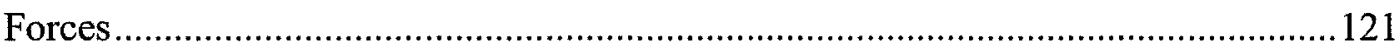

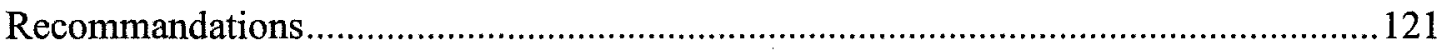

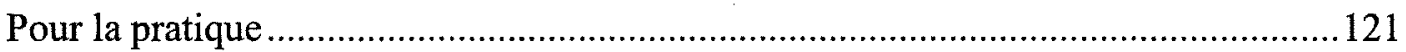

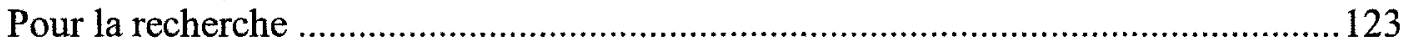

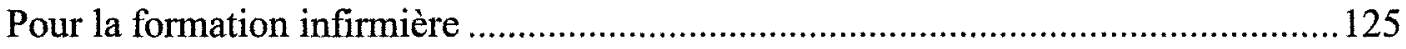

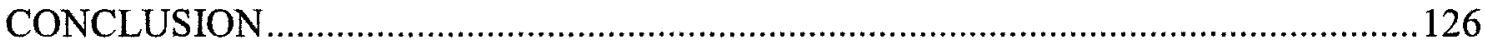

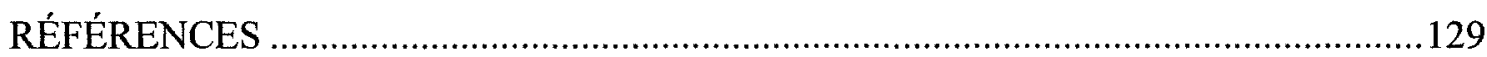

APPENDICE A LETTRE DE DEMANDE D'AUTORISATION DE RECRUTEMENT ET D'INFORMATION SUR LE DÉROULEMENT DE LA COLLECTE DE DONNÉES AUPRÈS DE LA MAISON DES GREFFÉS DU QUÉBEC ....................................136

APPENDICE B AUTORISATION DE RECRUTEMENT À LA MAISON DES GREFFÉS DU QUÉBEC..

APPENDICE C APPROBATION ÉTHIQUE DE L'UNIVERSITÉ DU QUÉBEC À CHICOUTIMI

APPENDICE D LETTRE D'INFORMATION SUR LE PROJET DE MÉMOIRE DE MAÎTRISE POUR LES PROFESSIONNELS DU PROGRAMME DE

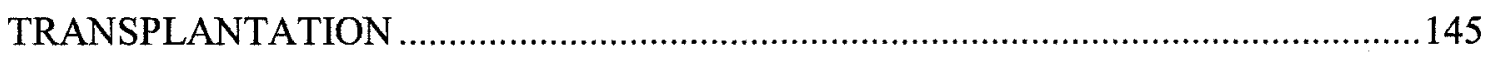

APPENDICE E AFFICHE DE RECRUTEMENT ................................................ 148

APPENDICE F LETTRE D'INFORMATION SUR LE PROJET DE MAÎTRISE .........150

APPENDICE G QUESTIONNAIRE SOCIODÉMOGRAPHIQUE ...........................153

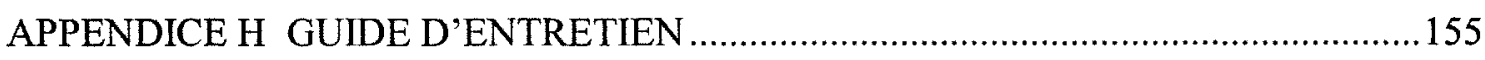


APPENDICE I FORMULAIRE D'INFORMATION ET DE CONSENTEMENT CONCERNANT LA PARTICIPATION.

APPENDICE J ACCEPTATION DU SUIVI PSYCHOLOGIQUE PAR LES ÉQUIPES DE TRANSPLANTATION

APPENDICE K RÉSUMÉ DU JOURNAL DE BORD DE L'ÉTUDIANTE CHERCHEURE.

APPENDICE L ACCEPTATION DE MODIFICATION PAR LE COMITÉ D'ÉTHIQUE DE LA RECHERCHE DE L'UNIVERSITÉ DU QUÉBEC À CHICOUTIMI. 
INTRODUCTION 
La transplantation représente, pour certains patients dont l'un des organes vitaux a cessé de fonctionner, le seul espoir de prolonger leur vie. Alors que le nombre de donneurs d'organes décroit année après année au Québec, les besoins en termes de greffes d'organe augmentent continuellement et le temps d'attente s'allonge d'autant plus pour les patients en attente de transplantation (Transplant Québec, 2013c). Cette période s'avère un moment difficile et lourd d'incertitude, compte tenu des taux élevés de morbidité et de mortalité qui y sont associés (Transplant Québec, 2013c). Malgré les sentiments initiaux de joie et de gratitude qui accompagnent le fait d'être placé sur la liste d'attente pour une transplantation, l'anxiété et l'instabilité émotionnelle font surface rapidement lors de la période de l'attente. Pour réussir à y faire face, la personne qui attend doit alors recourir à des stratégies d'adaptation afin de rendre plus tolérable la vie quotidienne pendant cette période plus ou moins longue (Brown, Sorrell, McClaren, \& Creswell, 2006).

L'expérience de l'attente d'une transplantation a fait l'objet de quelques études soit en utilisant un devis qualitatif (Bjork, \& Naden, 2008; Brown et al., 2006; Haugh, \& Salyer, 2007; Jonsén, Athlin, \& Suhr, 2000; Macdonald, 2006; Moran, Scott, \& Darbyshire, 2011; Naef, \& Bournes, 2009; Sadala, \& Stolf, 2008), quantitatif (Ashcroft, 2009; Martin, Stone, Scott, \& Brashers, 2010; Telles-Correia, Barbosa, Mega, \& Monteiro, 2009) ou en réalisant une revue des publications scientifiques, particulièrement sur les divers impacts que peut générer l'attente chez le patient (McDermott, Hardy, \& McCurry, 2010). Cependant, les études relatives à l'expérience vécue des personnes en attente de greffe sont encore parcellaires et nettement incomplètes. 
Ce mémoire comporte cinq chapitres. Le premier met en lumière les éléments qui contribuent à la problématique étudiée, puis expose le but et la question de recherche. Le second chapitre propose une recension des écrits qui rapporte les résultats de différentes recherches réalisées sur le sujet au cours des dernières années. Il débute par un bref historique de la greffe et décrit ce que vivent les patients atteints d'une maladie terminale et qui sont susceptibles de recevoir une transplantation. Il fait également état de ce que représente l'attente d'une transplantation et aborde le concept d'incertitude. Le troisième chapitre comprend la description de la méthode, les critères de rigueur scientifique à respecter dans une étude qualitative et les considérations éthiques. Le quatrième chapitre rapporte les résultats de cette recherche alors que le cinquième et dernier chapitre présente la discussion des résultats, les limites et les forces de l'étude, les recommandations pour la pratique, la recherche et la formation infirmière. Une brève conclusion termine ce mémoire. 


\section{CHAPITRE I}

PROBLÉMATIQUE 
L'annonce du diagnostic d'une maladie grave à évolution fatale à plus ou moins court terme constitue un moment très difficile pour le patient. Il prend alors conscience que sa vie est bouleversée et que plus rien ne sera désormais pareil (Bjork, \& Naden, 2008). Cependant, au début de la maladie, il peut généralement compter sur sa famille, ses collègues de travail, ses amis et sur l'énergie physique qui est parfois encore présente. Par contre, lorsque le pronostic de la maladie chronique s'assombrit et que la greffe d'organe devient l'option de choix, le patient a souvent épuisé tout ce qu'il avait de ressources et d'énergie (Bjork, \& Naden, 2008). La diminution de la qualité de vie (Limbos, Joyce, Chan, \& Kesten, 2000) et la détresse psychologique sont alors bien présentes (Corruble et al., 2010). De plus, des problèmes familiaux et conjugaux peuvent apparaître, auxquels s'ajoutent, dans la majorité des cas, des ennuis financiers, puisque la personne malade s'avère souvent celle qui subvenait aux besoins de la famille et ne peut désormais plus travailler, compte tenu de son état de santé (McDermott et al., 2010).

Cependant, les personnes atteintes d'une maladie terminale ne sont pas toutes éligibles à la greffe. Ainsi, l'admissibilité à la transplantation doit être scrupuleusement évaluée par les professionnels ${ }^{1}$ cuurant dans les divers centres transplanteurs du Québec. Chaque candidat doit subir des examens médicaux et psychologiques qui déterminent si la greffe constitue l'option à privilégier dans sa situation et si celle-ci peut être réalisée avec succès (Baran, 2008). Entre autres, l'équipe de greffe doit s'assurer que la problématique de santé qui a conduit à la situation précaire du patient ne viendra pas détruire l'organe une fois la greffe réalisée (Brown et al., 2006).

\footnotetext{
${ }^{1}$ Le masculin est utilisé à titre épicène dans ce mémoire.
} 
Quant à l'évaluation psychologique, elle vise à estimer la présence de facteurs de risque, à intervenir en conséquence (Achille, 2002) et à s'assurer que le patient sera en mesure d'affronter les diverses embûches rencontrées tout au long de la période d'attente (Moloney, Cicutto, Hutcheon, \& Singer, 2007). Les évaluations réalisées doivent déboucher sur une approbation pour la transplantation et ce n'est qu'une fois le patient considéré prêt par les professionnels qu'il est placé sur une liste d'attente (Flamme, Terry, \& Helft, 2008).

L'inscription sur la liste d'attente pour une transplantation induit donc des situations qui exposent le candidat à des sources de stress et d'anxiété jusque-là inconnues telles la concurrence avec d'autres candidats eux aussi sur la liste d'attente ou la perte d'espoir lors du décès de l'un d'entre eux, la confrontation à sa propre mort à plus ou moins court terme, la conscience que quelqu'un doit mourir pour qu'il y ait un donneur, la diminution du réseau social, des tensions conjugales et une attente qui perdure (Achille, 2002). L'attente constitue donc une période fort difficile pour le patient puisque l'incertitude, la dépression, la diminution de la qualité de vie, un sentiment de perte de contrôle et même de la culpabilité peuvent découler du stress intense généré par sa condition physique (Young, Molzahn, Starzomski, \& Budz, 2010).

Cette incertitude, qui fait partie intégrante du quotidien du patient vivant avec une maladie en phase terminale en attente de transplantation (Lasker, Sogolow, Olenik, Sass, \& Weinrieb, 2010) est souvent liée aux conséquences de la maladie, à la complexité du traitement, au manque d'information sur le degré de sévérité de la maladie et, finalement, au pronostic de celle-ci. Sa présence peut contribuer également à majorer le niveau de 
stress et générer elle aussi de l'anxiété de même que de la dépression (Kurz, \& Cavanaugh, 2001), occasionner une diminution de la tolérance à la douleur, de la capacité d'adaptation ainsi que de la qualité de vie (Lasker et al., 2010).

Le stress éprouvé peut être lié à de multiples facteurs dont les préoccupations financières et la nécessité pour certains d'être hébergés loin de chez eux pour être à proximité du centre transplanteur (Kurz, \& Cavanaugh, 2001). De plus, le manque de ressources et d'information en ce qui concerne les différentes étapes à franchir entre l'attente et la greffe sont des éléments d'incertitude importants qui contribuent à l'augmentation du stress chez les patients en attente (Kurz, \& Cavanaugh, 2001; Mishel, 1988). Les conséquences du stress sur leur état de santé peuvent être suffisamment importantes pour les empêcher de recevoir leur organe et parfois même entraîner la mort (Corruble et al., 2010). Cette attente est définie par les patients comme une période d'angoisse et d'adaptation très difficile (Burker et al., 2006).

$\mathrm{Au}$ Québec, cette situation se complexifie davantage lorsque le patient habite à l'extérieur des grands centres, soit Montréal et Québec, puisque les établissements qui offrent la transplantation se situent uniquement dans ces deux grandes villes (Transplant Québec, 2013b). Compte tenu du temps de conservation de l'organe une fois prélevé (Brown et al., 2006) et de la période nécessaire au déplacement du patient vers le centre de greffe, ce dernier peut donc se voir obligé de demeurer à proximité de son centre transplanteur. En 2012, la grande majorité des personnes en attente, soit 740 personnes sur 1250 (59,2\%), vivaient à l'extérieur de ces grands centres (Transplant Québec, 2013c). 
Selon les données de Transplant Québec (2013a), le temps de conservation est différent pour chaque organe, soit de quatre à six heures pour un cœur et des poumons, six à 12 heures pour un pancréas, 12 à 16 heures pour un foie et 24 à 48 heures pour des reins. De ce fait, un patient qui réside en région éloignée et qui doit par exemple recevoir une transplantation cardiaque dans la région de Montréal devra trouver un hébergement dans cette ville puisque le temps de conservation d'un cœur est de quatre heures. La relocalisation des candidats à la transplantation et souvent de leur aidant naturel génère toutefois plusieurs problèmes tels éprouver le sentiment de mettre sa vie en attente, connaître une diminution du soutien affectif, devoir établir un nouveau réseau de soutien, subir des changements de rôle et être confronté à des préoccupations financières (Stubblefields, \& Murray, 2002). Cette situation s'avère majorée par le temps d'attente qui peut parfois durer plus de deux ans selon le type d'organe à recevoir (Transplant Québec, 2013c).

Le faible taux de don d'organe peut malheureusement expliquer le temps d'attente qui ne cesse d'augmenter avec les années. Le temps d'attente dépend du type d'organe à recevoir; ainsi, les patients en attente de transplantation cardiaque peuvent attendre en moyenne 189 jours, en attente de poumons 770 jours et de reins environ 1220 jours, soit près de quatre ans (Transplant Québec, 2013c). Le temps d'attente influence grandement l'expérience d'attendre une transplantation. Plus le temps d'attente est long et plus l'incertitude, l'anxiété et la détérioration de l'état de santé risquent d'augmenter (Bjork, \& Naden, 2008). Tout au long de cette période, les candidats vont vivre du découragement, de la frustration et de l'ennui. De plus, les patients finissent par croire que la greffe est hors de 
portée et certains iront même jusqu'à remettre en question la pertinence de la greffe (Brown et al., 2006).

En 2011, le Canada se retrouvait en dernière position pour le taux de don d'organe $(15,4 \%)$, comparativement à ceux comptabilisés en Espagne $(36,9 \%)$, aux États-Unis $(25,9 \%)$ et en France $(23,9 \%)$ (Transplant Québec, 2013c). Une des raisons qui contribue à l'obtention de ce faible pourcentage est l'identification inadéquate des donneurs potentiels. En effet, Transplant Québec (2013c) rapporte qu'environ $20 \%$ des donneurs d'organes ne sont pas identifiés. Un donneur potentiel correspond à une personne de tout âge, atteinte de lésions neurologiques graves et irréversibles, nécessitant une ventilation mécanique. Le donneur le plus âgé avait 88 ans et le plus jeune seulement 48 heures (Lavigne, 2012).

Au Québec, quelques associations et organismes viennent en aide aux futurs receveurs et à leur famille tels l'Association des greffés, la Fondation Diane Hébert et la Fondation Lina Cyr. À Montréal, une résidence a été ouverte pour permettre aux patients qui vivent en région éloignée d'être hébergés durant leur attente, la Maison des greffés du Québec. De plus, chaque centre hospitalier transplanteur peut compter sur la présence d'une équipe de greffe qui soutient le patient et ses proches lors de l'attente. De manière plus particulière, un infirmier pivot fait le lien entre les professionnels de l'équipe de greffe et le patient. Le rôle de ces derniers s'avère primordial dans le long parcours de la transplantation puisque le soutien fourni influencera la décision du patient de tenter ou non la greffe (Moloney et al., 2007). 
L'accompagnement de l'équipe de transplantation apparait indispensable à la sécurité du patient et à la réussite de sa greffe (Ashcroft, 2009). En effet, la compréhension des auto-soins par les patients et leur famille améliore les chances de survie du greffon. Avant que les patients soient inscrits sur la liste d'attente, ils doivent comprendre ce qu'implique d'être accepté sur cette liste, la transplantation comme telle de même que ce que sera la vie après une greffe. Il est donc essentiel que les soignants aident les patients à concevoir et à intégrer ce processus complexe (Ashcroft, 2009). À ce propos, la contribution des infirmières s'avère nécessaire pour soutenir le patient adéquatement lors de l'attente d'une transplantation puisque leurs interventions influencent directement la réaction des patients lors du processus de l'attente (Ashcroft, 2009).

Par ailleurs, la transplantation demeure un phénomène relativement nouveau et peu de recherches ont porté à ce jour sur ce que vivent les patients durant la période d'attente (Bjork, \& Naden, 2008; Brown et al., 2006; Haugh, \& Salyer, 2007; Jonsén et al., 2000; Limbos et al., 2000; Macdonald, 2006; Naef, \& Bournes, 2009; Sadala, \& Stolf, 2008; Telles-Correia et al., 2009). Dans les écrits recensés, les éléments d'information sur les problèmes réels rencontrés par les patients en attente de transplantation et sur leurs besoins sont parcellaires, et ce, malgré l'emphase mise sur l'importance de reconnaître l'impact de leur état émotionnel et psychologique lors de l'attente (Limbos et al., 2000) et sur la nécessité de répondre à leurs besoins (Ashcroft, 2009; Diaz-Dominguez, Perez-Bernal, Perez-San-Gregorio, \& Martin-Rodriguez, 2006).

Ainsi, vivre dans l'attente de recevoir un organe affecte grandement l'expérience de l'individu dans plusieurs sphères de sa vie (Brown et al., 2006). Les conséquences socio- 
économiques considérables et l'impact majeur sur la qualité de vie de ces personnes (Limbos et al., 2000) en font une clientèle vulnérable à plusieurs égards. En dépit des interventions entreprises dans les milieux cliniques, la présence de nombreuses difficultés rencontrées lors de l'attente d'une transplantation justifie la nécessité de mieux comprendre l'expérience vécue des patients en attente de transplantation.

\section{But de la recherche}

Le phénomène à l'étude est l'expérience de l'attente de transplantation. De manière plus particulière, le but de cette recherche consiste à décrire et comprendre la signification de l'expérience vécue de patients en attente de transplantation vivant à l'extérieur des grands centres de la province de Québec.

\section{Question de recherche}

Ce projet de recherche vise à répondre à la question suivante: Quelle est la signification de l'expérience d'attendre une transplantation?

Une fois la problématique mise en place, le chapitre suivant propose une recension des principaux écrits visant à étayer celle-ci. 


\section{CHAPITRE II}

RECENSION DES ÉCRITS 
Ce chapitre aborde la recension des écrits réalisée afin de mettre en lumière et d'approfondir les éléments présentés dans la problématique. La première partie décrit l'historique de la transplantation et expose le contexte de la maladie chronique terminale. La seconde partie aborde le vécu du patient en attente de transplantation, à savoir l'annonce de la nécessité de la transplantation et le processus d'attente. Enfin, la définition de la notion d'incertitude conclut ce chapitre.

\section{Historique de la greffe}

La greffe peut parfois constituer la seule et ultime option thérapeutique d'une maladie terminale et le dernier recours dans un parcours difficile, projetant alors le patient dans une situation d'invalidité et d'angoisse de la mort (Bjork, \& Naden, 2008). Ainsi, pour ceux qui sont atteints d'insuffisance rénale, pulmonaire ou cardiaque, de la fibrose kystique et de nombreuses autres pathologies, la transplantation représente la seule possibilité de survie (Baran, 2008).

Les premières greffes d'organes ont eu lieu au $\mathrm{XVIII}^{\mathrm{e}}$ siècle (Transplant Québec, 2013a). À partir de ce moment, un espoir de guérison a désormais été possible pour des patients qui, avant l'avènement de la greffe, n'avaient aucune chance de survie. La transplantation est une chirurgie pendant laquelle un organe ou un tissu sain d'un donneur est implanté chez un receveur. Le but de la transplantation est de remplacer un organe devenu non fonctionnel suite à un processus pathologique aigu ou chronique irréversible par un organe sain provenant d'un autre individu (Beyeler, \& Riggenbach, 2012).

Les techniques chirurgicales permettant d'opérer ce transfert d'organe d'un corps à l'autre ont été développées autour de 1905, mais les tentatives des pionniers de la greffe, 
notamment à Lyon, se sont soldées par le rejet de l'organe greffé par son hôte, phénomène alors incompris (Biomédecine, 2010). Au Québec, c'est en 1958 que la première greffe rénale provenant d'un donneur vivant a eu lieu à l'Hôpital Royal Victoria de Montréal (Lavigne, 2012). Toutefois, ce n'est qu'au début des années 1980 que la cyclosporine a fait son apparition et contribué, par son action immunosuppressive, à la survie des patients ayant subi une greffe d'organe (Beyeler, \& Riggenbach, 2012).

Au Canada, la première transplantation pulmonaire simple a été réalisée en 1983 et la première transplantation pulmonaire double en 1986. Depuis, les résultats pour les receveurs n'ont cessé de s'améliorer en raison notamment, de l'évolution des techniques chirurgicales, de méthodes de préservation des organes plus efficaces, de meilleurs soins pré et postopératoires, d'une meilleure prise en charge médicale des receveurs ainsi que des avancées dans le secteur de l'immunosuppression (Junius, 2010).

\section{La maladie chronique terminale}

Plusieurs auteurs affirment que les patients susceptibles de recevoir une transplantation d'organe sont généralement ceux vivant avec une maladie chronique en phase terminale, réfractaires aux traitements chirurgicaux et médicaux, dont la qualité de vie est diminuée (Bjork, \& Naden, 2008; Brown et al., 2006) et qui n'ont comme seule option de survie que la transplantation d'organe (Achille, 2002; Baran, 2008; Bjork, \& Naden, 2008; Brown et al., 2006; Young et al., 2010). Les maladies chroniques sont des problèmes de santé incurables et qui provoquent des symptômes ou des incapacités qui se prolongent dans le temps (Santé et Services sociaux du Québec, 2005). Elles sont devenues la principale cause de mortalité chez les patients en phase terminale (Cazale, 
Laprise, \& Nanhou, 2009). En plus du risque de mortalité, elles entraînent également des répercussions importantes dans plusieurs sphères de la vie des personnes qui en souffrent.

La maladie chronique génère particulièrement de l'anxiété chez le patient, car vivre pendant de nombreuses années avec des symptômes et les incapacités qui en découlent peut porter atteinte à l'identité, mener à des changements de rôles, altérer l'image corporelle et perturber les habitudes de vie (Habraken, Pols, Bindels, \& Willems, 2008). Vivre avec une maladie chronique, c'est vivre dans l'incertitude de ne pas savoir ce que l'avenir vous réserve, ne pas pouvoir prédire avec certitude le cours de la maladie et éprouver la crainte continuelle de mourir. La dynamique familiale s'en trouve alors bouleversée, profondément influencée par toutes les modifications de ses modèles de fonctionnement habituels (Habraken et al., 2008).

Par ailleurs, la médication nécessaire au traitement ou du moins à la stabilisation de la maladie, peut parfois induire des effets secondaires plus importants que les symptômes de la maladie elle-même, ce qui entraîne des inconforts et parfois même de la douleur physique et une souffrance psychologique (Ek, \& Ternestedt, 2008). Enfin, la qualité de vie du patient est altérée et il lui est habituellement fort difficile de maintenir l'ensemble des activités qu'il pratiquait auparavant (Habraken et al., 2008). Les personnes atteintes de maladies chroniques terminales représentent un pourcentage élevé des candidats à la transplantation. En plus de vivre la réalité d'avoir une maladie chronique en phase terminale, elles doivent faire face à celle de l'attente d'une transplantation. 


\section{Nécessité d'une transplantation}

Avant de débuter le processus d'attente, les médecins doivent d'abord évaluer si la greffe est indiquée chez le patient (Achille, 2002). Au Québec, l'admissibilité à la transplantation est évaluée dans les centres de greffes. Pour ce faire, une évaluation physique et psychologique est effectuée. Par la suite, lorsque le patient reçoit l'approbation de l'équipe de transplantation, il est inscrit sur la liste d'attente (Baran, 2008). Toujours au Québec, les greffes d'organes sont gérées par Transplant Québec, organisme ayant aussi pour mandat de promouvoir le don d'organe en sensibilisant la population ainsi que les professionnels de la santé à cette question. Transplant Québec élabore une liste unique de toutes les personnes en attente d'une transplantation d'organe au Québec (Transplant Québec, 2013a) et coordonne le processus du don d'organe, de l'identification du donneur au prélèvement (Transplant Québec, 2013a). Par contre, les médecins cuvrant dans les divers programmes de transplantation offerts dans les centres hospitaliers du Québec ont la responsabilité d'inscrire leurs patients sur la liste et de déterminer leur statut d'urgence. L'établissement a également la responsabilité d'aviser Transplant Québec s'il y a des modifications dans l'état de santé de la personne (Transplant Québec, 2013a).

Les critères d'attribution des organes, quant à eux, sont établis par un comité médical spécifique pour chaque organe et validés par un comité médical de Transplant Québec et par un comité d'éthique (Transplant Québec, 2013a). Ils sont généralement déterminés en fonction de la compatibilité anatomique, sanguine et tissulaire, du statut d'urgence et de la date d'inscription de la personne sur la liste d'attente de transplantation unique pour le Québec (Transplant Québec, 2013a). Le nombre de personnes en attente de transplantation 
augmente d'année en année, soit $11,52 \%$ de plus en 2012 , pour un total de 1250 personnes en attente, comparativement à 2007 (Transplant Québec, 2013c). Malheureusement, le nombre de donneurs ne suit pas la même courbe et tend parfois même à diminuer (Transplant Québec, 2013c). En 2012, 120 donneurs ont été recensés, soit vingt de moins qu'en 2007 (Transplant Québec, 2013c), ce qui pourrait expliquer que le temps d'attente ne cesse d'augmenter.

\section{L'expérience d'attendre une transplantation}

Une fois l'évaluation terminée et dès que l'aval de l'équipe de greffe est obtenu, la personne débute son attente. D'entrée de jeu, l'attente doit être explorée dans son sens large afin d'être en mesure d'avoir une compréhension plus globale de ce phénomène. À cette fin, l'analyse du concept de l'attente élaborée par Irvin (2001) selon le modèle d'analyse conceptuelle de Walker initie cette étape de la recension des écrits.

Cette analyse de concept a permis de faire ressortir la définition suivante :

L'attente est un phénomène à la fois statique et dynamique, couvrant une période imprécise de temps au cours de laquelle les manifestations d'incertitude concernant des résultats personnels sont suspendues, mais dans l'espoir d'atteindre un résultat escompté (Irvin, 2001, p. 128) [Traduction libre].

Elle a également fait émerger que les caractéristiques essentielles de l'attente sont l'incertitude et la perte de contrôle. L'incertitude qui découle de l'attente s'avère directement liée aux évènements incertains et à l'évolution de la maladie. L'incertitude provoquera du stress et le sentiment «d'être en suspens». De plus, les personnes en attente ressentent du stress parce qu'elles éprouvent le sentiment de ne pas avoir de contrôle sur la situation. Ce sentiment de perte de contrôle représente alors une menace pour la personne. 
Dans cette analyse, les individus tentent à la fois de gérer la détresse causée par l'évènement et de faire ce qu'ils peuvent pour éliminer la menace. Cependant, dans les cas d'incertitude et de perte de contrôle associées à l'attente, ces deux stratégies d'adaptation sont difficiles à utiliser. De plus, l'anxiété et le stress ressentis pendant la période d'attente sont susceptibles de s'intensifier lorsque la situation est considérée comme un état indéterminé, c'est-à-dire relié à un certain niveau d'incertitude (Irvin, 2001).

L'analyse du concept de l'attente élaborée par Irvin (2001) généralise sa définition à tous les types d'attente sans nécessairement s'attarder en profondeur à celle de la transplantation. Cependant, cette auteure fait référence à l'attente de la greffe en utilisant l'article de Jonsén et al. (2000) qui explore l'expérience de l'attente d'une transplantation du foie; ceux-ci précisent que la perception de l'attente se modifie selon certaines périodes. Ainsi, lorsque les patients ont identifié une amélioration de leur état de santé, ils ont une perception positive associée au temps d'attente (Irvin, 2001). Bien que les références soient disponibles, il aurait été intéressant de savoir combien d'articles portant effectivement sur l'attente ont été recensés lors de la rédaction de l'analyse de concept. Enfin, l'analyse est orientée par le modèle transactionnel de stress et de stratégies adaptatives de Lazarus et Folkman, ce qui peut sembler un peu limitatif.

Une fois l'attente définie au sens large par les propos d'Irvin (2001), les huit études recensées qui relatent l'expérience de l'attente dans le contexte précis de la transplantation peuvent maintenant être abordées (Bjork, \& Naden, 2008; Brown et al., 2006; Haugh, \& Salyer, 2007; Jonsén et al., 2000; Macdonald, 2006; Moran et al., 2011; Naef, \& Bournes, 2009; Sadala, \& Stolf, 2008). 
Tout d'abord, l'étude de Brown et al. (2006) explore la signification que les personnes atteintes d'une insuffisance hépatique donnent à l'expérience de l'attente d'une greffe. Il s'agit d'une étude qualitative réalisée selon l'approche phénoménologique. Les neuf entretiens non structurés ont été effectués auprès de six patients en attente d'une greffe de foie. Cette étude a fait émerger huit thèmes comme représentant l'expérience d'attendre une greffe de foie : 1) la transformation, 2) les médecins, les équipes de transplantation et la confiance, 3) de l'exaltation au désespoir, 4) la perte, 5) questionner le processus, 6) la recherche, 7) l'adaptation et 8) le paradoxe du temps.

Le premier thème, soit la transformation, fait référence au changement de perspective des participants en raison de la maladie terminale ainsi qu'au développement d'une nouvelle identité durant leur attente. Le second thème, les médecins, les équipes de transplantation et la confiance, expose les effets positifs et négatifs des relations entre les participants et les membres de l'équipe clinique. Il met également en évidence que les participants éprouvent le sentiment que la situation est sous le contrôle de l'équipe de greffe. Le troisième thème, de l'exaltation au désespoir, fait état du sentiment perpétuel d'être dans des montagnes russes, les hauts et les bas se calquant sur ceux de leur état de santé et sur les nouvelles, bonnes ou mauvaises, qui leur sont données. Le quatrième thème, la perte, rend compte des nombreux deuils que doivent faire les participants tout au long de leur attente (énergie, travail, identité, proximité des proches, etc.).

Pour sa part, le cinquième thème, questionner le processus, soulève que les participants continuent de s'interroger sur leur choix d'opter pour la greffe tout au long de l'attente, compte tenu de tous ses risques et de ses conséquences. Ils se questionnent également sur 
l'équité du processus. Quant au sixième thème, la recherche, il porte sur le fait que les participants dépensent beaucoup de temps et d'énergie à échafauder des théories sur leur expérience d'attendre une transplantation. Ils théorisent sur les effets de cette attente sur eux-mêmes, sur leurs relations avec les membres de l'équipe de greffe et sur son issue, à savoir s'ils seront greffés ou non. Le septième thème, l'adaptation, touche les moyens adoptés par les participants pour passer à travers cette période. Enfin, le huitième et dernier thème, le paradoxe du temps, souligne la préoccupation constante des participants à cet égard. En effet, le temps est vu comme s'étirant sans fin lorsqu'ils se concentrent sur les délais d'attente, mais aussi comme étant très court considérant qu'ils se préparent également à la possibilité de mourir bientôt (Brown et al., 2006).

Enfin, les participants de l'étude de Brown et al. (2006) décrivent la frustration qu'ils ont ressentie en lien avec le sentiment que le temps s'est arrêté. Ils rapportent une inquiétude constante que la greffe ne se produise jamais et soulignent aussi la conviction profonde qu'ils ont été oubliés par l'équipe de transplantation (Brown et al., 2006). L'attente est donc un «moment à part ». Ainsi, les participants éprouvent le sentiment d'être dans un purgatoire possédant son propre temps dont le cœur est représenté par la douleur, les routines cliniques, l'intrusion des téléavertisseurs et des fausses alarmes. Attendre sur la liste est donc reconnu comme un moment pour se préparer à la fois à la vie et à la mort.

L'espoir est ce qui permet aux participants d'attendre impatiemment, mais avec sérénité, bien qu'il leur soit difficile de faire face à l'isolement, à une profonde fatigue et à l'ennui (Brown et al., 2006). Afin de conserver cet espoir lors de l'attente, l'utilisation 
consciente du déni, de la pensée magique et de la pensée positive s'avère nécessaire. Enfin, malgré les sentiments initiaux de joie et de gratitude qui accompagnent le fait d'être placé sur la liste de transplantation, la dépression et l'instabilité émotionnelle sont communes à l'expérience de l'attente et découlent d'un sentiment profond de frustration (Brown et al., 2006).

Brown et al. (2006) ont respecté les critères de rigueur en matière de recherche qualitative de même que la philosophie d'Husserl dans la réalisation de leur étude. Les neuf participants étaient toujours en attente et la durée de celle-ci était variée. Les résultats rapportés ne font cependant pas état d'une redondance dans les données. Par ailleurs, l'analyse n'a pas été concomitante aux entrevues. Les deux étapes ont été réalisées de manière consécutive, ce qui a pu nuire à la richesse des propos recueillis lors des entrevues puisque certaines questions supplémentaires auraient pu émerger des analyses initiales. Enfin, comme l'étude a été réalisée aux États-Unis, la préoccupation des participants relative à l'équité du processus est liée au contexte dans lequel l'étude s'est déroulée.

La deuxième étude recensée est celle de Jonsén et al. (2000). Tout d'abord, le but de cette étude phénoménologique était de décrire l'expérience de l'attente d'une greffe du foie du point de vue des patients ayant une polyneuropathie familiale amyloïdotique. Un premier entretien, non structuré et ouvert, a été mené auprès de 14 patients nouvellement diagnostiqués. Un second entretien a été réalisé auprès de 11 d'entre eux, six entretiens un an après avoir été greffés et les cinq autres un an après le premier entretien. Des trois autres patients, deux étaient décédés et le dernier a refusé la deuxième rencontre. 
Selon les résultats de l'étude de Jonsén et al. (2000), l'attente est un processus continu dans le temps, impliquant non seulement les participants mais également le médecin et le système de soins de santé. Ce processus se divise en deux périodes précises : l'attente d'une décision et l'attente de la chirurgie pour lesquelles sept thèmes ont été identifiés : 1) marchander avec soi-même, 2) l'absence d'influence ou l'impuissance, 3) le soulagement et la joie, 4) l'impatience, 5) l'agonie, 6) le temps de se préparer et 7) le besoin d'information et de soutien. Ainsi, lors de l'attente d'une décision, le thème marchander avec soi-même réfère à la difficulté pour les participants de prendre la décision de tenter la greffe. Pour sa part, le thème absence d'influence ou impuissance met en évidence l'insécurité ressentie par les participants concernant leur éligibilité à la greffe lors de la période d'évaluation par l'équipe médicale. Durant l'attente de cette décision, les participants se demandent s'ils participent à un jeu injuste, sans règles précises.

Lorsque l'acceptation sur la liste de transplantation est obtenue, le processus se poursuit avec l'attente de la chirurgie (Jonsén et al., 2000). Ainsi, dans le thème le soulagement et la joie, ces sentiments prennent le pas sur le sentiment d'impuissance vécu par les participants lors de l'attente de la décision. À ce moment, l'attente devient plus positive puisque les participants ont maintenant devant eux une issue autre que la mort, bien que la date de la transplantation demeure toujours inconnue. De son côté, le thème, impatience, met en relief le paradoxe du temps vécu par les participants. Comme le temps passe rapidement alors que leur condition se détériore, ils se demandent s'ils en auront suffisamment pour se rendre à la transplantation. À l'inverse, le temps d'attente de la transplantation semble long et les participants se sentent impatients. Par ailleurs, attendre 
un traitement et voir progresser sa maladie évoquent, pour les participants, des sentiments qui sont communs avec l'agonie. Ce thème exprime, selon les auteurs (Jonsén et al., 2000), l'angoisse et l'incertitude qu'ils éprouvent quant à savoir s'ils vont survivre, tant à l'attente qu'à la chirurgie. Cette angoisse évoque également d'autres sentiments tels la peur de la mort, la résignation et l'angoisse de séparation.

Le sixième thème, le temps de se préparer, couvre les deux périodes ciblées par Jonsén et al. (2000), soit l'attente de la décision et l'attente de la chirurgie. Attendre une chose désirée est, certes, douloureux, mais permet également à celui qui attend de se préparer. Ainsi, le diagnostic d'une maladie terminale provoque une crise et place les participants dans l'attente d'une décision. Alors que des sentiments dépressifs sont exprimés et que même le suicide peut être envisagé, l'espoir d'être un candidat pour une transplantation et qu'elle soit une réussite ne s'estompe jamais. Pendant l'attente de la chirurgie, de nombreux participants s'efforcent d'être dans la meilleure condition physique ou mentale possible afin d'augmenter leurs chances de se rendre à la greffe, ce qui leur permet de garder courage et de rester positifs durant cette période. Des stratégies telles la pensée positive, l'autosuggestion ou le fait de penser à d'autres patients ayant survécu à leur transplantation les aident alors à garder cet état d'esprit (Jonsén et al., 2000).

Enfin, le dernier thème fait référence aux besoins d'information et de soutien lors de l'attente de la transplantation, et ce, tant lors de l'attente de la décision que pendant celle qui précède la chirurgie. En effet, les participants ont exprimé avoir eu peur d'être oubliés sur la liste et rapportent que les informations reçues n'étaient pas toujours suffisantes. Ils 
affirment avoir eu besoin d'un soutien professionnel puisqu'ils avaient de la difficulté à parler de leur peur de la mort avec leur famille (Jonsén et al., 2000).

Selon Jonsén et al. (2000), la phase où le patient attend la décision constitue une étape très difficile. Ce dernier doit donc utiliser de nombreuses stratégies pour parvenir à la traverser et réussir à garder espoir. Les participants de l'étude de Jonsén et al. (2000) ont tenté de maintenir leur espoir en se centrant sur les aspects positifs de la greffe et sur la signification de ce que pourrait représenter une vie en santé, une fois celle-ci réalisée. À partir du moment où ils ont été acceptés sur la liste d'attente, ils ont conservé cette attitude puisqu'ils avaient maintenant un but réel à atteindre. Dans cette étude, l'espoir s'avère primordial pour la survie des participants qui soulèvent que, sans l'espoir, il leur aurait été impossible de passer à travers l'attente (Jonsén et al., 2000).

Cette étude phénoménologique fait une description en profondeur des étapes de la méthode utilisée ainsi que des caractéristiques des participants. Ces détails permettent une meilleure transférabilité de l'étude et la redondance des données a été atteinte. Les verbatim utilisés dans les résultats reflètent bien chaque thème émergeant. L'analyse des données a été réalisée en six étapes, selon la méthode de Colaizzi. La septième étape, qui consiste à formuler un énoncé phénoménologique qui décrit le phénomène de manière aussi exhaustive que possible, n'a pas été réalisée ou n'est pas relatée dans l'article analysé. De plus, la validation qui sert à assurer une crédibilité à l'étude n'a pu être totalement effectuée puisque six entretiens de validation ont été réalisés un an après l'entretien initial. Ce long délai entre les deux rencontres peut avoir biaisé la validation. Enfin, trois des participants n'ont pu participer à la validation puisque deux d'entre eux 
sont décédés avant d'avoir pu le faire et que le dernier a refusé. Cependant, la description détaillée de chaque thème mène à une meilleure compréhension du phénomène.

La troisième étude présentée est celle de Macdonald (2006). Il s'agit d'une étude descriptive exploratoire qui avait pour but d'explorer de quelle manière les patients atteints de fibrose kystique et leur proche aidant vivent l'expérience d'attendre une transplantation et ce, en lien particulièrement avec les notions d'adaptation (coping) et de soutien. En ce qui concerne les participants recrutés, l'auteure a rencontré huit patients, quatre en prégreffe et quatre en postgreffe, et cinq proches. Elle a articulé ses résultats sur l'expérience de la transplantation autour de quatre thèmes, soit le déplacement, la confusion, la vie dans les limbes et le réajustement pour un bien-être, lesquels sont tous caractérisés par un sentiment d'incertitude. Enfin, chacun de ces thèmes correspond à l'une des étapes du processus de transplantation, c'est-à-dire lors de la référence à la transplantation, à l'occasion de l'évaluation pour l'acceptation sur la liste d'attente, pendant l'attente et après la transplantation (Macdonald, 2006). Seuls les thèmes des trois premières étapes seront rapportés ici, la période postgreffe ne faisant pas l'objet de la présente étude.

Le premier thème, le déplacement, réfère à une modification du concept de soi des participants lors de l'étape de la référence. Ainsi, l'annonce de la nécessité d'une greffe déplace le statu quo de la « vie normale » des participants. Macdonald (2006) soulève que, même si les patients savent que la greffe peut devenir une nécessité en raison de la détérioration de leur condition, ils ne sont généralement pas préparés à recevoir cette annonce, puisqu'ils n'ont pas conscience de la gravité de leur état. Alors que celle-ci 
génère de l'incrédulité et de la colère chez certains patients, les proches expriment plutôt des sentiments de joie et de soulagement, suggérant qu'ils sont davantage prêts à recevoir cette nouvelle que le patient lui-même.

Lors de la seconde étape, soit l'évaluation, la confusion apparaît comme le thème émergeant. Ainsi, malgré toutes les informations reçues afin de faciliter leur décision d'accepter ou de refuser la greffe, les patients vivent une énorme incertitude et éprouvent des émotions en dents de scie. À ce moment, les participants et leurs proches expriment le besoin de soutien, particulièrement celui de nature émotionnelle (Macdonald, 2006). Le troisième thème, soit la vie dans les limbes, correspond à la période d'attente qui dure jusqu'à ce que la transplantation ait lieu ou que la mort survienne. Comme Macdonald (2006) le fait remarquer, malgré leur condition précaire, les patients achètent du temps en prenant les mesures nécessaires pour maintenir leur état stable ou éviter une détérioration. Par ailleurs, ils craignent également de manquer le bateau, au sens où les faux appels font partie d'une réalité bien présente. En effet, les patients soulèvent que, lorsqu'ils doivent retourner à la maison sans leur organe, ils vivent une grande déception et davantage de stress, ce qui majore leur incertitude quant au fait qu'un autre donneur puisse être trouvé, et ce, avant qu'il ne soit trop tard. Ils ont donc peur d'être oubliés sur la liste d'attente (manquer le bateau) et craignent de déranger s'ils communiquent trop souvent avec leur centre de greffe pour savoir où ils en sont sur la liste (Macdonald, 2006).

Macdonald (2006) a utilisé un devis exploratoire descriptif puisqu'elle a basé ses questions d'entretien sur les grandes lignes de sa recension des écrits, ce qui s'avère pertinent. Toutefois, une approche phénoménologique, avec des questions plus larges et 
moins orientées aurait été intéressante afin d'explorer le vécu de ces personnes en attente de greffe. De plus, la catégorisation préalable de la période prégreffe en trois étapes paraît limitative, car certaines expériences sont alors reliées à une seule étape alors qu'elles sont sûrement présentes à tous les moments de l'expérience, comme le besoin de soutien par exemple. Par ailleurs, l'auteur fait mention de nombreux sous-thèmes mais plusieurs d'entre eux ne sont pas détaillés dans l'article, ce qui nuit à la compréhension et laisse le lecteur perplexe quant à certains éléments. Enfin, les thèmes ont été générés à partir tant des propos des patients que des proches, ce qui suggère que les personnes des deux groupes vivent la situation de l'attente de manière similaire, puisque les thèmes émergeants sont communs. Ce constat paraît toutefois paradoxal et mériterait d'être vérifié dans des études subséquentes.

Attendre sur la liste de transplantation est reconnu comme un chamboulement des objectifs personnels de tout individu (Bjork, \& Naden, 2008). La quatrième étude présentée, celle de Bjork et Naden (2008), propose une recherche qualitative descriptive visant à mieux comprendre le vécu d'attendre une transplantation pour 21 participants au stade terminal d'une maladie hépatique.

Les résultats des entretiens individuels semi-dirigés ont été regroupés sous quatre rubriques principales. La première porte sur les réactions psychologiques et l'expérience de recevoir le diagnostic et d'être placé sur la liste d'attente pour une greffe. Celle-ci est subdivisée en quatre sections, l'incertitude reliée à la vie et à la mort, la fatigue mentale reliée à la baisse d'énergie, la recherche profonde du sens de la vie et la sensation d'être fort mentalement. Suite à cette annonce, les participants vivent de profondes remises en 
question au plan existentiel. Plusieurs ont soulevé aussi que l'un des choix les plus difficiles qu'ils ont dû faire a été celui de réduire leurs activités physiques lors de l'attente. Seuls quelques-uns d'entre eux ont exprimé ne ressentir que peu ou pas de conséquences psychologiques relatives à leur situation (Bjork, \& Naden, 2008).

La seconde rubrique proposée est celle des inconforts physiques reliés à la maladie tels l'ascite, le prurit, l'œdème abdominal et celui des membres inférieurs. De son côté, la troisième rubrique concerne les expériences et les réactions reliées au fait d'être placé sur la liste d'attente. Ces dernières ont pour objet le choc, l'allégresse, le soulagement, la joie, le sentiment de ne pas avoir le choix, les pensées négatives et diverses craintes. Enfin, la dernière rubrique porte sur la vision qu'ont les participants de la chirurgie en soi et de la période post-opératoire. Ils rapportent notamment plusieurs craintes dont l'inconfort, la perte temporaire d'autonomie et d'intimité, celle que leur état se complique et, bien sûr, celle de mourir pendant ou suite à la chirurgie.

L'étude qualitative de Bjork et Naden (2008) utilise la théorie de l'incertitude de Mishel (1988) pour définir l'incertitude vécue par les patients en attente de transplantation. L'incertitude reliée à la vie et à la mort était un enjeu majeur de cette étude. Elle rapporte que neuf des 21 participants ont interprété le diagnostic de transplantation comme une opportunité (Bjork, \& Naden, 2008). Les 12 autres ont cependant évalué la situation en termes de danger. L'incertitude est perçue comme un danger par les futurs greffés puisque la peur de la mort et les évènements incertains tels que le moment de l'appel, le déroulement de la chirurgie et les risques de rejet sont perçus négativement. L'incertitude 
peut avoir des répercussions déplorables chez le patient comme la dépression, l'anxiété et la diminution de la condition physique (Bjork, \& Naden, 2008).

Cette étude a été réalisée auprès de 21 patients en attente de transplantation. Aucune information n'est fournie quant à l'atteinte de la redondance des données, ce à quoi il est plausible de s'attendre avec un échantillon de cette taille en recherche qualitative. De plus, dans l'article, les résultats sont traités largement. Les rubriques qui représentent des thèmes ne sont ni significatives, ni abordées en profondeur et certains éléments semblent mal classés ou chevauchent plus d'un thème. Enfin, la perception de l'attente qu'ont les participants peut être altérée par deux éléments importants du contexte de l'étude. Tout d'abord, les participants sont interrogés à l'hôpital, avant leur retour chez eux pour l'attente. Le temps entre l'annonce du pronostic de stade terminal de la maladie et l'entretien est donc relativement court. De plus, la transférabilité de l'étude demeure hautement questionnable puisqu'en Norvège, le temps d'attente avant d'être greffé est passablement bref, soit 24 jours en moyenne pour les participants de l'étude de Bjork et Naden (2008). Le fait que le temps d'attente soit court dans ce pays est sûrement connu des personnes en attente de greffe.

La cinquième étude présentée est celle de Moran et al. (2011). Cette recherche qualitative a été réalisée dans le but d'explorer l'expérience de patients atteints de maladie rénale terminale sous hémodialyse. Un échantillon de convenance de 16 participants a été recruté et deux entretiens ont été réalisés avec chacun d'entre eux. L'approche phénoménologique existentielle herméneutique de Heidegger a été utilisée et les données 
ont été analysées selon la méthode proposée par Diekelmann, Allen et Tanner (Moran et al., 2011).

L'élément général ou le point central qui chapeaute l'ensemble des trois thèmes qui émergent des propos des participants s'avère être l'expérience de l'attente d'une transplantation. Les thèmes suggérés sont vivre dans l'espérance, l'incertitude et être sur «pause».

Le premier thème fait ressortir que l'espoir occupe une place prépondérante lors de l'attente d'une greffe rénale. Celui-ci leur permet de passer à travers les jours, les semaines, les mois, voire les années jusqu'à ce qu'ils reçoivent leur transplantation (Moran et al., 2011). Le deuxième thème décrit l'expérience de l'incertitude pendant qu'ils attendent une greffe de rein. Les participants ont pris en considération le temps moyen d'attente pour une greffe rénale et ils s'y sont référés tout au long de l'attente. Cependant, dans bien des cas, ce délai s'est avéré plus long que la moyenne établie. À ce moment, l'espoir s'est transformé en incertitude de ne pas recevoir leur greffe. Enfin, être «sur pause» représente le dernier thème proposé. Conséquemment à leur expérience d'incertitude, les participants s'avèrent incapables d'envisager le futur. Ils sont limités par les contraintes reliées à l'hémodialyse et ne peuvent souvent plus travailler. Il en résulte la tristesse, la dépression et le désespoir. Leur vie se résume à attendre le téléphone ou la sonnerie du téléavertisseur. Tout le reste, ce qui constitue «la vraie vie», est en suspens (Moran et al., 2011).

Moran et al. (2011) suggèrent que la notion de supporter ou «d'endurer» développée par Morse dans son modèle de la souffrance caractérise certains traits des participants qui disent vivre sur «pause» en attendant la transplantation. En effet, les personnes suppriment 
leurs réactions émotionnelles et se concentrent sur le présent afin de passer à travers une situation qui leur semble insupportable. Selon ces auteurs, l'expérience d'attendre indéfiniment une greffe entraîne la suspension de ce que Heidegger nomme la temporalité originaire, qui se caractérise par de l'ennui profond (Moran et al., 2011).

Moran et al. (2011) fournissent une description détaillée de la méthode et des critères de rigueur auxquels ils se sont accolés afin de réaliser cette étude. Cependant, celle-ci n'avait pas pour but de mieux comprendre la réalité d'attendre une transplantation rénale, mais plutôt celui d'explorer le vécu des patients hémodialysés. Les résultats font ressortir une certaine compréhension du phénomène, mais les entretiens n'ont sans doute par permis d'approfondir ce que signifie être en attente d'une transplantation.

La sixième étude recensée, soit celle de Naef et Bournes (2009), visait à découvrir l'essence de l'expérience de l'attente et à générer de nouvelles connaissances sur la santé et la qualité de vie et ce, au moyen d'une approche phénoménologique herméneutique. À cette fin, des entretiens ont eu lieu avec 11 personnes qui étaient sur une liste d'attente pour une greffe de poumons (Naef, \& Bournes, 2009). Les résultats y sont discutés en relation avec la théorie de l'humaindevenant de Parse. Cependant, cette relation ne sera pas abordée ici, car elle nécessite une connaissance approfondie de cette théorie. Les principales conclusions de cette étude font ressortir trois thèmes principaux, à savoir : des contraintes pénibles (arduous constraints), l'anticipation du moment tant convoité de la transplantation (anticipating the cherished) et les engagements fortifiants (fortifying engagements) (Naef, \& Bournes, 2009). 
Dans le premier thème abordé, les contraintes pénibles, les participants décrivent l'attente comme une expérience très difficile, frustrante et contraignante, où le temps semble passer très lentement. De plus, les sentiments d'angoisse et de stress sont également mis à l'avant-plan. En outre, les participants ont comparé leur attente avec le sentiment d'être confiné : «comme étant ligoté et en prison». Ce sentiment d'emprisonnement s'explique par les nombreuses restrictions auxquelles ils doivent faire face lors de l'attente, en plus de devoir dépendre des autres (Naef, \& Bournes, 2009).

Le deuxième thème de l'étude de Naef et Bournes (2009), l'anticipation du moment tant convoité de la transplantation, fait ressortir comment les participent anticipent leur futur. En effet, les personnes en attente vivent dans l'espoir que la transplantation tant souhaitée se produise. Elles affirment avoir hâte que toute cette attente soit terminée et d'en finir avec ce processus. Certaines ont également affirmé qu'elles ne trouvaient pas d'autres solutions pour améliorer leur santé, car il n'existe pas d'alternative pour elles. Cependant, l'espoir de pouvoir être en mesure de reprendre une vie active les aide à passer à travers. L'incertitude face à ce que l'avenir leur réserve est également rapportée (Naef, \& Bournes, 2009).

Enfin, les engagements fortifiants constituent le troisième thème de cette étude. Il met en évidence comment l'attente permet aux participants de renforcer leurs liens avec Dieu, la famille, les amis ou les autres personnes attente. Les relations avec les autres s'avèrent édifiantes, encourageantes et génératrices d'espoir. Ils décrivent également comment ils se sentent à la fois proches et loin des autres. Par ailleurs, les participants indiquent avoir besoin d'être occupés avec des activités quotidiennes compte tenu de leur condition de 
santé. Bref, leurs relations sociales et leurs activités leur permettent de trouver la force et le courage de faire face à l'adversité de l'attente. (Naef, \& Bournes, 2009).

L'étude de Naef et Bournes (2009) approfondit la compréhension de l'expérience de l'attente. Cependant, l'utilisation de la théorie de Parse complexifie de beaucoup la compréhension des résultats et les rend peu accessibles aux professionnels qui ne sont pas familiers avec cette théorie. Toutefois, les recommandations pour la pratique infirmière peuvent être instaurées sans comprendre toute la profondeur de la théorie de Parse.

La septième étude consultée est celle de Haugh et Salyer (2007). Le but de cette étude qualitative, réalisée à l'aide d'un groupe de discussion, était d'explorer les perceptions de patients et des membres de leur famille concernant les interventions reçues pendant leur attente d'un cœur, l'efficacité perçue de ces interventions et les interventions non utilisées mais qui pourraient éventuellement être utiles (Haugh, \& Salyer, 2007). Le groupe de discussion a été utilisé comme méthode de collecte de données auprès de 11 participants, soit quatre patients greffés depuis moins d'un an, quatre patients en attente et trois membres de la famille (Haugh, \& Salyer, 2007).

Les thèmes qui ressortent de cette étude reflètent la perspective des patients et de leur famille concernant leur expérience d'attendre un donneur. Tolérer l'incertitude s'avère le thème principal décrivant la perception du temps d'attente. Pour leur part, les sous-thèmes représentent les interventions qui ont été considérées aidantes par les participants. Elles consistent à partager l'information, être sensible aux besoins de la famille, maintenir le respect et la dignité, faire de «petites choses supplémentaires » et faciliter l'adaptation (Haugh, \& Salyer, 2007). L'attente est marquée par l'incertitude et l'imprévisibilité. En ce 
sens, les participants mentionnent qu'ils doivent tolérer l'incertitude puisqu'ils savent que personne ne peut répondre à la question qui hante leurs pensées, soit : «Quand vais-je avoir un cour? » (Haugh, \& Salyer, 2007).

Par ailleurs, le partage d'information apparaît une intervention de choix durant l'expérience de l'attente. En effet, tous les participants ont souligné combien il était aidant pour eux de recevoir de l'information de la part des membres de l'équipe de soins. Toutefois, ils ont mentionné que cette intervention s'avère moins aidante si cette information n'est pas ajustée au rythme et à l'intérêt des personnes. Tous les patients et les membres de la famille ont également mentionné la sensibilité des intervenants aux besoins de la famille durant leur hospitalisation. Tous les participants ont aussi abordé leur besoin d'exercer un pouvoir sur la situation ainsi que le respect et la dignité qu'ils ressentent quand leurs opinions sont considérées dans les soins qui leur sont prodigués.

De plus, les participants ont mentionné que les «petites choses supplémentaires » effectuées par le personnel soignant leur permettaient de se sentir «normal» et de faire l'expérience de ce que plusieurs prennent pour acquis (permettre à l'un d'eux de recevoir la visite de ses chiens par exemple). Enfin, la spiritualité, l'humour et le soutien des autres patients sont reconnus par tous les participants comme des interventions aidantes qui facilitent l'adaptation durant l'attente d'un donneur (Haugh, \& Salyer, 2007).

Cette étude fournit des informations intéressantes concernant la présence de la famille dans le processus de la transplantation. Par contre, l'utilisation d'un groupe de discussion pour la collecte de données force à s'interroger sur la profondeur des entretiens réalisés. Des entretiens individuels auraient sans doute permis aux participants de s'exprimer plus 
librement et d'aller davantage en profondeur sur divers sujets. De même, certains participants ont pu être freinés de s'exprimer en présence de d'autres patients ou de la famille.

La huitième et dernière recherche recensée est l'étude phénoménologique de Sadala et Stolf (2008) qui avait pour but de mieux comprendre l'expérience d'être un greffé cardiaque. Celle-ci a été réalisée auprès de 26 patients au moyen d'un entretien individuel. Trois thèmes principaux ont émergé soit le temps d'attente vécu par le receveur d'un cœur, le donneur, la famille et les aidants naturels ainsi que la réflexion sur l'expérience vécue (Sadala, \& Stolf, 2008).

Bien que cette recherche concerne l'expérience d'être un greffé cardiaque, certains aspects abordent la période d'attente de la transplantation. Ainsi, le premier thème se concentre sur le temps vécu par les patients en tant que personne en phase terminale d'une insuffisance cardiaque et ce, à partir du moment où ils ont appris que leur état était critique. Les changements dans la façon de vivre leur vie quotidienne et les nombreuses limitations imposées par la maladie telles manger, boire, respirer et se déplacer ont marqué significativement leur vie et généré une grande souffrance. De plus, les participants prennent conscience de la finitude de leur vie. Ils constatent qu'ils vont vers la mort et tentent de conserver le peu de vie qu'il leur reste (Sadala, \& Stolf, 2008).

Les participants décrivent également le temps vécu dans l'attente comme une opposition au temps vécu dans l'activité. Leur vie est mise en suspens : ils ne font qu'attendre. La présence de l'angoisse liée à l'incertitude face à la vie ou à la mort se fait sentir constamment. La frustration fait également partie des sentiments vécus lors de 
l'attente puisque celle-ci est présente lors des faux appels pour la transplantation (Sadala, \& Stolf, 2008).

Enfin, le deuxième thème met en évidence les sentiments ambigus vécus par les participants durant tout le processus de transplantation incluant l'attente, à savoir l'espoir apporté par l'arrivée d'un donneur, mais aussi la culpabilité que la mort de quelqu'un d'autre puisse leur permettre de survivre. Ils soulignent enfin l'importance du soutien apporté par la famille et les professionnels de la santé (Sadala, \& Stolf, 2008).

Dans l'étude de Sadala et Stolf (2008), la taille de l'échantillon (26) est importante, mais il faut toutefois prendre en considération que le critère de sélection du temps écoulé depuis la greffe est très long, soit 14 ans (1990-2004). Ainsi, la moitié des participants (13) avaient vécu l'expérience il y a plus de cinq ans, dont neuf depuis 10 ans et plus, ce qui laisse place à l'interrogation quant à la précision des souvenirs rapportés. De plus, aucune allusion n'est faite à la redondance des données. Bien qu'en phénoménologie interprétative, la validation des thèmes auprès des participants n'apparaisse pas essentielle, elle aurait permis d'augmenter la crédibilité de l'étude. Toutefois, les résultats sont appuyés de verbatim significatifs.

En conclusion, les études recensées ont permis de mettre en lumière des aspects importants de l'expérience d'attendre une transplantation. L'espoir représente la force qui permet aux patients de passer à travers l'attente d'une transplantation (Brown et al., 2006; Jonsén et al., 2000; Macdonald, 2006; Moran et al., 2011; Sadala, \& Stolf, 2008). Plusieurs études soulèvent aussi que la condition de santé physique et mentale des participants se détériore constamment durant l'attente, en plus d'engendrer des limitations (Bjork, \& 
Naden, 2008; Macdonald, 2006; Sadala, \& Stolf, 2008). Certains d'entre eux rapportent même avoir le sentiment que leur vie est mise en suspens (Brown et al., 2006; Macdonald, 2006; Moran et al., 2011; Naef, \& Bournes, 2009; Sadala, \& Stolf, 2008). La perception du temps semble donc modifiée et celui-ci est qualifié d'infiniment long (Brown et al., 2006; Moran et al., 2011).

Bref, l'attente d'une transplantation est un processus éprouvant pour les participants (Naef, \& Bournes, 2009; Sadala, \& Stolf, 2008), d'autant plus qu'ils vivent de nombreuses frustrations et se sentent impuissants devant leur situation (Brown et al., 2006; Jonsén et al., 2000; Sadala, \& Stolf, 2008). Certains vont utiliser diverses stratégies pour passer à travers les épreuves de l'attente comme aller chercher de l'information supplémentaire, recourir à la pensée positive et se servir du déni conscient (Brown et al., 2006; Haugh, \& Salyer, 2007; Jonsén et al., 2000). Cependant, la majorité des études recensées font ressortir l'incertitude comme étant le concept central lié à l'attente d'une transplantation (Bjork, \& Naden, 2008; Brown et al., 2006; Haugh, \& Salyer, 2007; Jonsén et al., 2000; Macdonald, 2006; Moran et al., 2011; Naef, \& Bournes, 2009; Sadala, \& Stolf, 2008). C'est pourquoi il s'avère nécessaire d'explorer davantage le phénomène de l'incertitude au cours des lignes qui suivent. Afin d'y parvenir, la théorie de l'incertitude de Mishel $(1984,1988,1990)$ et le concept de l'incertitude de Penrod (2001a, 2001b, 2007) sont présentés succinctement.

\section{Incertitude}

Selon la théorie de Mishel (1984, 1988, 1990), l'incertitude se définit comme l'état cognitif (mécanisme de la pensée) créé lorsque la personne ne peut pas adéquatement structurer ou classer un évènement en raison d'un manque d'indices suffisants tels le 
résultat d'une biopsie, par exemple. Celle-ci est donc incapable d'assigner des valeurs précises à des objets et à des événements ou s'avère incapable d'en prédire les résultats avec précision. Cette théorie explique comment les personnes structurent cognitivement ces événements, de même que la signification qu'ils leur donnent.

Mishel (1984, 1988, 1990) identifie quatre objets principaux sur lesquels porte l'incertitude : 1) l'ambiguïté concernant l'état de la maladie, 2) la complexité des traitements et du système de santé, 3) le manque d'information sur le diagnostic et la gravité de la maladie, et 4) l'imprévisibilité de l'évolution de la maladie et de son pronostic.

La personne évalue son incertitude au moyen de deux processus distincts, soit l'inférence et l'illusion (Mishel, 1984, 1988, 1990). D'une part, l'inférence réfère à la personnalité, laquelle se construit à partir des croyances que la personne entretient par rapport à elle-même, de même que celles liées à sa relation avec les autres. Si le patient perçoit ses inférences positivement, l'incertitude sera évaluée comme une opportunité. Par contre, si elles sont envisagées comme menaçantes, l'incertitude sera alors évaluée comme un danger (Mishel, 1984, 1988, 1990). D'autre part, l'illusion se rapporte aux croyances favorables qui sont construites pour influencer positivement l'évaluation de l'incertitude (Mishel, 1984, 1988, 1990).

Selon Mishel (1990), lorsque l'incertitude est considérée comme un danger, un résultat néfaste est alors envisagé et la personne met en place des stratégies d'adaptation pour diminuer l'incertitude. À l'inverse, lorsque l'incertitude est perçue comme une opportunité, un résultat positif est attendu et des stratégies d'adaptation sont mobilisées pour maintenir l'incertitude. Une perception positive de l'incertitude, tels un traitement potentiel futur, une 
transplantation réalisable ou les bénéfices du recours aux médecines alternatives génère de nouvelles possibilités (Bailey Jr, Wallace, \& Mishel, 2007). Dans un cas ou dans l'autre, si les stratégies d'adaptation sont efficaces, la personne s'adaptera à la situation. Une adaptation difficile ne réfère donc pas à l'incertitude elle-même, mais à l'habileté de la personne à utiliser des stratégies d'adaptation efficaces dans la direction désirée.

La théorie de l'incertitude de Mishel $(1984,1988,1990)$ fournit aux professionnels des éléments importants pour jauger les impacts de l'incertitude vécue par le patient en attente, surtout si celui-ci lui attribue des inférences négatives. De plus, chaque personne possède un bagage différent qui influence sa vision de la maladie (Mishel, 1984, 1988, 1990) et les professionnels de la santé doivent être sensibilisés à cette réalité pour cibler judicieusement les interventions à faire auprès du patient (Bailey Jr et al., 2007).

Contrairement à Mishel, Penrod (2001a, 2001b, 2007), dans son analyse de concept, ne propose pas une vision probabiliste de l'incertitude. En d'autres termes, l'incertitude s'avère une expérience vécue par la personne et non une expérience qui est évaluée en termes de probabilités. Elle suggère plutôt que la connaissance précognitive, soit percevoir les événements sans un processus cognitif délibéré, apparaît importante pour déterminer l'incertitude (Penrod, 2007). Elle définit alors l'incertitude comme suit :

Un état dynamique dans lequel il y a un sentiment d'être incapable d'attribuer des probabilités à des résultats potentiels, qui occasionne une sensation désagréable et inconfortable, et qui peut être affectée (diminuée ou augmentée) par des réactions cognitives, émotives ou comportementales ou par le passage du temps ou des modifications dans la perception des circonstances qui l'ont provoquée. L'expérience de l'incertitude demeure omniprésente dans l'existence humaine et est modulée par des sentiments de confiance et de contrôle qui peuvent être hautement spécifiques à chaque personne (centrés sur l'événement) ou plus globaux (vision universelle) (Penrod, 2001b, p. 241). [Traduction libre] 
Pour Penrod (2001a, 2001b), l'incertitude réfère à une conclusion de doute fondée sur des éléments donnés et à un moment précis dans le temps. Elle soulève que le sentiment de confiance et le sentiment de contrôle sont des essences primaires qui déterminent la nature de l'expérience de l'incertitude. La confiance se définit comme la perception d'être en mesure de faire face à un événement incertain alors que le contrôle traduit la perception d'être capable d'avoir une influence sur les résultats (Penrod, 2001b). Penrod (2007) mentionne que les personnes utilisent des stratégies, soit des interventions ou des techniques pour influencer les sentiments de confiance et de contrôle perçus de la situation et, ultimement, celui d'incertitude. Enfin, la nature temporelle de l'incertitude est prédominante dans cette expérience. Elle est toujours vécue dans le présent, mais peut être influencée par les expériences passées et influencer les prévisions futures (Penrod, 2001a, 2007).

Pendrod (2007) évoque quatre types d'incertitude à partir des sentiments de contrôle et de confiance que la personne détient sur la situation. La Figure 1 schématise le concept de l'incertitude de Penrod (2007).

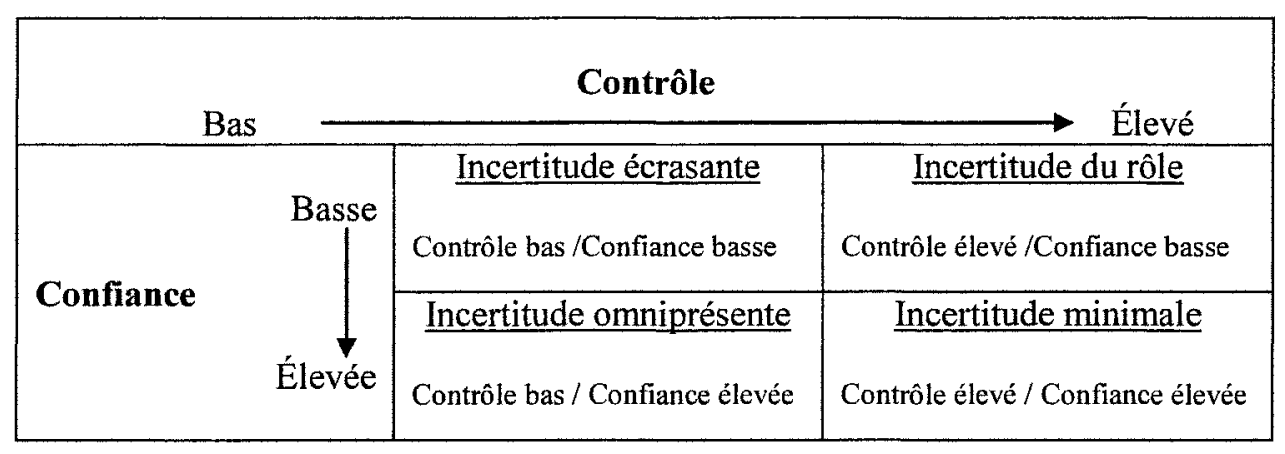

Figure 1. Types d'incertitude : équilibre des sentiments de confiance et de contrôle (Penrod, 2007, p. 661) [Traduction libre]. 
L'incertitude écrasante (overwhelming uncertainty) se manifeste par un sentiment minimal de confiance et de contrôle sur la situation. La personne a de la difficulté à intégrer cognitivement la situation et se sent paralysée par l'incertitude. En contraste, l'incertitude minimale (minimal uncertainty) est celle où la personne éprouve le sentiment de contrôler la situation et a confiance que celle-ci s'améliore. Dans le cas de l'incertitude omniprésente (pervasive uncertainty), la personne n'a pas le sentiment de contrôler la situation, mais reste confiante de trouver des moyens pour passer à travers. Finalement, l'incertitude reliée au rôle (role uncertainty) signifie que la personne a un bon contrôle sur la situation, mais qu'elle manque de confiance. La personne conserve un doute quant à sa capacité de prendre les bonnes décisions (Penrod, 2001a).

Penrod (2007) affirme que l'incertitude n'est pas un processus linéaire mais plutôt dynamique qui fluctue (types d'incertitude) selon la nature de l'événement (mode situationnel ou existentiel) et les sentiments de contrôle et de confiance perçus par la personne. Il s'agit donc d'un concept nuancé, qui offre plusieurs types d'incertitude selon la compréhension et l'interprétation vécues par la personne pour une même situation. Il est à noter qu'aucune étude recensée n'a eu recours à la théorie de l'incertitude de Penrod pour explorer l'incertitude vécue dans un contexte d'attente de transplantation.

Le chapitre suivant expose la méthode utilisée dans la présente étude afin de recueillir et d'analyser les propos recueillis auprès des participants. 


\title{
CHAPITRE III
}

\author{
MÉTHODE
}


Le troisième chapitre traite de la méthode de recherche qui a été utilisée pour réaliser cette étude. S'y succèdent donc la description du devis de recherche utilisé, du déroulement de l'étude, de la méthode de recrutement des participants, des outils et des étapes de la collecte des données, du plan d'analyse des données et des critères de rigueur en recherche qualitative auxquels répond cette étude. Enfin, les considérations éthiques sont abordées. Il importe toutefois de souligner au lecteur que le titre du mémoire a été modifié suite aux recommandations du jury lors du premier dépôt du document. La page titre n'est donc pas en tous points conforme avec ce qui est inscrit sur les documents produits en appendice.

\section{Devis}

Cette étude a été réalisée au moyen d'un devis qualitatif de type phénoménologique descriptif selon l'approche de Husserl. Edmund Husserl (1859-1938), philosophe allemand, est le fondateur de la phénoménologie transcendantale. Il cherche la solution dans le vécu, dans l'expérience qui sollicite la conscience personnelle (Giorgi, 1997). Sa philosophie représente la prise en compte de l'expérience vécue par les individus dans leur recherche de la vérité. La recherche phénoménologique vise à saisir l'essence du phénomène du point de vue de la personne qui le vit (Loiselle, Profetto-McGrath, Polit, \& Beck, 2007) et renvoie à la totalité des expériences vécues par cette dernière (Giorgi, 1997). Cette approche tente de décrire un phénomène tel qu'il a été vécu par la personne (Fortin, 2010).

À cette fin, quatre aspects majeurs de la philosophie Husserlienne doivent être pris en compte, soit 1) la conscience, 2) l'intentionnalité, 3) l'intuition et 4) la réduction phénoménologique (bracketing ou épochè et réduction eidétique) (Giorgi, 1997). Ainsi, pour Giorgi, la conscience représente le moyen pour accéder à tout ce qui se rapporte à 
l'expérience. Par expérience, Husserl entend l'intuition d'objets réels, soit ceux qui sont perçus dans le temps et dans l'espace mais également l'intuition des présences intangibles comme les hallucinations. L'intuition apparaît donc comme un terme plus large que celui d'expérience. De son côté, l'intentionnalité constitue la propriété essentielle de la conscience au sens où elle est toujours dirigée vers un objet. Par conséquent, l'objet ne prend un sens que dans sa relation avec la conscience du sujet (Giorgi, 1997).

Quant à la réduction phénoménologique, elle constitue la méthode philosophique pour faire ressortir l'essence d'un phénomène, soit le sens qui se maintient le plus durablement dans un contexte donné. Pour ce faire, le chercheur adoptera une attitude de bracketing ou épochè, laquelle consiste en la mise entre parenthèses des valeurs, croyances et connaissances du chercheur liées au phénomène étudié (Giorgi, 1997). Cette attitude vise à être entièrement présent au phénomène tel qu'il apparaît à la conscience. De son côté, la réduction eidétique consiste à déterminer le sens ou la nature universelle (essence) du phénomène au moyen de la méthode de «variation libre et imaginaire ». Pour ce faire, il faut faire ressortir l'essence du phénomène à l'aide des verbatim. Ainsi, l'imagination permet de varier de manière illimitée le phénomène en vue de dégager l'invariant eidétique ou le sens le plus constant du phénomène (Giorgi, 1997). La recherche phénoménologique s'avère particulièrement profitable lorsqu'un phénomène est méconnu ou incompris (Loiselle et al., 2007).

Dans cette optique, cette recherche phénoménologique a permis de décrire en profondeur le phénomène encore peu exploré d'attendre une transplantation. Cette description devrait permettre aux personnes qui vivent l'attente de se reconnaître et aux 
professionnels de la santé de mieux comprendre cette réalité et de développer des interventions visant à soutenir les patients lors de cette expérience.

\section{Déroulement de l'étude}

Cette section décrit le déroulement de l'étude. De manière plus spécifique, elle comporte la présentation du milieu et des participants à l'étude.

\section{Milieu}

Le milieu initial utilisé pour le recrutement des participants a été la Maison des greffés du Québec située à Montréal, sur la rue Sherbrooke. Cette maison a été mise sur pied en 1994 par Madame Lina Cyr, elle-même greffée du foie. Elle comprend 27 chambres et a pour mission d'accueillir des personnes en attente de transplantation ou en postgreffe et vivant à l'extérieur de Montréal. Elle leur fournit un endroit où elles peuvent demeurer à proximité de leur centre de greffe, à coût réduit et tout en bénéficiant d'un service de qualité (Cyr, 2010).

En 2011-2012, cette maison a accueilli 332 personnes en attente de transplantation ou ayant été greffées. De plus, les personnes résidant à la Maison des greffés sont en attente de greffe de divers organes tels le cœur, les poumons, le foie ou la moelle osseuse. Ce milieu a donc été choisi initialement parce qu'il donnait accès à des personnes qui ont une expérience approfondie du phénomène à l'étude.

Cependant, la contribution d'un second milieu a dû être sollicitée puisque le nombre de participants était trop faible, deux seulement ayant été recrutés à la Maison des greffés du Québec en près de deux mois. Au début du mois de novembre 2012, le président de 
l'Association régionale des greffés du Saguenay-Lac-Saint-Jean a donc été contacté. Cette association regroupe des patients de cette région qui vivent ou ont vécu l'attente d'une transplantation, tous types d'organes confondus. Elle a pour mandant de réunir des personnes greffées ou en attente afin de leur permettre d'échanger sur le sujet et offre aussi divers conseils à celles qui en éprouvent le besoin.

\section{Participants à l'étude}

L'échantillon cible pour cette recherche était constitué des patients en attente de transplantation ou ayant vécu l'attente d'une transplantation et vivant à l'extérieur de la ville où se situe le centre transplanteur et ce, peu importe le type d'organe attendu. Bien que la taille de l'échantillon recommandée en phénoménologie varie entre un et 20 (Fortin, 2010; Loiselle et al., 2007), l'échantillon utilisé pour cette étude a été constitué de sept personnes. En effet, après trois mois de recrutement, aucun autre participant ne s'est manifesté malgré les relances effectuées. La redondance a donc été partiellement atteinte puisque le recrutement a été cessé car personne d'autre n'avait manifesté son intérêt. Cependant, lors du dernier entretien, aucune nouvelle donnée n'a émergé.

Les participants ont été recrutés selon deux méthodes. L'échantillonnage par choix raisonné (ou intentionnel) a été initialement préconisé de manière à obtenir les informations nécessaires pour la compréhension du phénomène à l'étude (Loiselle et al., 2007). De plus, l'échantillonnage par réseaux (ou en boule de neige) a été privilégié comme seconde méthode, puisque l'étudiante chercheure a utilisé une personne greffée qui elle, est entrée en contact avec des participants potentiels qu'elle connaissait (Loiselle et al., 2007). Cette 
seconde méthode a été choisie considérant le fait que la première n'apportait pas les résultats escomptés quant au nombre de participants recrutés et que le temps filait.

L'étudiante chercheure a concentré son recrutement dans la ville de Montréal et dans la région du Saguenay-Lac-St-Jean puisqu'il s'agissait de recruter des participants ayant un vécu du phénomène à l'étude et non d'établir un échantillon représentatif de la population (Loiselle et al., 2007). Les critères d'inclusion étaient les suivants :

- Être âgé de 18 ans et plus;

- Être sur la liste d'attente de Transplant Québec pour une greffe d'organe (tous les organes confondus) ou avoir reçu une transplantation;

- Vivre à l'extérieur de la ville de son centre transplanteur;

- S'exprimer et comprendre le français.

Recrutement. Tout d'abord, madame Micheline Cyr Asselin, directrice de la Maison des greffés du Québec, a été interpellée. Une première approche téléphonique a été faite auprès de celle-ci pour lui expliquer le but de la recherche et obtenir son accord pour effectuer le recrutement des participants à la Maison des greffés. Par la suite, une lettre (Appendice A) lui a été acheminée le 15 mai 2012, afin de lui fournir une brève description de l'étude, les informations nécessaires pour entreprendre le recrutement et obtenir son autorisation pour procéder à la recherche dans son établissement. De plus, l'étudiante chercheure a communiqué par téléphone avec les membres des équipes de transplantation du Centre hospitalier universitaire de Montréal (CHUM) et de l'Institut de cardiologie de Montréal (ICM), pour les informer du projet de recherche et de valider auprès d'eux leur disponibilité en vue d'assurer au besoin un soutien psychologique aux patients recrutés. 
Par la suite, une lettre d'information sur le projet de recherche a été acheminée le 15 mai 2012 aux professionnels des programmes de transplantation concernés (Appendice B). Suite à la réception de l'autorisation pour le recrutement à la Maison des greffés du Québec, rédigée le 23 mai 2012 (Appendice C), et aux confirmations de fournir au besoin $\mathrm{du}$ soutien psychologique aux participants émanant des équipes de transplantation (Appendice D), l'approbation éthique du Comité d'éthique de la recherche de l'Université du Québec à Chicoutimi a été obtenue le 26 juin 2012 (Appendice E).

Afin de fournir une brève description de l'étude à la personne responsable de la Maison des greffés du Québec, cette dernière a été contactée par téléphone. De plus, la lettre d'information sur le projet de mémoire de maîtrise destinée aux professionnels des programmes de transplantation lui a été acheminée le 17 septembre 2012 (Appendice B). Aux fins de recrutement, une affiche (Appendice F) a également été placée bien en vue, dans une salle commune de la Maison des greffés du Québec et ce, du 20 septembre au 2 novembre 2012. Des appels téléphoniques ont été effectués à plusieurs reprises et des courriels ont été acheminés à la personne responsable du recrutement pendant cette période pour récupérer d'une part, les informations relatives aux personnes désirant participer à la recherche et, d'autre part, pour effectuer des relances auprès des participants potentiels.

Au début novembre 2012, considérant que seuls deux participants avaient été recrutés en presque deux mois et compte tenu des échéanciers académiques, l'Association régionale des greffés du Saguenay-Lac-Saint-Jean a été sollicitée pour le recrutement. Une première approche téléphonique a été faite auprès du responsable de l'association et, suite à son approbation, une lettre d'information sur le projet de maîtrise (Appendice G) a été remise 
par ce dernier aux membres de l'association lors d'un déjeuner. Par la suite, les personnes qui désiraient participer au projet ont logé un appel téléphonique à l'étudiante chercheure, ce qui a permis de recruter cinq nouveaux participants. La section suivante présente les outils de collecte de données.

\section{Outils de collecte de données}

L'étudiante chercheure a utilisé deux outils de collecte de données dans la présente recherche, soit un questionnaire sociodémographique (Appendice H) et un guide d'entretien (Appendice I).

\section{Questionnaire sociodémographique}

Afin de contribuer à documenter les caractéristiques des participants à l'étude, les données sociodémographiques portant sur la ville d'origine, le temps d'attente, le sexe, le groupe âge, le statut matrimonial et le nombre d'enfants ont été colligées sur un formulaire (Appendice H), au début de l'entretien.

\section{Guide d'entretien}

Pour faciliter la réalisation d'entretiens en profondeur, un guide d'entretien (Appendice I) composé de questions larges et ouvertes a été utilisé et ajusté tout au long de la collecte des données. Il a été validé au préalable par la directrice et la co-directrice de recherche puis a été l'objet d'un pré-test auprès d'une personne ayant vécu l'expérience d'attendre une transplantation mais ne participant pas à l'étude, afin de vérifier la clarté des questions. 
Les questions principales ont été élaborées en lien avec le but du projet qui visait à décrire et comprendre la signification de l'expérience vécue de patients en attente de transplantation vivant à l'extérieur des grands centres de la province de Québec. Des sousquestions ont été ajoutées au besoin, afin d'explorer plus en profondeur les données émergentes (Savoie-Zajc, 2003). La rétroaction a été utilisée afin d'encourager la personne participante à élaborer et à livrer le fond de sa pensée. La reformulation a également été utilisée comme autre technique pour améliorer les échanges (Savoie-Zajc, 2003). Les lignes qui suivent abordent les étapes de la collecte de données.

\section{Collecte de données}

La collecte des données s'est déroulée du 20 septembre 2012 au 15 décembre 2012. Au début de chaque entretien, d'une durée approximative d'une heure, l'étudiante chercheure est entrée en relation de manière chaleureuse avec la personne participante afin de la mettre à l'aise. Elle l'a rassurée sur la confidentialité des données recueillies tout comme sur le respect de l'anonymat et des règles en matière d'éthique de la recherche. Elle a également expliqué le déroulement de l'entretien, à savoir son implication et celle de la personne participante, précisé qu'il n'y avait pas de bonnes ou de mauvaises réponses et a répondu à ses questions. Enfin, le formulaire d'information et de consentement concernant la participation a été expliqué puis, les participants l'ont signé (Appendice J).

Les entretiens ont eu lieu à la Maison des greffés du Québec (2) à la chambre des participants pour assurer l'intimité, puisqu'aucun autre endroit privé n'était disponible, et à la résidence (5) des participants pour les personnes habitant au Saguenay-Lac-Saint-Jean. Les entretiens ont été menés à l'aide du guide d'entretien afin d'obtenir des données 
descriptives telles qu'exprimées par la personne participante. Tous les entretiens initiaux ont été enregistrés au moyen d'un enregistreur audio numérique afin que l'étudiante chercheure puisse se concentrer sur son interlocuteur et sur l'entretien en cours sans avoir à prendre de notes. À la fin de chaque entretien, elle a consigné dans son journal de bord toute note ou remarque en lien avec l'entretien (Fortin, 2010).

Une seconde rencontre a été nécessaire pour la validation de l'analyse qui a été effectuée afin de vérifier et d'approfondir certains aspects (Savoie-Zajc, 2003). La validation a eu lieu en avril 2013, suite à l'analyse des données. Seulement cinq des sept participants ont été en mesure de valider les unités de signification. En effet, lors de cette période, deux des participants étaient hospitalisés suite à des complications de leur état de santé. L'étudiante chercheure a donc pris la décision de ne pas valider l'analyse auprès d'eux puisque leur condition clinique ne leur permettait pas de le faire. Concernant les cinq autres participants, les rencontres pour la validation ont eu lieu à leur domicile. Elles n'ont pas été enregistrées et ont duré environ 30 minutes. Suite à la validation, aucune nouvelle information n'a été recueillie et aucun participant n'était en désaccord avec les unités de signification proposées. Cependant, les sous-thèmes n'ont pas été validés lors des entrevues de validation puisque l'étudiante chercheure n'avait pas arrêté définitivement son choix de sous-thèmes.

L'étudiante chercheure étant l'outil principal de collecte de données qualitatives (Fortin, 2010; Loiselle et al., 2007), elle a utilisé le bracketing, c'est-à-dire qu'elle a fait preuve d'ouverture et d'écoute empathique avec tous ses sens (Giorgi, 1997), en mettant ses connaissances personnelles, valeurs et à priori entre parenthèses (Loiselle et al., 2007). 
Pour ce faire, un journal de bord (Appendice K) a été complété par l'étudiante chercheure préalablement à la collecte de données et tout au long de l'étude, soit après chaque entretien et lors de l'analyse des données et de l'élaboration des sous-thèmes et des thèmes. Il contient tous les à priori, les valeurs, les croyances, les préjugés, les biais possibles, les connaissances théoriques, les observations, les commentaires, les pensées et les sentiments de l'étudiante chercheure en lien avec le sujet de l'étude (Giorgi, 1997; Loiselle et al., 2007). Enfin, il documente les renseignements recueillis et les pistes de décisions méthodologiques utilisées lors de la collecte et de l'analyse des données (Savoie-Zajc, 2003).

\section{Plan d'analyse des données}

Avant de procéder à l'analyse comme telle, les entretiens ont été retranscrits en s'accolant notamment aux règles suivantes, soit s'assurer de refléter la fidélité des propos des participants lors de la transcription, réaliser celle-ci dans un langage littéraire et épurer le matériel, c'est-à-dire retirer les patois et les informations qui pourraient permettre l'identification des participants (Savoie-Zajc, 2003). L'analyse a été faite de façon itérative à quelques reprises lors de la collecte des données, ce qui a permis de guider les entretiens subséquents. Certaines questions ont été modifiées dans leur formulation pour faciliter la compréhension du participant et lui permettre de livrer l'ensemble de son témoignage.

Par la suite, les sept étapes de la méthode de Colaizzi (1978) ont été utilisées pour analyser les données selon un processus permettant la recherche de l'essence de l'expérience humaine et individuelle de chacun. Les sept étapes peuvent se superposer afin 
d'assurer que le processus d'analyse correspond bien aux besoins spécifiques de l'étude (Colaizzi, 1978).

En premier lieu, une lecture initiale des verbatim transcrits a été réalisée pour avoir une idée générale des données recueillies. En deuxième lieu, les verbatim ont été examinés et les énoncés significatifs (ou unités de signification) de chacun des entretiens ont été extraits. Ensuite, chaque expression significative a été transformée en énoncé plus général afin d'éliminer certaines répétitions dans les propos des participantes.

En troisième lieu, le sens des énoncés significatifs a été formulé clairement. Il s'agit de reformuler l'énoncé général afin de faire émerger le sens qui découle des propos des participants et ainsi décrire l'expérience avec des expressions de sens qui représentent fidèlement leurs propos. Ensuite, il s'agit de regrouper certains éléments ayant un sens similaire afin d'éviter les répétitions.

En quatrième lieu, les significations émergeantes ont été regroupées en sous-thèmes puis en thèmes et des liens ont été établis entre les regroupements et les verbatim d'origine. Chaque thème retenu doit obligatoirement découler des expressions significatives afin d'être fidèle aux données brutes. Pour s'assurer que les différents thèmes élaborés représentent les données provenant des participants, il faut les comparer aux verbatim originaux.

En cinquième lieu, les résultats ont été intégrés à une description détaillée et approfondie du phénomène à l'étude. En sixième lieu, le phénomène de l'attente a été formulé clairement en une essence phénoménologique qui exprime ce que vit un adulte en attente de transplantation. Enfin, en septième et dernier lieu, les résultats ont été validés 
auprès de cinq des sept participants à ce projet, puisque deux des participants étaient hospitalisés et n'ont pu participer à la validation (Colaizzi, 1978; Loiselle et al., 2007).

Le logiciel NVivo 10 a été utilisé afin de consigner les pistes de décision de même que les sous-thèmes et les thèmes émergeant des propos des participants. De plus, des tableauxsynthèses ont été élaborés tout au long de l'analyse, afin de faciliter la compréhension des résultats analysés et de saisir certains liens au premier coup d'œil (Giorgi, 1997). La section suivante expose les critères de rigueur auxquels doit répondre une analyse qualitative.

\section{Critères de rigueur en analyse qualitative}

Afin d'assurer la rigueur des résultats, la démarche de recherche qualitative repose sur les critères suivants: la crédibilité, la fiabilité, la transférabilité et la confirmabilité (Loiselle et al., 2007).

\section{Crédibilité}

Selon Fortin (2010), la crédibilité se définit comme l'exactitude de la description faite par le chercheur au sujet de l'expérience vécue par les participants. Les résultats sont crédibles lorsqu'ils correspondent bien à la réalité vécue par d'autres patients qui vivent la même expérience mais qui ne sont pas des participants. Pour s'en assurer, l'étudiante chercheure a enregistré les premiers entretiens et les a fidèlement transcrits. Elle a également adopté une attitude de bracketing tout au long du processus de recherche (Giorgi, 1997). Ayant vécu elle-même l'expérience de l'attente d'une transplantation puisqu'un membre de sa famille immédiate a été greffé, l'utilisation d'un journal de bord s'est avérée d'autant plus indiquée. 
La rédaction continue du journal de bord lui a permis de reconnaitre son vécu et ses connaissances personnelles sur le sujet. En effet, l'étudiante chercheure avait beaucoup d'idées préconçues concernant les résultats qui allaient émerger des entretiens, compte tenu de sa situation d'être une proche de personne ayant été greffée. Ainsi, elle a été à même de constater que de nombreux éléments issus de la collecte et de l'analyse des données ne correspondaient pas aux idées préconçues qu'elle avait mises de côté et consignées dans son journal. De plus, cette rigueur dans la tenue du journal de bord lui a permis de reconnaitre les sentiments générés chez elle lors des entretiens, lesquels auraient pu influencer les propos des participants si elle ne les pas avait notés. À la fin des entretiens de validation, quelques participants ont demandé à l'étudiante chercheure les raisons qui avaient motivé le choix de son sujet de recherche. À ce moment seulement, elle a révélé aux participants qui en faisaient la demande la nature de ce choix puisqu'elle ne pouvait plus influencer le discours de ceux-ci.

Afin d'obtenir une vision plus complète et plus contextuelle du phénomène à l'étude, la triangulation des chercheurs (peer debriefing) a également été utilisée (Loiselle et al., 2007). L'analyse et l'interprétation des données ont été réalisées par l'étudiante chercheure ainsi que par la directrice et la co-directrice du projet de mémoire. Enfin, un deuxième entretien auprès des participants de l'étude a permis de vérifier leur adhésion à la description de leur réalité vécue (Loiselle et al., 2007). 


\section{Fiabilité}

La fiabilité est un critère qui renvoie à l'authenticité des données (Loiselle et al., 2007).

Pour évaluer celle-ci, la technique de reproduction progressive a été utilisée, c'est-à-dire que l'étudiante chercheure, la directrice et la co-directrice ont traité les données séparément pour les comparer par la suite (Loiselle et al., 2007). De plus, l'étudiante chercheure a rédigé dans son journal de bord des notes lui permettant d'expliquer les décisions qui ont été prises tout au long de la recherche (tracé de l'audit) (Fortin, 2010).

\section{Transférabilité}

Le critère de transférabilité indique si les conclusions tirées à partir des données recueillies peuvent s'appliquer dans d'autres contextes (Loiselle et al., 2007). Pour ce faire, chacune des étapes de la recherche a été décrite minutieusement et tout le matériel utilisé pour la collecte des données est demeuré accessible, facilitant ainsi la vérification du processus de recherche. L'étudiante chercheure a fourni également une description étoffée (thick description) du contexte de l'étude, à savoir les caractéristiques des participants, les milieux et les moments où la recherche s'est déroulée (Fortin, 2010). De plus, l'utilisation du logiciel NVivo 10 permet d'identifier le processus d'analyse effectué, ce qui pourrait faciliter la transférabilité de l'étude.

\section{Confirmabilité}

La confirmabilité évalue la qualité de la recherche en s'assurant que les résultats représentent bien la réalité vécue par les participants et non le point de vue du chercheur (Loiselle et al., 2007). Pour ce faire, le bracketing utilisé tout au long de la collecte et de 
l'analyse des données contribue à assurer l'objectivité des données (Fortin, 2010). De même, la validation des résultats auprès des participants vise le même objectif. L'accès direct aux données à des fins de vérification, soit la transcription des entretiens, le journal de bord et les notes de terrain a également permis d'assurer la véracité des données (Loiselle et al., 2007). La description en profondeur, tant du vécu des personnes participantes que de chacune des étapes de la recherche, a facilité la démonstration des pistes de décision qui ont conduit l'étudiante chercheure aux résultats présentés. De plus, les résultats de l'analyse sont appuyés par des verbatim significatifs (Loiselle et al., 2007).

\section{Considérations éthiques}

Ce projet de recherche a été approuvé par le Comité d'éthique de la recherche de l'Université du Québec à Chicoutimi (Appendice E), puisqu'il a été réalisé dans le cadre d'un programme de deuxième cycle en sciences infirmières dans cette université. Afin que cette recherche respecte les règles éthiques relatives à la recherche sur des êtres humains et compte tenu de la nature potentiellement émotive des propos des participants, l'accès à un suivi psychologique a été mis sur pied. Chaque équipe de transplantation a accepté de mettre à la disponibilité des participants le psychologue qui y était rattaché. Ainsi, le participant qui éprouvait le besoin d'obtenir un suivi psychologique n'avait donc qu'à communiquer avec son équipe de transplantation pour en bénéficier.

De plus, suite à la difficulté de recrutement à la Maison des greffés du Québec, une seconde méthode de recrutement a été établie et les critères d'inclusion ont également dû être modifiés. Au critère vivre l'attente d'une transplantation s'est ajouté ceci : avoir 
attendu une transplantation. Cette modification a été entérinée par le Comité d'éthique de la recherche de l'UQAC le 15 novembre 2012 (Appendice L).

Afin de s'assurer et de respecter la confidentialité des participants, un pseudonyme a été attribué à chacun et une liste de correspondance des noms et des codes a été rédigée puis placée sous clé. De plus, une attention particulière a été portée afin de retirer les extraits d'entretien pouvant permettre l'identification des participants. Le matériel (questionnaires des données sociodémographiques, retranscriptions des verbatim, enregistrements) est entreposé sous clé, au module des Sciences infirmières et de la santé de l'Université du Québec à Chicoutimi, pour une période de sept ans, sous la responsabilité de l'étudiante chercheure.

Le chapitre suivant présente les résultats de la recherche de même que les sous-thèmes et les thèmes qui émergent de leur analyse. 
CHAPITRE IV

RÉSULTATS 
Le quatrième chapitre expose les résultats de cette recherche de type phénoménologique descriptive selon l'approche phénoménologique transcendantale de Husserl (Giorgi, 1997). Tout d'abord, les données sociodémographiques des participants de l'étude sont décrites. Par la suite, les sous-thèmes et les thèmes en lien avec la question de recherche sont détaillés afin de tenter de brosser une description de l'expérience de vivre l'attente d'une transplantation, à la lumière des entretiens des participants.

\section{Données sociodémographiques et cliniques des participants}

Aux fins de cette étude, le recrutement a été effectué à la Maison des greffés du Québec et auprès de l'Association régionale des greffés du Saguenay-Lac-Saint-Jean. Ainsi, sept participants, soit six hommes et une femme, ont accepté d'y prendre part. Tel que mentionné précédemment et afin de préserver l'anonymat de ceux-ci, l'étudiante chercheure a utilisé des pseudonymes pour chacun d'eux. Il importe de souligner que cinq des participants avaient reçu leur transplantation au moment de la collecte des données et que deux étaient toujours en attente. Les premiers racontaient donc des souvenirs de la période de l'attente alors que les deux autres vivaient l'attente au quotidien lors des entretiens. Des cinq participants transplantés, un l'était depuis moins de deux mois.

Concernant leur état civil, cinq des participants étaient en couple lors de l'attente. De plus, cinq d'entre eux avaient des enfants d'âge adulte et un participant avait des enfants en bas âge lors de l'attente de la transplantation. Quant au temps d'attente, trois des participants avaient attendu entre un et trois mois alors que quatre d'entre eux avaient attendu 13 mois et plus. Pour le type d'organe à recevoir, trois des participants ont vécu l'attente d'une transplantation cardiaque, trois celle d'une transplantation pulmonaire et un 
participant a vécu l'attente d'une transplantation rénale. En ce qui concerne leur âge, quatre participants avaient entre 50 et 59 ans, l'un d'entre eux avait entre 40 et 50 ans et les deux autres avaient 60 ans et plus lors de leur attente. Les données sociodémographiques des participants sont présentées au Tableau 1.

Tableau 1

Données sociodémographiques des participants

\begin{tabular}{|c|c|c|}
\hline Caractéristiques & $\begin{array}{l}\text { Nombre de } \\
\text { participants }\end{array}$ & Pourcentage \\
\hline \multicolumn{3}{|l|}{ Sexe } \\
\hline Femme & 1 & 14,28 \\
\hline Homme & 6 & 85,71 \\
\hline \multicolumn{3}{|l|}{ Âge (ans) } \\
\hline \multicolumn{3}{|l|}{$18-29$} \\
\hline \multicolumn{3}{|l|}{ 30-39 } \\
\hline $40-49$ & 1 & 14,28 \\
\hline $50-59$ & 4 & 57,14 \\
\hline 60 et plus & 2 & 28,57 \\
\hline \multicolumn{3}{|l|}{ État civil } \\
\hline Marié /conjoint de fait & 5 & 71,42 \\
\hline Célibataire & 1 & 14,28 \\
\hline Divorcé ou séparé & 1 & 14,28 \\
\hline \multicolumn{3}{|l|}{ Veuf } \\
\hline Sans enfant & 1 & 14,28 \\
\hline \multicolumn{3}{|l|}{ Avec enfants } \\
\hline D'âge adulte & 5 & 71,42 \\
\hline En bas âge & 1 & 14,28 \\
\hline \multicolumn{3}{|l|}{ Temps d'attente (mois) } \\
\hline $1-3$ & 3 & 42,85 \\
\hline \multicolumn{3}{|l|}{ 4-6 } \\
\hline \multicolumn{3}{|l|}{$7-9$} \\
\hline \multicolumn{3}{|l|}{$10-12$} \\
\hline 13 et plus & 4 & 57,14 \\
\hline \multicolumn{3}{|l|}{ Statut d'attente } \\
\hline Greffé & 5 & 71,42 \\
\hline En attente & 2 & 28,57 \\
\hline
\end{tabular}




\section{L'expérience d'attendre une transplantation}

Le but de ce projet de recherche était de décrire et comprendre la signification de l'expérience vécue de patients en attente de transplantation vivant à l'extérieur des grands centres de la province de Québec. L'analyse approfondie des données recueillies à partir des entretiens effectués lors de la collecte de données et réalisée selon la méthode de Colaizzi (Loiselle et al., 2007) a permis de faire ressortir l'expérience du vécu des patients en attente de transplantation. Des sous-thèmes ont été développés à partir des verbatim rapportés et, de ces sous-thèmes, trois thèmes ont émergé, soit : la volonté de vivre, garder l'espoir de vivre malgré la tourmente de l'incertitude et mettre sa vie en suspens. Ils sont illustrés à la Figure 2. Afin d'assurer une meilleure compréhension de la signification de l'attente d'une transplantation, des extraits des verbatim issus des propos des participants sont rapportés tout au long de ce chapitre. 


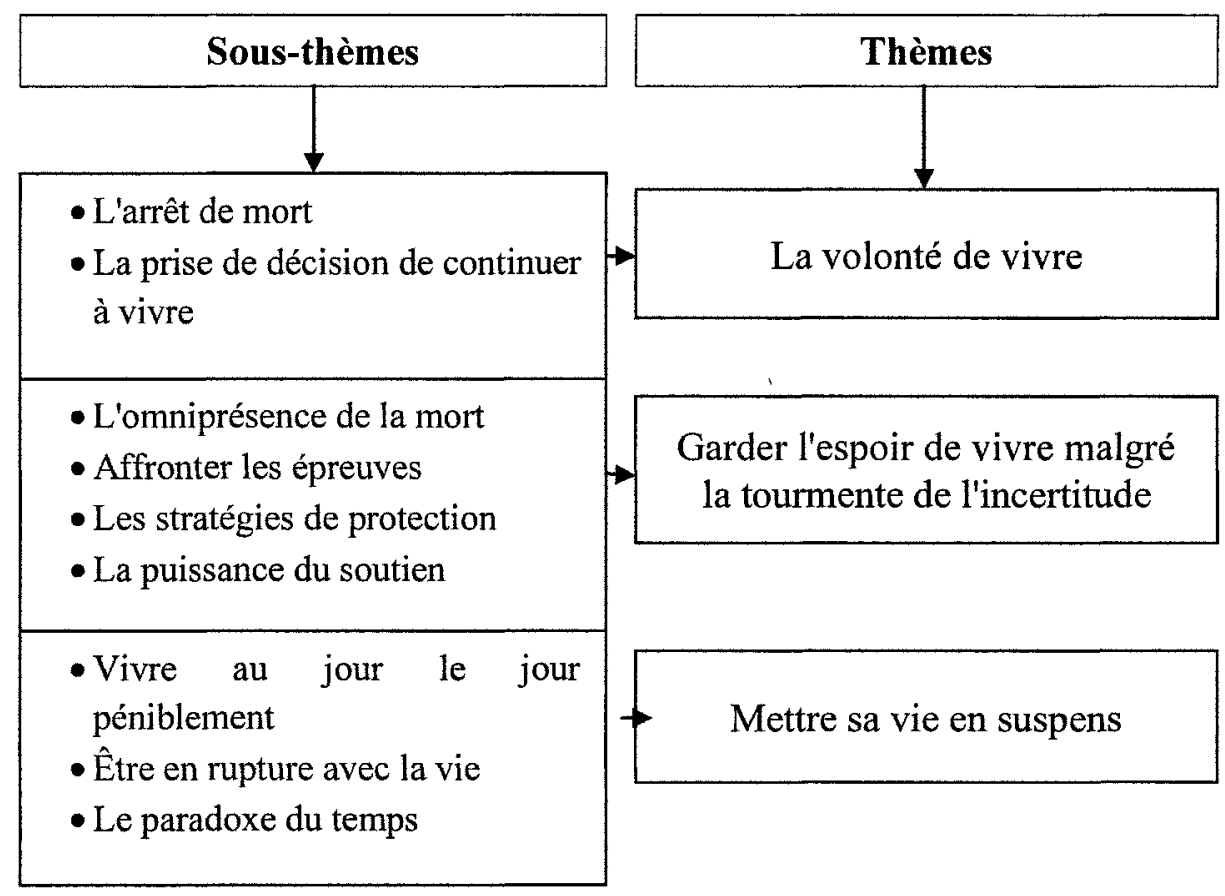

Figure 2. Sous-thèmes et thèmes de l'expérience d'attendre une transplantation.

\section{La volonté de vivre}

Vivre l'attente d'une transplantation s'avère une période difficile et remplie d'embûches, tant pour les participants que pour leurs proches. Cependant, avant de se retrouver sur la liste d'attente, les participants doivent souvent faire face à une maladie chronique terminale qui les mènerait sans aucun doute à la mort si la possibilité de transplantation ne leur était pas offerte. Le désir de vivre constitue un point culminant de l'expérience d'attendre une transplantation. C'est cette volonté de vivre, profondément enracinée dans chacun d'eux, qui permet aux participants de faire face aux épreuves auxquelles ils sont confrontés tout au long de l'attente.

Bien que les participants atteints d'une maladie terminale conçoivent la gravité de leur état, l'annonce de la nécessité d'une transplantation pour continuer à vivre constitue, selon 
eux, un choc important. L'annonce de l'imminence de la mort, la difficulté d'intégrer cognitivement la situation et la prise de décision d'opter ou non pour la transplantation demeurent des éléments qui influencent la manière dont se vit l'attente. Cet arrêt de mort et la prise de décision de continuer à vivre sont déterminants dans la signification que les participants donnent à leur expérience. Pour passer à travers l'attente, la volonté de vivre doit grandement dépasser la crainte des embûches qui jalonnent le chemin jusqu'à la greffe. Cette détermination est bien présente chez Pierre qui désire vivre plus que tout pour le bien de ses enfants :

C'est là que je vois que la famille est excessivement importante. Tous les jours, je voyais (nom de son fils) qui avait trois ans et je me détournais pour ne pas pleurer et je me disais: "Il faut que je vive, il vaut que je vive, il faut que je vive »... Tu aimes tes enfants.

Cette volonté de vivre est explicitée de manière plus approfondie au moyen de deux sous-thèmes soit : l'arrêt de mort et la prise de décision de continuer à vivre. Dans le premier sous-thème, l'arrêt de mort, les participants décrivent ce qu'ils ont vécu lors de l'annonce de la nécessité d'une transplantation. Pour la plupart d'entre eux, l'annonce de la transplantation n'a pas été le plus grand choc. En effet, recevoir un pronostic de mort à court terme et parfois même de mort imminente leur cause un choc encore plus important. Le verbatim qui suit représente bien le sentiment d'arrêt de mort que Paul a ressenti lors de l'annonce du pronostic de sa maladie :

C'est quand on t'annonce qu'il n'y a plus rien à faire avec toi. Tu es condamné. Ils disent qu'il y aurait peut-être juste une chose qui pourrait me sauver et c'est la greffe. C'est là que ça fait mal. C'est le diagnostic qui fait mal. Après ça, tu essaies de trouver la manière d'arranger ça, mais la seule manière, c'est que ça ne se soigne pas. Tu ne peux pas rien faire avec ça. Ça te prend une greffe absolument. 
De plus, pour Paul, recevoir son pronostic est encore plus difficile en raison de la manière dont il l'a appris. En effet, le sentiment d'être ignoré et de n'être qu'un numéro contribue à la difficulté de prendre conscience de la sévérité de sa condition :

Tu es en petite jaquette dans un coin, il fait noir comme dans une garde-robe et les deux médecins... Elle ne comprend plus rien, elle appelle l'autre médecin : "Viens voir ça, ça pas de bon sens". Tu ne le sais pas ce que tu as. Tu penses que tu as pogné une pneumonie, tu ne sais pas. Ils ne sont pas conscients que tu es là. C'est comme s'ils marchaient avec un numéro, toi tu es dans le coin, sur la petite chaise et eux sont tous les deux là et regardent la machine, sur l'écran: "Hein ça ne se peut pas, il est supposé être mort ça fait 10 ans, comment ça qu'il respire encore, c'est impossible, il est mort ». C'est comme ça qu'ils t'annoncent que tu es perte totale.

La condition clinique préalable à l'annonce du pronostic influence également le choc de cette annonce. Ainsi la majorité des participants ont vécu une longue trajectoire de maladie chronique avant de recevoir ce pronostic, sauf pour l'un d'eux. Éric a contracté une infection virale qui a détruit son cœur en quelques jours. Suite à des complications, il a émergé du coma une semaine après son hospitalisation, sous assistance d'un cœur mécanique. C'est à ce moment qu'il a compris qu'il devait recevoir une transplantation. La perte de l'organe malade a été pour lui un moment difficile, à savoir le deuil d'un cœur qui l'avait toujours bien servi jusque-là. Une grande vague de tristesse l'a envahi lorsqu'il a évoqué cette période de sa vie : «Ç’a été assez spécial parce que là, je savais que j'allais aller vers la greffe. Ils ont essayé de sauver mon cour au début et ils n'ont pas été capables » (pleure, se lève pour aller chercher un papier mouchoir).

Comme le soulignent ces témoignages, l'annonce du pronostic terminal d'une maladie, que celle-ci soit relativement subite ou chronique, s'avère un choc important pour la personne. L'ampleur de ce choc est également influencée par la manière dont l'annonce est 
faite et par l'état de santé de la personne. Recevoir le diagnostic de mort imminente représente une expérience extrêmement pénible pour la personne qui doit faire face à cette éventualité.

Cependant, l'annonce de la possibilité d'une transplantation constitue pour les participants une possibilité de prolonger leur vie et ils doivent dès lors prendre en considération cette nouvelle avenue qui s'offre à eux. Bien que pour certains, il s'agisse d'une décision facile à prendre, pour d'autres, cette prise de décision s'avère plus complexe. De nombreux éléments tels le désir de vivre, une délivrance face à la maladie, le sentiment de liberté et la difficulté à intégrer la maladie au quotidien influencent la prise de décision de continuer à vivre. Chez certains d'entre eux, la possibilité d'une transplantation provoque un profond soulagement et représente l'espoir d'une vie meilleure, quand ce n'est pas l'espoir de vivre tout court. La prise de décision de tenter la greffe constitue alors une option profondément enracinée dans ce désir de vivre, comme le souligne Paul :

Ils disent : "Tu vas mourir ou il te reste juste ça. Mourir ou la greffe. Ça te prend une greffe absolument". Donc là, tu décides si oui ou non tu veux la faire. L'envie de vivre, c'est juste ça.

La possibilité de transplantation signifie pour Pierre une délivrance face à la maladie avec laquelle il jongle depuis plusieurs années : «Ç’a été une délivrance. Moi c'est vrai que dans mon cas, je parle de ça avec les autres greffés. Moi, je l'attendais, la greffe». Cette envie de vivre constitue donc le moteur de la décision d'entreprendre le processus de transplantation, et ce, malgré les complications qui risquent de se produire tout au long de l'attente et les risques réels reliés au fait de recevoir une transplantation. Daniel, qui 
comprenait bien les risques de complications explique que, malgré tout, la possibilité de vivre quelques années de plus demeure plus importante que les risques encourus : « $J^{\prime} a i$ compris que c'est des affaires qui peuvent arriver, mais pas au point de m'arrêter, non. Non, j'aimais trop respirer. S'il y avait possibilité de greffe, que tu peux vivre une couple d'années de plus c'est sûr que...».

Pour Mario, la greffe représente une possibilité de liberté. En effet, sous dialyse péritonéale depuis plusieurs années, il entrevoit l'éventualité d'une amélioration de sa qualité de vie :

J'avais hâte à la transplantation pour la liberté. Je savais que mon état de santé allait s'améliorer beaucoup. Je savais que je n'aurais plus besoin de la dialyse pour continuer à vivre. Ça t'apporte beaucoup de liberté, c'est ce que je voyais dans la transplantation.

Par contre, choisir de continuer à vivre fait ressortir la difficulté de la prise de décision concernant la transplantation. Si elle est facile pour certains, elle s'avère beaucoup plus difficile pour d'autres. Ainsi, cette étape a été beaucoup plus pénible pour Éric et Hélène. Ayant tous deux de la difficulté à intégrer la maladie à leur quotidien, l'éventualité d'une transplantation a été vécue douloureusement. En effet, l'intégration cognitive de la maladie à sa vie influence directement la prise de décision de s'engager ou non dans le processus de transplantation. Ainsi, Hélène a refusé la transplantation une première fois parce qu'elle croyait que son état de santé allait s'améliorer :

J'ai refusé la première fois, j'ai refusé complètement. De toute façon, ça m'a pris du temps à accepter le fait que je sois malade comme ça. Je ne me rendais pas compte que j'étais aussi malade. Je me disais : "Haaa... ça va passer, ça va revenir ". Non, j'ai refusé quand le médecin m'avait offert ça. J'ai refusé. 
C'est lors d'une détérioration de sa condition clinique qui l'a menée près de la mort qu'elle a pris la décision de vivre le processus de la transplantation. Quant à Éric, l'obligation d'accepter l'éventualité de la mort de quelqu'un d'autre en échange de sa propre vie a rendu d'autant plus difficile la prise de décision d'entreprendre ce processus :

Après ça, quand ils m'ont dit que j'allais aller à la greffe, c'était plus dur, dans ma tête, c'était spécial. Comment je te dirais ça. Vivre tout ça, de savoir que quelqu'un allait mourir pour me sauver. J'ai eu de la misère à accepter. C'a été assez spécial.

En résumé, le thème la volonté de vivre fait ressortir les premiers moments vécus par les participants pendant le long parcours de l'attente d'une transplantation. C'est au tout début de ce chemin parsemé d'embûches qu'ils doivent faire face à cette nouvelle réalité d'une mort imminente. Suite au choc de l'annonce du pronostic, repousser l'éventualité de la mort devient réalisable avec la transplantation, mais cette possibilité doit être bien évaluée à la lumière de tous les risques qui y sont inhérents. L'imminence de la mort facilite la prise de décision de tenter la greffe. La prépondérance de l'envie de vivre est bien présente dans les verbatim et constitue, dans certains cas, le moteur de la prise de décision d'y avoir recours. Prendre le risque de vivre ou de s'engouffrer vers une mort certaine, tel est le choix déchirant que les participants ont eu à faire.

\section{Garder l'espoir de vivre malgré la tourmente de l'incertitude}

Garder l'espoir de vivre malgré la tourmente de l'incertitude est le second thème de l'expérience d'attendre une transplantation. Au cœur de cette expérience, l'espoir constitue un aspect important. Il permet aux participants de faire face aux épreuves rencontrées tout au long de cette attente. Bien que personne ne soit certain de ce que sera demain, la route 
qui mène les personnes en attente vers la transplantation est sans contredit parsemée d'embûches, de craintes et d'incertitude sur ce que l'avenir leur réserve. L'espoir de vivre permet aux participants de faire face à cette adversité, comme en témoigne Éric : «Quand on attend comme ça, ce n'est pas évident. On vit d'espoir ». L'espoir leur permet de tenir le coup malgré les tourmentes de l'incertitude. Ce thème découle des quatre sous-thèmes suivants : l'omniprésence de la mort, affronter les épreuves, les stratégies de protection et la puissance du soutien, détaillés dans les lignes qui suivent.

Le premier sous-thème, l'omniprésence de la mort, met en évidence que le risque de mourir demeure en filigrane tout au long du processus de l'attente. La mort constitue une éventualité qui peut survenir pendant l'attente et se retrouve au premier plan dans les préoccupations des participants. Cette possibilité ne peut être mise de côté puisqu'elle fait partie intégrante du risque d'attendre une transplantation. Les propos de Pierre expriment bien sa prise de conscience du danger de mourir à court terme et de se préparer à cette éventualité : «Dans ma période d'attente, nous sommes allés rencontrer le notaire. J'ai tout préparé ma mort bien froidement. Mes assurances, tout le kit, ma femme savait tout quoi faire. J'ai regardé ça bien froidement ».

De plus, pour un des participants, l'éventualité de la mort a été plus que présente au moment où il a fait un arrêt cardiaque, comme le raconte Éric :

Une journée entre autres, il était 15;30 15;45, j'étais sur le cour mécanique et je me suis levé debout pour dire bonjour à l'infirmière et là j'ai dit : "Oups, je me sens étourdi». Elle a dit : "C'est impossible avec un caur mécanique, c'est impossible »! Et là, j'ai été malade en masse. Ils m'ont réanimé et tout le kit.

Bien que les risques de mort soient omniprésents lors de l'attente, les participants ont 
profondément conscience que ces risques le sont également lors de l'intervention chirurgicale pour la transplantation. En effet, les professionnels de la santé leur fournissent toutes les informations pertinentes sur les risques chirurgicaux lors des rencontres prégreffe. Lors de l'appel pour la transplantation, cette possibilité a frappé Paul de plein fouet :

Il y a tellement de choses qui te passent par la tête. C'est comme si ta vie défilait. Tu ne sais pas si tu vas revenir. Tu penses à ça, là tu y penses. Ils vont m'endormir et je ne me réveillerai peut-être plus, tout ça, ça passe.

L'omniprésence de la mort tout au long de l'attente et particulièrement durant la chirurgie, fait peur à Hélène : «J'ai peur de mourir sur la table d'opération ». Par contre, les autres participants ne semblent pas effrayés par l'idée de la mort, comme le rapporte Pierre : «On va loin, mais je n'ai pas peur de la mort, ça ne me gêne pas de le dire ». Bien qu'il se dise prêt à mourir, la possibilité de devoir abandonner les siens constitue pour Éric, l'aspect le plus difficile de l'attente :

Moi, ce qui était le plus dur, c'est que je laissais tout mon monde à côté de moi. Moi, partir, ça ne me dérangeait pas, mais laisser tous mes amis, ma femme, mes frères, mon père, ma mère. Je trouvais ça dur, mais le reste, ça ne me dérangeait pas de partir. Moi, j'étais prêt.

La mort tisse la toile de fond de la période d'attente de la transplantation. Les participants sont conscients qu'il s'agit d'une éventualité tangible lors de l'attente; cependant, celle-ci ne leur fait pas peur malgré tout. Au contraire, ils sont prêts à cette éventualité et c'est plutôt la possibilité de quitter ceux qu'ils aiment qui est rapportée comme la plus difficile à vivre. En plus de cette éventualité difficile à concevoir, de nombreuses épreuves viendront parsemer leur expérience de l'attente. 
Par ailleurs, le deuxième sous-thème, intitulé affronter les épreuves, aborde les multiples difficultés rencontrées lors de l'attente. L'incertitude se reflète de différentes façons et est vécue de manière distincte pour chacun des participants. L'incertitude face à ce que leur réserve l'avenir occasionne un questionnement constant chez eux. Des interrogations à savoir s'ils vont recevoir leur transplantation et si leur état de santé va leur permettre d'attendre jusqu'à ce qu'un donneur compatible soit identifié et que leur tour soit venu sont bien présentes en période d'attente de greffe. Ces interrogations sont d'autant plus vives lorsque le temps passe et que l'appel ne vient toujours pas. Mario rapporte ce questionnement comme un élément crucial de sa période d'attente :

Mais la vraie attente, une fois que tu es sur la liste, c'est le questionnement pour savoir comment ça va prendre de temps et est-ce qu'il va arriver quelque chose en attendant. Plus le temps allonge et tu as de plus en plus de questionnements : "C'est donc bien long, je ne suis pas compatible avec grand monde ».

Cet espoir de recevoir l'appel de confirmation se situe au cœur de l'expérience d'attendre une transplantation. Les participants souhaitent ardemment que le téléphone sonne pour les aviser qu'un organe compatible vient de leur être attribué. Le verbatim de Paul rapporte comment il vit l'expérience d'attendre cet appel et la charge émotive qui y est associée :

J'attends encore... Parfois je suis dans ma chambre et ma sæur appelle et je réponds tout de suite. Elle me dit : "Est-ce que tu attends un téléphone? " Non! Ça fait juste trois ans et demi que j'attends un téléphone!!! C'est certain que j'attends un téléphone... Je l'attends toujours, l'osti de téléphone... mais c'est jamais le bon!

Bien que le stress accompagne les participants tout au long de l'attente, celui-ci semble culminer lors de la réception de l'appel qui confirme que la chirurgie aura lieu dans les 
heures qui suivent. Dès lors, il s'agit d'un moment d'angoisse important. Lors de cet appel, Paul exprime ainsi le stress qu'il a éprouvé à ce moment :

Quand ils t'appellent, tu vires en rond, tu veux aller te laver et ça fait 10 minutes que tu es dans l'entrée de la porte de la chambre de bain et tu vires comme un fou... Tu vires en rond, tu es tellement sur les nerfs que tu n'es pas capable de rien faire. Même si tu sais que tu dois aller prendre une douche. Tu ne sais plus, tu n'as jamais vécu ça, tu ne sais pas où tu t'en vas.

Lors de la réception de l'appel qui annonce l'imminence de la greffe, un sentiment d'ambivalence se manifeste également, révélant la dichotomie entre l'angoisse ressentie face à l'issue de la chirurgie et le sentiment de joie de recevoir enfin son organe, comme le raconte Michel : «J'étais nerveux. (silence) J'étais nerveux, angoissé. C'est embêtant comment je peux te dire ça? D'un côté, j'étais content ». À la lumière de l'analyse du contenu des entretiens des sept participants, le fait de ressentir de l'angoisse a été rapporté uniquement à ce moment de l'attente, sauf par Hélène :

J'ai encore de la difficulté (silence) à m'imaginer que je vais passer par là. C'est une grosse affaire. J'ai beaucoup de craintes encore. J'ai eu beaucoup, j'ai eu des craintes. J'en ai de moins en moins, mais j'aimerais... Je trouve que c'est une grosse chose à passer.

Par ailleurs, la peur de refuser la transplantation lors de l'appel a été soulevée par Paul.

Ce dernier explique la peur qui le tenaillait face à sa réaction possible lors de la réception de l'appel, et ce, depuis le début de son attente :

Quand il t'appelle, moi j'ai toujours eu peur de ça. Ma plus grande peur, c'est quand il va appeler et qu'il va me dire: "On a des poumons pour toi, est-ce que tu viens? "J'ai toujours eu peur de ça depuis le début. Parce qu'on ne sait pas comment on va réagir, c'est impossible. Moi j'appréhendais ça, je ne savais pas comment j'allais réagir. 
Attendre l'appel qui les mènera à la transplantation fait partie intégrante de l'expérience d'attendre une greffe. Au cours des entretiens, les participants ont soulevé plusieurs éléments qui caractérisent cette période. Tout d'abord, ce moment est espéré depuis qu'ils savent qu'ils doivent recevoir une greffe ce qui, pour certains, représente des mois, voire même des années d'attente. Toutefois, la peur de ne jamais le recevoir occasionne un effet de surprise lorsque cet appel survient, comme le décrit Mario : « Heille! Tu fais un saut! ». Il s'agit là d'un moment fort en émotions et qui le demeure même lorsque les participants évoquent ce souvenir. En effet, les participants qui ont vécu ce moment ont tous eu la larme à l'œil lorsqu'ils se sont remémorés cette période particulière de leur attente. Cet appel représente à la fois pour eux l'espoir de vivre et la possibilité de mourir. Éric explique en ces termes son incrédulité quand les professionnels lui ont annoncé qu'il avait son cœur :

Ils m'ont dit que j'allais avoir un cour et là, c'est resté comme ça. Quand le médecin est arrivé, mon cardiologue, a dit : "Avez-vous appelé votre femme?" J'ai dit: "Non, je l'ai pas appelée. La dernière fois, on était presque rendus sur la table quand on a été refusés ». Il a dit : "Non, appelez, vous allez avoir votre cour ce matin ». Là, ça a comme déboulé, on était contents, on espérait.

Malheureusement, il arrive trop souvent que cet appel tant attendu ne se fasse pas entendre. De nombreuses raisons peuvent expliquer le silence du téléphone tels le nombre peu élevé de donneurs, les difficultés de compatibilité et la qualité des organes prélevés. Éric n'a jamais su les raisons de ce silence, mais il était au courant lorsque l'équipe refusait les cœurs. Ces refus signifiaient pour lui un temps d'attente qui continuait de s'étirer, ce qui détruisait son moral et amenuisait son espoir de recevoir un nouveau cœur : 
Ils en ont refusé plusieurs, cinq à six (cœurs) certain qu'on en a refusés, qui ne faisaient pas. Dans ce temps-là, tu retombes encore dans la cave comme on dit, parce que le moral n'est plus là. Tu penses que tu vas être greffé assez vite, mais ce n'est pas le cas. Il faut attendre, c'est ça qui est le plus difficile.

De plus, l'appel tant convoité était parfois pour un autre patient qui attendait le même organe. De voir une autre personne recevoir cet appel d'espoir a rendu cette expérience encore plus amère pour Paul et a suscité chez lui un sentiment de désespoir, comme le traduisent les lignes qui suivent :

Une autre chose qui nous affecte beaucoup... Je le sais moi, ça fait plusieurs fois que j'aurais dî être greffé depuis que je suis ici, parce que mon groupe sanguin, c'est un qui est le plus commun. Ils en ont greffé comme ça (en montrant une main ouverte vers le haut) à ma place. Moi, quand c'est mon tour, ils ne me greffent pas et ils en greffent un autre. Pourquoi? Parce qu'ils sont en urgence. Alors quand c'est mon tour, non ils ne me greffent pas. Ils en greffent un en urgence. Ça fait un voyage de ça et ça aussi c'est arrivé en même temps (l'annulation de la chirurgie de la transplantation) alors tout ça mis en semble, là, j'ai pété au froid.

L'attente de l'appel de la transplantation peut également générer de la colère et de l'incertitude, comme le décrit Hélène :

Je ne comprends pas. Nous avons téléphoné à l'équipe de greffe et ils nous ont dit qu'ils respectaient la priorité. Pourtant, je l'ai vue (une autre patiente en attente) il n'y a pas longtemps et elle allait beaucoup mieux que moi. Je suis fâchée, il va arriver quoi maintenant? Qui va passer avant moi encore? Est-ce que mon tour va finir par arriver?

Ce moment rempli d'espoir de la possibilité de prolonger sa vie peut donc être également suivi d'une grande déception. L'appel de la transplantation n'est pas garant que celle-ci sera réalisée puisqu'il est toujours possible qu'à l'arrivée au centre hospitalier, le participant se voit refuser l'organe pour de nombreuses raisons. Paul et Éric ont vécu ce refus de dernière minute. Ils qualifient ce moment comme étant la pire épreuve qu'ils aient 
vécue. Pour Paul, devoir refaire le processus de préparation et sortir de l'hôpital sans sa transplantation a été très difficile à vivre :

Une autre chose qui est difficile, c'est quand ils t'appellent pour la greffe, qu'ils te préparent toute sur la table et ils te disent que c'est cancellé, c'est difficile. Je l'ai vécu, il y a deux semaines. Tabarnack, ça là, ouffffff...Tu ne peux pas blâmer personne parce que c'est tout un coup de téléphone, ça. C'est ta vie qui est mise en cause. C'est assez fatiguant mettons... et là, il faut que je recommence... Là, ils viennent de me "clancher " une fausse alerte en plus. Elle est difficile à avaler, celle-là...

Éric a vécu ce refus comme une énorme déception et même comme un choc pour lui et sa famille :

Tout le monde à côté de moi, ils ont été... Ç'a été vraiment une brique qui est tombée sur tout le monde. On était déçus. On pleurait. Ç'a été une super de grosse déception qu'on a eue là. Se faire dire qu'on allait avoir de quoi et on n'a rien. On a attendu toute la nuit, éveillés, tout le monde parce qu'on était contents, on fêtait quasiment. On était fiers. Là, on arrivait au bout du chemin, on était rendus là. On a su ça le matin (en parlant du refus de transplantation), c'est spécial.

L'annulation de la chirurgie pour la transplantation est une épreuve qui ébranle les participants et amenuise leur espoir de se voir offrir le cadeau de la vie. De plus, pour Hélène et Pierre, l'attitude des autres envers eux constitue une épreuve en soi. En effet, ces deux participants ont éprouvé de la difficulté avec les commentaires formulés par des connaissances.

Pour certaines personnes entourant les participants, la possibilité d'une transplantation pour sauver leur vie n'était pas un choix à faire. Pierre a vécu cette expérience à quelques reprises lors de ses sorties; il rencontrait alors des personnes qui lui disaient qu'il allait mourir. Ce commentaire a été reçu difficilement par Pierre. Le peu d'espoir face à la transplantation manifesté par les connaissances de Pierre l'a affecté au point qu'il ne 
voulait plus sortir de la maison. Cette attitude négative face à la greffe avait pour conséquence de mettre en doute l'espoir qu'il avait d'échapper à la mort. Il a d'ailleurs été dans l'obligation d'expliquer le processus de transplantation à plusieurs personnes afin de ne plus être confronté au doute que suscitaient les commentaires négatifs. L'extrait suivant évoque ce qu'il a vécu :

Moi, ce que j'ai trouvé le plus dur dans l'attente, ce n'est pas moi-même, mais les autres. Les autres me disaient : "Pauvre Pierre!". J'ai des copains et des clients qui me disaient : "Pauvre Pierre, tu vas mourir!". Je disais : "Pis, je suis rendu au bout! Est-ce que tu sais quand tu vas mourir toi? "Il répondait : "Non!",, alors je disais : "Tu vas peut-être mourir demain matin ». [...] Il y a beaucoup de personnes... mon deuxième voisin m'a dit: "Je ne crois pas ça, les greffes, tu vas mourir ». C'est difficile de se faire dire ça. Je lui avais dit: "J'ai jasé avec mes cardiologues et j'ai des bonnes nouvelles. Ils m'ont dit qu'il n'y avait aucun problème qu'ils allaient me greffer un caur. J'ai fait un "deal " avec en haut. Je vais vivre deux semaines de plus que vous, vous allez mourir avant moi ». Il a fermé sa gueule et il ne m'a jamais reparlé. J'ai trouvé ça difficile. Les gens me disaient: "Ça ne marchera pas, c'est des cochonneries. Tu vas souffrir pour rien $"$.

Quant à Hélène, c'est l'incompréhension des autres face à la gravité de son état de santé qui l'a mise en colère. Lors de la visite de ses proches à son domicile, ceux-ci ont minimisé la gravité de la situation et ne semblaient pas comprendre ce qu'impliquait pour elle de vivre dans l'attente d'une transplantation. Cette incompréhension a été difficile à vivre pour Hélène, comme elle le souligne :

Les gens, ils pensent que c'est facile. Tu vas à l'hôpital. Ils changent ça et tu reviens à la maison. Salut bonjour, c'est fait! Mais ce n'est pas si facile que ça. Ils me disent que je ne suis pas si pire que ça, que ma santé va s'améliorer.

En conclusion, le second sous-thème, affronter les épreuves, traduit les moments que les participants ciblent comme étant les plus difficiles lors de l'attente de la transplantation. Vivre de l'incertitude, de l'incompréhension et de la colère ne facilite en rien cette attente. 
L'espoir de vivre représente donc une force leur permettant de poursuivre cette attente, comme en témoigne Michel. Pour celui-ci, il a été plus difficile de garder le moral, mais l'espoir de la greffe l'a aidé à tenir le coup : «Le moral n'était pas fort, mais d'un autre côté, je savais qu'il allait se passer quelque chose, alors ça m'aidait à tenir le coup ». L'espoir de vivre est primordial pour aider les participants à passer à travers cette étape, comme le soulève aussi Pierre : "L'attente, c'est garder le moral pour réussir à passer au travers et se rendre à la greffe ". Le verbatim de Mario exprime également l'importance de l'espoir : «L'espoir est important pour continuer et se rendre à la greffe ». Afin de garder espoir pendant cette attente remplie d'embûches et qui leur semble si longue, les participants élaborent des stratégies de protection qui leur permettent de traverser leur quotidien.

Le troisième sous-thème, les stratégies de protection, aborde les moyens utilisés par les participants afin de conserver cet espoir qui leur permet de passer à travers la période d'attente le mieux possible. Tous les participants ont développé diverses stratégies de protection auxquelles ils ont eu recours selon les aléas de leur cheminement, afin de se tenir à flot et de ne pas sombrer. Tout d'abord, demeurer positifs face à l'avenir leur permet de se rendre à la greffe et de ne pas se décourager. Pour Daniel, il s'agit de la stratégie à adopter : «Je me disais : «Il faut que je reste positif ». Jusqu’à maintenant, ça bien été. J'ai toujours gardé le moral. J'étais confiant mais je veux dire...Je me disais qu'il faut que je reste positif ».

Pour sa part, Paul s'est imposé de ne pas penser à sa situation afin de ne pas se décourager. Il a choisi, volontairement et consciemment, de faire abstraction de sa situation 
pour s'assurer une stabilité psychologique qui lui a permis de garder la tête hors de l'eau. Il a été plus facile pour lui d'ignorer certains éléments de son quotidien, comme le révèle cet extrait :

Le pire, le pire... Attendre, c'est rien. C'est quelque chose là, mais on n'y pense pas. On se fait comme une carapace. On ne pense pas à ça, on déjeune, on dine, on soupe et on se couche. Notre attente c'est ça, on ne s'arrête pas à penser: $j$ 'attends, j'attends, parce qu'on va se pendre dans la garde-robe. [...] On ne pense pas à notre cas et il ne faut pas, parce qu'on s'apitoierait sur nous autres. On va virer fou.

Dans le même sens, il a évité de poser trop de questions afin de conserver son optimisme : "Tu es impuissant devant ça. Si tu te mets à poser toutes sortes de questions, tu n'auras plus confiance en rien. Tu vas virer fou. Moi ma job, c'est t'attendre, alors j'attends ».

Pour se protéger de la colère ressentie lors de la greffe de quelqu'un d'autre, Hélène a décidé de s'isoler. L'extrait suivant témoigne de cette stratégie : «Non, je suis un peu sauvageonne. Je me renferme beaucoup alors, plutôt d'achaler les gens, je me rentre dans ma coquille. Je vis ça seule».

Par ailleurs, l'expérience des autres personnes en attente de transplantation produit un impact chez les participants de l'étude. D'un côté, une expérience positive de transplantation leur permet de demeurer optimistes, comme le fait remarquer Daniel : «Je voyais qu'il y avait beaucoup de monde qui avait eu des greffes et ça se passait bien ». D'un autre côté, se comparer positivement avec d'autres personnes qui sont dans une condition plus piètre que la leur les aide à garder espoir, comme le décrit Paul : « Compte tenu de la maladie que j'ai, ça va bien. Il y en a des pires que moi. Il y en a qui ne 
marchent plus ».

Dans un autre ordre d'idées, le fait de voir un ami quitter la Maison des greffés du Québec pour recevoir sa transplantation apporte du réconfort à Paul : « À la limite, on pourrait les envier. Mais ce n'est pas ça, ce n'est pas un monde comme ça. À chaque fois qu'il y en a un qui part pour une greffe, on est tout content de ça».

Adopter des stratégies de protection permet donc aux participants de garder espoir et de faire face à l'attente de la transplantation. Plusieurs stratégies ont été développées, que ce soit en demeurant positif face à l'avenir, en faisant abstraction de sa situation ou en évitant les questions pour ne pas avoir à faire face à des éléments plus négatifs. Une autre stratégie utilisée par les participants en contact avec d'autres candidats à la transplantation consiste à centrer leur attention sur ceux dont l'épisode de greffe s'est bien déroulé. Enfin, ils font également abstraction des évènements négatifs vécus, que ces derniers soient les leurs ou ceux des autres candidats.

Le dernier sous-thème, la puissance du soutien, représente un élément incontournable de l'expérience de l'attente pour les participants de l'étude. Le conjoint, les proches et les professionnels de la santé représentent ce soutien, de même que la foi pour l'un d'entre eux. Les participants vivant en couple soulèvent tous que, sans l'aide et le soutien de leur conjoint, il leur aurait été impossible de passer à travers cette attente, comme en témoigne Pierre : « Je vais être honnête avec vous. Si je n'avais pas eu de support de ma femme et de mes enfants, je n'aurais pas passé au travers. Elle a été d'un support extraordinaire ».

La présence du conjoint fournit un soutien tangible et, parfois, il aide même le participant à ne pas abandonner, comme le soulève Éric : 
Ma conjointe... (pleure) ma conjointe m'a supplié tout au long de ma greffe. Elle a été là tous les jours, tous les jours avec moi. Je ne pouvais pas laisser passer ça. Je devais être là encore un bout avec elle. C'est ce qui m'a aidé le plus à passer ma greffe, en attente et tout. C'est ce qui m'a aidé beaucoup.

Le soutien d'un conjoint apparaît prépondérant dans le partage des responsabilités qui s'impose lors de l'attente de la greffe. Paul, qui vit seul, déplore le fait de ne pouvoir compter que sur lui seul :

Donc, quand ça t'arrive, tu es tout seul pour tout régler. Il y en a qui sont accompagnés, leur conjoint est avec eux. Tu n'as pas besoin de penser à tout parce que c'est entendu. Tu as quelqu'un qui prend ta place. Mais quand tu es tout seul, tu ne peux pas donner la responsabilité à quelqu'un d'autre.

Pour sa part, l'appui fourni par son conjoint dans les activités de la vie quotidienne s'est avéré très important pour Hélène puisque sa condition de santé ne lui permettait pas de les accomplir seule. Elle expose ainsi le rôle primordial que ce dernier joue dans sa vie : «Le fait que mon conjoint soit avec moi, ça c'est certain. Il a pris sa retraite pour pouvoir s'occuper de moi ».

Bien que les conjoints fournissent une aide précieuse, les proches occupent également une place importante dans la vie des participants et leur apportent également du soutien. De même, les amis peuvent aussi avoir une influence positive sur le moral des participants, surtout lors des moments plus difficiles, comme le soulève Daniel : « On a eu une chance cette journée-là d'avoir un ami. Un de mes amis qui a monté (ville de résidence). C'est lui qui nous a parlé et qui a réussi à nous faire passer une journée un peu mieux de ce qui s'annonçait ».

La présence des amis amène également de l'énergie à Daniel : 
Juste de savoir que tous mes amis du monde allaient être contents... parce que je connais beaucoup de monde (nom de l'endroit) dans mon coin, autant que j'en ai rencontré ici. Et là je me disais : "Tout ce monde-là qui le savent ». On dirait que ça me donnait de l'énergie.

Par ailleurs, Daniel et Paul ont dû se refaire un réseau social suite à leur relocalisation à Montréal, à la Maison des greffés du Québec. Les autres candidats à la transplantation et leur aidant naturel ont donc été présents lors de moments plus difficiles. Paul relate que certains deviennent même des amis : «On se refait un autre réseau social. Surtout ici à la Maison des greffés, tu rencontres des personnes qui sont toutes dans le même bateau. On se fait des amis là-dedans ». Les propos de Daniel rendent compte de l'aspect positif de côtoyer ces personnes : «Le monde s'encourage, ceux qui ont connu l'expérience, parfois ils vont encourager ceux qui ont de la misère ".

La collaboration des professionnels de la santé, tant médecins qu'infirmières et autres intervenants, revêt aussi une grande importance dans l'expérience d'attendre une transplantation. Les participants voient leur présence comme une collaboration favorisant leur bien-être. Les mots de Pierre relatent l'importance de leur apport : « Extraordinaire (nom chirurgien)! Les infirmières, les médecins, le psychiatre, l'équipe de transplantation, ils travaillent tous ensemble. Ils ne prennent pas de décision sans se consulter. On se sent en sécurité ».

L'un des points apprécié chez les professionnels de la santé porte sur l'honnêteté avec laquelle ils répondent à leurs questions concernant leur situation. Selon Éric, cette honnêteté a fait une différence dans la considération qu'il leur porte :

J'ai été gâté, gâté. J'ai été suivi. Quand je leur demandais de quoi, ils me répondaient. Ils ne m'ont jamais fait de mensonge parce que je leur avais 
toujours dit de tout le temps me dire ce qui se passait. Ils m'ont fait pleurer souvent, mais c'était ce que je voulais, avoir toute la réalité pour savoir vraiment ce qui se passait. Quand on a refusé des cours parce qu'ils disaient que ça ne faisait pas, ben c'était ça. Ils me faisaient pleurer, mais je l'avais demandé. Mais ils ont été très, très... Ils étaient merveilleux. C'était des anges pour moi.

De plus, un autre point soulevé par les participants réside dans la confiance éprouvée envers les membres de l'équipe de soins. Selon eux, il était primordial de leur faire entièrement confiance et d'accepter de remettre leur vie entre leurs mains. Paul aborde ainsi cet aspect :

Moi ce que j'en pense, je n'en pense rien. Je ne connais rien là-dedans. Je leur ai donné les clés en 2005, alors conduis parce que moi, je ne conduis plus. Je dépends d'eux autres. Je n'irais pas leur dire comment faire leur travail. Je ne sais pas. S'ils jugent que c'est le meilleur pour moi, alors je vais prendre ça.

Dans le cas de Michel, vivre l'attente d'une transplantation a même changé sa vision du système de santé, comme le relate cet extrait d'entretien :

Ça toute changé mon opinion sur le système. Ce n'est pas vrai qu'il est à point, il manque peut-être des médecins. Dans des gros cas comme ça, ils sont là. Tu ne vas pas à l'hôpital ici pour une prise de sang parce que c'est ordinaire, mais quand c'est pour une transplantation c'est extraordinaire. Tu as énormément de personnes qui gravitent autour. Je n'ai rien de négatif à dire contre eux autres aujourd'hui.

Ainsi, les conjoints, les proches et les professionnels de la santé fournissent un soutien important dans la période de l'attente pour les participants de cette étude. L'apport de la foi a, quant à lui, été soulevé seulement par Pierre. Bien présente dans sa vie et ce, longtemps avant cette période, la foi représente une source de soutien inestimable pour lui, comme il l'exprime dans ce qui suit :

Je suis un gars excessivement croyant. Moi, ma mère était plus catholique que le Pape. J'ai toujours diné avec le frère André. Je me suis accroché énormément au frère André. Ça m'a énormément aidé dans ma démarche pour accepter tout ça, 
passer au travers de tout ça et vivre ça. Si je n'avais pas eu la foi, ça ne me gêne pas de le dire, je ne passais pas au travers.

Bref, garder l'espoir de vivre malgré la tourmente de l'incertitude lors de l'attente d'une transplantation s'avère être une période ardue pour les participants. L'incertitude de ce que l'avenir leur réserve et qui s'amplifie avec le temps, l'angoisse face à l'impact que peut représenter un simple appel dans leur vie, la présence de la colère face à la réaction des autres et le fait de constater que d'autres candidats sont greffés avant eux constituent des épreuves qui complexifient davantage l'attente et qui effritent l'espoir.

Des stratégies de protection sont donc mises en œuvre par les participants afin d'être capables de faire face à l'attente tout en gardant l'espoir que ce jour finira par arriver et leur sauvera la vie. En plus de ces stratégies, le soutien reçu semble important et même essentiel pour survivre à cette épreuve. Les participants soulèvent ce fait en nommant clairement que, sans le soutien de leur conjoint, de leur famille et de leurs amis, des professionnels de la santé et de la foi pour l'un d'entre eux, ils n'auraient sans doute pas été capables de passer à travers ce long combat qu'évoque l'attente d'une transplantation.

\section{Mettre sa vie en suspens}

Mettre sa vie en suspens représente le dernier thème de l'expérience d'attendre une transplantation. En effet, vivre dans l'attente d'une transplantation décrit un quotidien qui peut parfois être difficile et bien particulier et où le temps influence l'expérience de l'attente de chaque participant. Vivre dans le présent est difficile pour eux, puisqu'ils ne cessent de regarder vers le passé qui leur rappelle de douloureux souvenirs et n'osent anticiper le futur qui peut faire peur. Le moment présent est perçu comme un arrêt du 
temps. Paradoxalement, le temps semble également être à la fois infiniment long lors de l'attente et très court suite à l'appel. Vivre au jour le jour péniblement, être en rupture avec la vie et le paradoxe du temps sont des sous-thèmes qui reflètent ce que peuvent vivre les participants en attente de transplantation.

Le premier sous-thème, vivre au jour le jour péniblement évoque le quotidien des participants lors de leur attente. La progression de la maladie, les limitations et la prise de médication qu'elle impose rendent le quotidien de l'attente des plus difficiles pour les participants. Ainsi, face à sa fatigue et à son incapacité de se déplacer, Pierre entrevoit sa condition physique précaire avec accablement :

Je ne pensais pas qu'un être humain... Ça là!!! J'ai trouvé ça dur. Descendre en bas et prendre une heure pour monter mon escalier. Je n'en revenais pas!!! C'est effrayant comment un être humain peut descendre bas, mais ça remonte vite quand ça va bien.

L'incapacité physique peut même être importante au point d'empêcher une personne de s'occuper d'elle-même. Ayant une respiration difficile et un manque d'énergie marqué, Hélène était dans l'impossibilité d'accomplir seule ses activités de la vie quotidienne. Son témoignage reflète bien la condition physique dans laquelle elle se trouvait durant l'attente: «Je ne peux presque plus... J'ai de la difficulté à m'occuper de moi tellement que je n'ai pas d'énergie. Je manque d'air. Juste de parler, j'ai de la difficulté ». La maladie terminale les empêche de réaliser les activités qu'ils désirent, ce qui les amène à une impression négative de leur quotidien. Michel relate sa perception du déroulement de ses journées d'attente :

Eh bien, mon quotidien, il n'était pas... Il était très ordinaire. Je fonctionnais à $20 \%$ alors, je n'allais pas loin...Je ne faisais rien. J'étais assis, point final. C'est 
durant la nuit qu'ils m'ont appelé. Je ne faisais absolument rien. Je n'avais pas l'état pour le faire.

De plus, l'incapacité de Michel de pouvoir pratiquer une activité physique aussi simple que la marche a influencé négativement la perception qu'il avait de lui-même. Il exprime un sentiment de découragement face à cette prise de conscience :

Je me rappelle... J'en parlais encore à ma copine. Voilà six ans, on est allés marcher et je $m$ 'assoyais sur le gazon des voisins toutes les cinq minutes. Il y a une fois, je lui ai dit:"Va chercher la voiture, moi je ne marche plus ». Handicapé assez avancé!

Cet abandon d'activité provoqué par le manque d'énergie a généré chez Hélène le sentiment d'être inutile. La prise de conscience que son avenir n'était pas celui qu'elle avait imaginé influence négativement son moral :

Je me sens, je me sens démunie. Je me sens presque inutile. Ce n'est pas toujours facile à prendre, moi qui étais une personne très, très active. J'ai une grande maison, dans les autres pièces, $j$ 'ai des antiquités alors c'était... C'était notre projet d'avenir mon mari et moi et là, je ne peux plus rien faire. C'est difficile à gérer, à prendre. Mon moral, je me démoralise souvent. (silence) Hum... c'est ça, ce n'est pas facile.

Les problèmes respiratoires rendaient Paul très inconfortable. Il raconte comment, en période hivernale, les désagréments causés par le froid lui donnaient le sentiment d'être prisonnier :

L'hiver, c'est quand on respire. L'air froid qui entre dans les poumons, ça fait mal. Je viens trop essoufflé. Tu ne marches plus. Je ne suis plus capable de rien faire. Je ne peux pas sortir dehors. Moi, en bas de $20^{\circ} \mathrm{C}$, je ne sors plus. Je suis prisonnier dans ma chambre tout l'hiver. Je ne sors plus avant le printemps prochain.

Dans le cas de Daniel, la présence de sécrétions pulmonaires causées par sa maladie rendait sa respiration encore plus laborieuse, tel qu'il l'exprime ici : 
Le matin en me levant, $j$ 'avais tout le temps des sécrétions, alors je râlais tout le temps. Le souffle, la nuit, j'avais tout le temps un sifflement. C'est ce que les personnes me faisaient remarquer, mais je m'en rendais compte. Beaucoup de sifflements et de sécrétions, des lavements nasaux presque tous les jours. Je devais faire ça. C'est ça qui n'était pas facile.

Par ailleurs, afin de compenser le mauvais fonctionnement de l'organe malade, la médication constitue généralement un choix relativement efficace à court terme pour survivre durant l'attente et c'est le cas pour les participants de cette étude. Par contre, cette médication influence souvent négativement la condition physique du patient, compte tenu des nombreux effets secondaires qui y sont associés, comme en témoigne Paul :

Pour passer au travers de cette maladie-là, de vivre avec ça, il y a juste un truc. C'est qu'il faut baisser le pouls et la pression. En baissant le pouls et la pression, c'est le système de chauffage corporel qui ne fonctionne plus. Moi, depuis 2005, je suis à 80/48 et là, ça a baissé à 62/40 de tension artérielle. Je ne fais plus rien. Même s'il fait $25^{\circ} \mathrm{C}$ dehors, tu es gelé quand même.

De plus, le matériel d'assistance respiratoire et de dialyse nécessaire à la survie des participants en attente de transplantation pulmonaire et rénale s'est avéré une contrainte importante pour eux. En attente de transplantation rénale, Mario décrit les inconvénients de la dialyse péritonéale :

Durant la nuit, il faut être branché sur un appareil qui fait tes échanges péritonéaux. Nous avons été obligés de faire chambre à part pendant ce temps-là, un grand bout de temps, parce qu'elle n'était pas capable de dormir avec l'appareil. L'appareil fait un peu de bruit. Si tu veux uriner pendant la nuit, tu ne te débranches pas. Il faut que tu aies un pot à côté de ton lit et des choses comme ça qui sont des inconvénients. Tu apprends à vivre avec ça.

Daniel, en attente de transplantation pulmonaire, a trouvé difficile la contrainte de l'oxygénothérapie en continu. Il ne pouvait pas se déplacer sans son oxygène et le fait de devoir transporter une bombonne d'oxygène partout où il allait l'a limité dans ses activités : 
J'étais actif, je sortais, mais c'est certain qu'à un moment donné, traîner une bouteille de prête, limité dans le temps, de tout le temps vérifier. Tu ne peux pas profiter vraiment plus que d'une heure ou deux quand tu te déplaces. Tu ne profites pas vraiment non plus.

Le matériel d'assistance médicale représente donc une limitation physique pour certains participants. Alors qu'elle permet au corps de survivre et ce, malgré les malaises physiques qu'elle peut engendrer, cette assistance médicale peut également occasionner des craintes, la peur par exemple que le cœur mécanique cesse de fonctionner, comme le rapporte Éric :

Mon cæur mécanique, quand j'ai eu vraiment conscience que je l'avais, c'est quand je suis retourné aux soins progressifs. Là, il a fallu que j'apprenne à vivre avec. C'était les soirs, les nuits, c'est spécial d'entendre toujours claquer ça, parce que c'est toujours comme un battement de cour et ça claque assez fort, de dormir avec ça... C'était spécial, c'était pesant ici à l'abdomen. Ce n'était pas des positions correctes pour dormir. Ç'a été..., c'est spécial toujours écouter si la valise fonctionne parce que tu as toujours peur que ça arrête.

Cet inconfort physique est devenu encore plus présent chez Hélène qui doit prendre des analgésiques pour contrôler les douleurs qu'elle ressent à la cage thoracique : «Je suis obligée de prendre de la morphine pour passer au travers de mes journées. Sinon j'ai trop mal et je n'ai pas la force d'avoir de la douleur ». De son côté, une douleur bien présente a obligé Pierre à diminuer ses activités, ce qui a été difficile pour lui, comme le décrit son témoignage :

J'ai toujours été un petit vite dans n'importe quoi, comme s'il n'y aurait pas de lendemain. Ça, j'ai trouvé ça dur dans ma maladie. Être toujours obligé de " slaquer ». Le système m'obligeait par la force des choses. Il fallait vraiment que ça fasse mal pour que je "slaque».

Pour un des participants, l'expérience de la maladie terminale durant l'attente a été vécue différemment des autres. Nonobstant la dialyse péritonéale qu'il devait faire durant la 
nuit, son quotidien ne s'est pas trouvé chamboulé. De pouvoir vaquer à ses occupations dans le jour s'est avéré positif pour lui, comme il le relate :

C'est officiel, demeurer ici et avoir pu continuer à travailler. D'avoir mon revenu normal ça a changé, ça a favorisé toute la "game ". C'était idéal comme situation, compte tenu des circonstances. Tu continues d'avoir ton revenu, tu continues de travailler, tu continues d'avoir ton contact social au travail. Ça change toute la " game.», ça.

Durant l'attente, vivre au jour le jour représente de multiples souffrances pour le corps et l'esprit. Le quotidien des participants est limité par l'incapacité physique et l'obligation de vivre avec de nombreux inconforts. Certains d'entre eux doivent aller vivre à l'extérieur de leur région pour attendre leur transplantation. Cette relocalisation engendre un sentiment d'isolement de profonde solitude. Ainsi, l'un des participants ayant résidé à la Maison des greffés du Québec a soulevé le sentiment de solitude vécu pendant son attente. Vivre loin de sa résidence représente de devoir quitter les gens qu'il aime, générant ainsi un grand vide. Paul explique :

Premièrement, tu es loin de ton monde. Tu n'as plus d'amis, tu n'as plus de famille, tu n'as plus rien. Ils $m$ 'ont fait venir ici ça fait trois ans. Ça fait trois ans que je suis ici. J'ai de la visite quand je peux en avoir, mais à part de ça, j'en ai pas beaucoup.

Bien présente, la solitude pouvait parfois même devenir difficile pour le moral. Pierre, qui a vécu plusieurs semaines hospitalisé loin de son domicile, se trouvait loin de sa famille: «En plus, c'est loin, on est loin. Ici (région), on est loin de Québec. La visite, tu n'en as pas là-bas. Ça te prend un moral de chien là-bas à Québec. J'ai passé des semaines tout seul». 
Devoir vivre loin de chez soi occasionne des contraintes inhérentes à cette situation. Paul a vécu le deuil de son père sans pouvoir se rendre au salon funéraire puisque la trop grande distance à parcourir entre le centre hospitalier et sa ville natale ne lui aurait pas permis de recevoir sa transplantation s'il avait reçu l'appel. Il a vécu difficilement cette situation et a alors éprouvé des sentiments d'impuissance et de solitude :

Moi je suis monté ici et mon père est mort. Qu'est-ce que tu fais? Tu ne peux pas rien faire. Tu ne peux pas partir d'ici. Tu es pris ici. Tu as une personne de ta famille qui meurt et tu ne peux pas partir d'ici. Tu ne peux pas aller au service, aller voir ta famille. Tu es tout seul. C'est des choses que tu as à vivre encore tout seul. Tu ne peux pas dire: "Je vais y aller", parce que si dans le temps que tu es parti, le téléphone sonne, tu fais quoi? Il faut que tu gères ça.

Outre la solitude et l'isolement, devoir quitter sa maison peut également générer un lourd sentiment de perte, voire de deuil. Dans le cas d'Hélène, cette contrainte d'attendre loin de chez elle représentait l'éventualité de devoir faire euthanasier ses animaux. En attente à son domicile, elle devra sous peu quitter la maison familiale pour aller attendre à la Maison des greffés du Québec. Elle ne pourra emmener avec elle ses animaux qu'elle affectionne particulièrement. Elle décrit cette éventualité avec une lourde charge émotive :

C'est ça le plus difficile, que je trouve. C'est d'abandonner ma maison et mes animaux pour rester sept à huit mois, peut-être plus. Les animaux, il faut que je les fasse euthanasier. Personne ne va les garder, huit chats (rit). Ça, je trouve ça difficile de quitter la maison. L'abandonner complètement, parce que c'est loin. On s'éloigne beaucoup.

Mario, qui a attendu à son domicile, voit la relocalisation comme un facteur contribuant négativement à l'expérience de l'attente. Il exprime cette vision lorsqu'il décrit les aspects positifs d'attendre chez lui : «Ton entourage est toute là comme avant. Il n'y a rien de changé de ce côté-là. Obligé de déménager pour ça, c'est plus d'inconvénients pas 
mal ». De plus, la relocalisation à la Maison des greffés du Québec est également perçue négativement par Hélène, puisqu'elle considère ne pas être chez-elle, comme elle l'explique : «Bien, c'est une chambre. On est bien. On est bien traité, mais ça demeure une chambre. C'est comme l'hôtel. Tu n'es pas chez toi, c'est ça». Par contre, cette expérience n'a pas été vécue de la même manière pour tous. Paul, qui vit là-bas depuis presque trois ans, explique qu'il a le sentiment d'être chez lui :

Nous avons notre chambre, nos choses à nous autres. Si on n'est pas bien quelque part, on s'en vient ici (en parlant de sa chambre). Je suis chez moi ici. C'est la seule place que j'ai vue qui était comme ça. C'est beaucoup plus facile à vivre ici que chez quelqu'un d'autre.

Vivre dans une région éloignée des centres transplanteurs occasionne également des frais supplémentaires, tant pour ceux qui doivent être relocalisés que pour ceux qui ont la chance de rester à leur domicile. En effet, ces derniers doivent parcourir de nombreux kilomètres pour avoir accès aux soins de santé, engendrant ainsi des coûts élevés pour les participants. De plus, Michel soulève que, malheureusement, en plus des factures élevées, l'aide financière n'est pas disponible :

Concernant l'aide aux personnes éloignées des grands centres, elle n'était pas là. Je suis allé un peu partout avec mon beau-frère. Un peu partout dans les hôpitaux de la région pour avoir de l'aide parce que dans l'état que tu es, tu ne peux pas fonctionner. Il faut que tu coures. Je ne me voyais pas partir un mois d'ici, le loyer à payer. Tout payer et arriver à Québec parce que tu restes deux à trois semaines à tes frais, parce que rien n'était couvert. Les cardiologues ont beau dire les soins sont pareils pour tout le monde, oui, mais l'aide financière n'est pas là.

En résumé, pour les participants, vivre au jour le jour péniblement, c'est être obligés de faire le deuil de leur santé, être incapables de s'occuper d'eux et devoir recevoir des soins par leurs proches. La maladie terminale signifie également le risque bien présent 
d'une mort imminente suite à un état de santé qui ne cesse de se détériorer. Les participants vivent avec de nombreuses incapacités physiques qui les empêchent de vivre leur vie comme ils en ont envie. Pour certains, ce quotidien signifie également devoir quitter leur entourage pour se rendre à proximité d'un centre de transplantation. La relocalisation constitue un aspect significatif de l'expérience de l'attente pour certains participants. S'ensuivent donc la solitude, l'obligation de faire le deuil de leur chez soi, de leur famille et de leurs amis. De plus, certains des coûts financiers peuvent être plus importants.

Le second sous-thème, être en rupture avec la vie, est proposé puisque l'attente d'une transplantation vécue par les participants représente une rupture de leur vie. En effet, leur état de santé précaire et l'incertitude génèrent chez eux la sensation de ne pas vivre une vie à la hauteur de leurs attentes. Comme le souligne Hélène, sa vie lors de l'attente est comparable à une rupture du temps. Elle a le sentiment que le temps s'est arrêté et qu'il ne se passe plus rien. Incapable de s'occuper de ses besoins fondamentaux et de faire des loisirs, elle éprouve le sentiment de ne plus avoir de vie, de ne plus exister : «La qualité de vie, c'est zéro. Je n'existe point, je ne vis plus. Je suis en attente, ma vie maintenant, c'est avant la greffe et après la greffe. Notre vie, parce que (nom du conjoint), il vit la même chose que moi».

Lors de l'attente, l'incapacité physique liée à la maladie terminale prend une place importante dans la vie des participants, à tel point qu'ils doivent abandonner les activités qui leur permettaient de s'accomplir. Daniel, un sculpteur, n'avait plus la force de pratiquer son art puisqu'il devait concentrer toute l'énergie qu'il avait sur sa maladie : 
Quand même, au niveau créatif, moi je fais des arts, de la sculpture. J'ai tout arrêté ça. Pour moi, produire, faire de quoi dans la maladie... Il y a du monde qui, faut qu'il s'évade là-dedans. Mais moi, je me disais non...

Pour sa part, le récit de $\mathrm{Michel}$ met également en relief cette rupture avec la vie pendant l'attente et le désir de commencer une nouvelle vie grâce à la transplantation :

Ha, il y a des fois que c'est long. Parfois on a hâte... Parfois, on a hâte que ça soit fini, que tout soit réglé. Recommencer une vie normale. Ce n'est pas une vie être en attente. C'est stressant, on ne peut pas se promener. On ne peut pas aller nulle part, c'est certain qu'on n'a pas l'état. On ne peut pas partir à $500 \mathrm{~km}$ de chez nous, il faut attendre.

Pour certains participants, cette rupture de la vie se traduit par le sentiment de vivre dans un état second comme le rapporte Pierre :

Tu es comme dans un état second, c'est comme si tu fumerais. [...] Tu es comme dans un état second. J'ai l'impression que je n'étais plus moi-mêrme la journée où j'ai su que c'était la mort qui s'en venait et que j'avais besoin d'une transplantation. Il y a toujours comme un dédoublement dans notre tête. On l'accepte, on a peur, on n'a pas peur, on "deal " avec ça. C'est toute notre expérience de vie et ce qu'on a vécu et ce qu'on est, je pense, qui fait qu'on ballotte au travers de ça. Je n'ai pas d'autre... Mais on n'est pas nous autres à $100 \%$, c'est officiel. Moi, je suis certain d'une chose, si on était vraiment bien froid, on se tire au bout du pont...

Alors que pour Pierre, vivre l'attente signifie vivre dans un état second, Paul a l'impression de vivre dans un monde parallèle, dans lequel il vit une sorte d'inconscience, comme il le témoigne :

Il peut arriver n'importe quoi, sans être préparé. Il faut être inconscient pour embarquer là-dedans. Moi je me dis ça, c'est une sorte d'inconscience. Tu ne réalises pas ce qui t'arrive et c'est aussi bien comme ça. Je n'aimerais pas ça, me casser la tête et vouloir comprendre.

Paul affirme également que cet état second le contente d'une certaine façon: «Comme pour moi, je n'y pense même pas. Je ne sais pas si c'est parce que tu n'es pas conscient. Je 
suis content, tu n'es pas conscient de ce qui t'arrive, tu es chanceux". Vivre dans un état second semble une stratégie plus ou moins consciente que la personne utilise pour poursuivre le chemin de l'attente.

Bref, être en rupture avec la vie reflète la réalité de l'attente. Les participants définissent cette sensation comme le sentiment de ne plus pouvoir se réaliser, comme l'obligation de suspendre ou de mettre fin à des projets, compte tenu de leur état de santé. De plus, ils éprouvent le sentiment d'une rupture du temps, expriment qu'il ne se passe plus rien depuis qu'ils sont en attente, que tout s'est arrêté. Vivre, c'est avant ou après l'attente, mais attendre, ce n'est pas vivre, c'est plutôt vivre dans un monde parallèle où la personne se sent dans un état second.

Attendre une transplantation peut durer quelques jours, quelques mois... mais aussi des années. Le troisième sous-thème, soit : le paradoxe du temps influence grandement la perception que les participants associent à la manière dont ils vivent ou ont vécu l'attente. L'incertitude de savoir « quand » aura lieu cet appel est perceptible dans l'expérience de l'attente de la transplantation. Plus le temps s'étire, plus les participants se sentent envahis par l'incertitude et plus il devient difficile pour eux de garder espoir, tel que le relate Mario :

Il y en a que c'est deux mois, il y en a que c'est deux ans. Moi, ça a été trois ans et il y en a qui ne le seront jamais. Moi, c'était le questionnement de quand la greffe va arriver. Ça finit que c'est long, trois ans... ça fait long. Ça fait long de questionnements, mais c'est comme ça.

L'expérience d'attendre une transplantation semble interminable. Qu'elle s'échelonne sur deux mois ou trois ans, les participants ont tous le sentiment que cette attente est longue 
et ce, sans distinction quant à la durée réelle de cette dernière. Lors de l'entretien avec Éric qui avait attendu avec un cœur mécanique pendant moins de quatre mois, il exprime ce qu'il a ressenti face au temps : «Attendre pour moi, moi l'attente ça été super long. Quand même que ça n'a pas été long, 111 jours, il y en a qui attendent plus que ça».

Dans les cas de Paul et d'Hélène, ce sentiment d'attente interminable teinte fortement leur expérience, comme l'exprime Paul : « C'est long... c'est long... (moment de silence) ». Lors des remerciements pour la participation à cette étude, Hélène a également soulevé que l'attente est interminable : « Ça m'a fait plaisir, si ça peut t'aider et nous aider ceux qui attendent longtemps, parce que c'est long... et que c'est long...».

L'attente, lorsque la condition physique des participants se détériore rapidement, semble également avoir un impact sur leur perception du temps. L'état de santé précaire d'Éric le préoccupait à un tel point qu'il craignait ne pas se rendre à la transplantation si le temps se prolongeait trop. L'espoir de recevoir celle-ci se trouvait ébranlé par le temps qui filait et par l'appel qui ne venait pas. Son témoignage en fait foi :

Moi, je me voyais toujours dépérir. Est-ce qu'on va en avoir un? En attente, tu espères toujours et là, toutes les fois qu'ils disaient "On n'a pas!!! On n'a pas!!!" " Tu trouves les journées plus longues. Tu te vois baisser. Tu dis: "Est-ce qu'on va en avoir un? Est-ce qu'on va réussir à passer au travers? "Tu ne sais pas si tu vas continuer de vivre. Moi, mon attente, ça a été ça. Ça a été si on va en avoir un, à un moment donné.

Paradoxalement, malgré le sentiment que le temps soit long lors de l'attente de l'appel de la transplantation, le temps devient soudainement trop court lors de la réception de cet appel tant attendu. Daniel décrit cette expérience en soulevant le manque de temps dont il disposait pour faire un dernier téléphone aux personnes aimées : 
Il fallait que les appels soient assez courts, aux deux trois de la famille que j'ai appelés. J'ai dit: "Vous passerez le message aux autres", parce que je n'avais pas le temps d'appeler toute la gang... J'étais serré dans le temps.

Quant à Paul, ce bref moment lui a fait prendre conscience qu'il n'avait pas régularisé avec ses proches toutes les situations qu'il aurait voulu régler avant de partir pour avoir sa greffe et que le peu de temps dont il disposait était désormais trop court pour le faire :

Là, tu penses à tout, tes enfants, tes petits-enfants, tes chums. Tu aimerais pouvoir tous les rejoindre en même temps, mais tu ne peux pas. Tu n'as pas le temps parce que tu dois partir. C'est là que tu te rends compte que la vie... Il lui manque un petit bout. Tu voudrais une petite chance plus longue (rire nerveux).

Ce moment entre l'appel et la chirurgie file à toute allure. À ce propos, Michel explique qu'il n'a même pas eu le temps de se rendre compte de ce qui se passait et de réfléchir. Il aurait voulu prendre le temps de dire au revoir aux personnes qui l'accompagnaient, mais à ce moment, il a pris conscience qu'il était déjà trop tard.

En résumé, l'expérience du temps est influencée par les évènements qui jalonnent le parcours de l'attente. Mettre sa vie en suspens fait état pour les participants de la temporalité au quotidien, du fait de voir leur état de santé se détériorer et de constater que les incapacités physiques limitent chaque jour davantage leurs possibilités. C'est la rupture du temps, un sentiment que le temps s'est arrêté. Finalement, le paradoxe du temps se rapporte à une compréhension du temps qui fluctue selon le moment, celui-ci étant tour à tour trop long ou trop court.

En conclusion, l'essence phénoménologique qui exprime ce que vit un adulte en attente de transplantation est formulé ainsi : Attendre une transplantation, c'est mettre sa vie en suspens et affirmer sa volonté de vivre en gardant espoir malgré la tourmente de 
l'incertitude. La Figure 3 illustre, à l'aide des thèmes proposés plus bas, l'allégorie du vortex dans le cadre de l'attente de la transplantation.

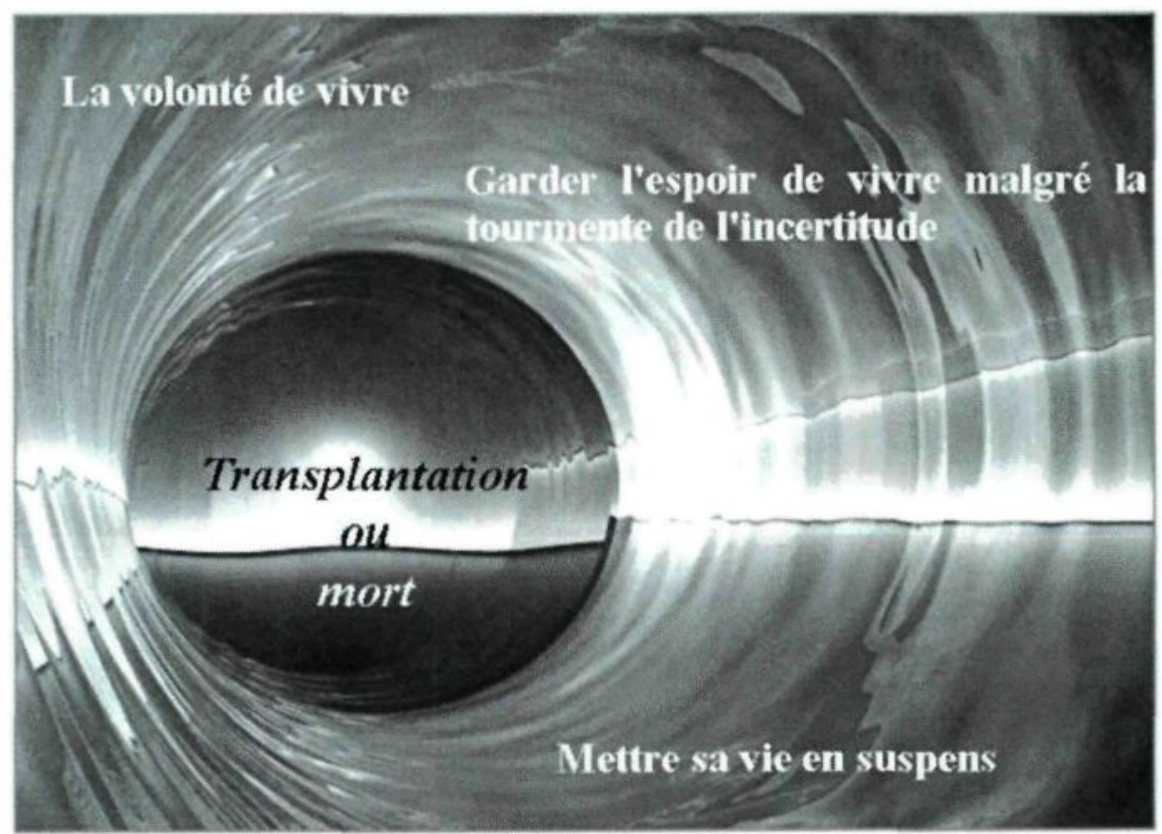

Figure 3. Essence de l'attente d'une transplantation.

Vivre l'attente, c'est se retrouver dans un vortex, à savoir dans un tourbillon qui vous entraîne irrésistiblement (Centre national de ressources textuelles et lexicales, 2012). Cette métaphore vise à rendre compte de l'absence de linéarité dans le processus de l'attente, des sentiments d'espoir et d'incertitude s'entrecroisant au fil des épreuves et des stratégies de protection. 
Ainsi, ce vortex de l'attente prend naissance dès le moment où les participants prennent la décision de vivre, c'est-à-dire de tenter la transplantation. Leur vie entière est alors suspendue dans ce vortex en mouvement où les repères d'une vie antérieure sont absents et où l'avenir est incertain. Survivre, voilà ce sur quoi ils doivent concentrer leur énergie, compte tenu des embûches qui gravitent dans ce tourbillon.

Dans ce vortex, la réalité de l'attente est longue et n'offre pas la possibilité d'un retour en arrière. Les participants doivent donc suivre le courant et se laisser emporter vers l'une des deux seules issues possibles : soit la transplantation, soit la mort. Néanmoins, ce vortex représente l'ultime porte de sortie vers un nouveau monde : un monde où l'espoir d'une vie en santé accompagne les participants dans cet ultime voyage. Le chapitre suivant aborde la discussion des résultats, les limites et forces de l'étude ainsi que des recommandations pour la pratique, la recherche et la formation infirmière. 
CHAPITRE V

DISCUSSION 
Tout d'abord, cette étude phénoménologique descriptive avait pour but de décrire et comprendre la signification de l'expérience vécue de patients en attente de transplantation vivant à l'extérieur des grands centres de la province de Québec. La question de recherche formulée afin d'atteindre le but de la recherche était : Quelle est la signification de l'expérience d'attendre une transplantation? Les résultats obtenus suite à cette question exposent une description détaillée du phénomène à l'étude soit : l'attente d'une transplantation.

Dans la section qui suit, une discussion concernant les données sociodémographiques et cliniques des participants est présentée. Les thèmes qui ont émergé des résultats sont ensuite discutés à la lumière des écrits existants.

\section{Données sociodémographiques et cliniques}

À la lecture des données sociodémographiques, il ressort que la plupart des participants, soit cinq sur sept, étaient en couple lors de l'attente de la transplantation. Il est difficile de faire une comparaison avec les autres études, puisque la plupart d'entre elles ne spécifient pas le statut matrimonial de leurs participants. De plus, aucune donnée à ce propos n'est disponible à partir des statistiques de Transplant Québec.

Le temps d'attente des participants, quant à lui, est très différent de celui rapporté par Transplant Québec (2013c). Ainsi, il est polarisé dans deux catégories spécifiques, soit un à trois mois et 13 mois et plus, contrairement à la moyenne d'attente québécoise qui se situe à six mois pour un cœur, 25 mois pour des poumons et 41 mois pour des reins (Transplant Québec, 2013b). Cette différence est peut-être liée à la faible taille de l'échantillon, soit sept participants et à la nature des catégories incluses dans le questionnaire 
sociodémographique. En effet, il aurait sans doute été plus pertinent de demander aux participants le nombre de mois pendant lequel ils ont vécu en attente, au lieu de procéder à partir d'une échelle basée sur des intervalles de temps. De cette façon, cette comparaison aurait été plus juste. De plus, deux personnes étaient toujours en attente lors de l'étude, ce qui ne permet pas de dresser un portrait fidèle du temps d'attente vécu par les participants.

Dans la présente étude, la participation a été plus importante chez les hommes (six) que chez les femmes (une). Aucune donnée n'est fournie par Transplant Québec quant à la répartition des hommes et des femmes dans la population en attente de transplantation au Québec, ce qui rend impossible l'établissement de toute comparaison à ce propos. Par ailleurs, dans les études recensées, les pourcentages d'hommes et de femmes varient considérablement, ce qui ne permet pas d'établir une relation à ce sujet entre la présente étude et celles répertoriées.

Enfin, lors des entretiens, deux des participants étaient en attente et les cinq autres avaient reçu leur transplantation. Tous les participants étaient bouleversés lors des entretiens, que ceux-ci vivent l'attente au quotidien ou évoquent les souvenirs de cette période de leur vie. Vivre l'attente d'une transplantation s'avère une expérience qui génère de nombreuses émotions et celles-ci semblent se refléter tout aussi intensément lorsqu'ils y réfèrent. Toutefois, aucune nuance n'est apportée à ce sujet dans les lignes qui suivent.

\section{L'expérience d'attendre une transplantation}

La discussion réalisée dans cette section traite des trois thèmes qui traduisent l'essence de l'expérience d'attendre une transplantation et qui ont émergé des sous-thèmes soit : la 
volonté de vivre, garder l'espoir de vivre malgré la tourmente de l'incertitude et mettre sa vie en suspens.

\section{La volonté de vivre}

Les patients prennent davantage conscience de leur volonté de vivre au moment où on leur annonce que leur mort devient imminente. Une fois l'annonce faite, ils doivent alors prendre la décision de tenter ou non la greffe.

Ce thème expose le sentiment d'arrêt de mort ressenti par les participants lors de l'annonce du pronostic fatal, à plus ou moins court terme, de leur maladie terminale et les raisons qui motivent leur prise de décision concernant la transplantation. En effet, les participants ne se croyaient pas malades au point de devoir subir une greffe pour continuer à vivre. Le pronostic de mort imminente a été pour chacun un choc important et parfois même une réalité difficile à concevoir. Contrairement à ceux vivant avec une maladie chronique depuis longtemps, le seul participant dont l'évolution de la maladie a été subite a rapporté que, pour lui, le principal choc a été de devoir faire rapidement face au deuil de sa santé. Les résultats en lien avec ce premier sous-thème corroborent ceux de plusieurs études.

Celle de Macdonald (2006) rapporte que c'est uniquement au moment de l'annonce de l'imminence de la nécessité de la greffe que les participants prennent réellement conscience de la gravité de leur état de santé, et ce, malgré que celui-ci se soit détérioré constamment. Ils entrevoient donc cette annonce comme la finitude de leur vie. Pour sa part, l'étude de Brown et al. (2006) aborde les nombreux changements de perspective (personnelle, 
relationnelle, rôle, etc.) qui surviennent lorsque la maladie chronique devient terminale, ce qui amène les participants à développer une nouvelle identité durant leur attente de la transplantation.

Le choc de l'annonce du diagnostic a aussi été rapporté dans l'étude qualitative de Blondeau, Vornax et Roy (2013) visant à explorer l'expérience de la souffrance existentielle auprès de 14 personnes atteintes d'une maladie grave. Selon ces auteurs, l'annonce du diagnostic d'une maladie grave engendre une situation de crise. La vie bascule au sens où la maladie vient soudainement prendre toute la place (Blondeau et al., 2013). De leur côté, bien qu'ils ne la qualifient pas de choc, Habraken et al. (2008) rapportent que recevoir un diagnostic de maladie terminale s'avère être une étape difficile de la vie.

Par ailleurs, dans le cadre de la présente étude, la manière dont l'un des participants a appris son diagnostic, soit de manière fortuite, en entendant discuter les médecins de son cas, a contribué à sa difficulté de prendre conscience de la sévérité de sa condition et donne à réfléchir sur l'attitude à adopter en de telles circonstances. Fait à noter, aucune des études recensées n'a rapporté cet aspect de l'expérience d'attendre une transplantation.

Bref, l'annonce du pronostic terminal de la maladie génère une situation de crise. Les personnes semblent avoir de la difficulté à intégrer cette nouvelle réalité au plan cognitif, mais cette difficulté est davantage présente chez celles n'ayant pas une longue trajectoire de maladie chronique. Elles doivent alors ajuster leur identité en conséquence tout en prenant leur décision concernant la transplantation. 
La prise de décision de continuer à vivre semble, pour la majorité des participants de la présente étude, un choix très facile à faire puisque mourir ne constitue pas une option envisageable. La transplantation est perçue positivement puisqu'elle représente une délivrance face à la mort et une possibilité d'améliorer leur qualité de vie. Ces résultats corroborent ceux obtenus dans l'étude de Bjork et Naden (2008) où plusieurs participants ont décrit leur réaction face à l'annonce de la possibilité d'une transplantation avec des termes comme bonheur, soulagement ou sentiment de joie.

Prendre conscience que quelqu'un doit mourir pour lui sauver la vie a été rapporté par un participant comme un constat difficile lors de la prise de décision pour la greffe. De leur côté, les participants de l'étude de Sadala et Stolf (2008) expriment un sentiment de culpabilité à l'évocation de cette pensée. Dans le même sens, Laforêt (2006) affirme que le fait que quelqu'un d'autre doive mourir pour qu'elles vivent affecte profondément les personnes qui attendent cet événement, soutenant que : «Certains patients s'affligent de leur « sadisme » à la veille des longs week-ends, statistiquement plus meurtriers pour les automobilistes » (p. 6).

De plus, les résultats de l'étude de Jonsén et al. (2000) mentionnent que la difficulté des participants concernant leur prise décision au regard de la greffe s'exprime dans un « marchandage avec eux-mêmes », soit de l'ambivalence et de la peur face au choix de tenter ou non la transplantation. Enfin, Brown et al. (2006) soulignent, dans leur thème « questionner le processus », que cette ambivalence concernant la décision prise perdure tout au cours de l'attente. 
Par ailleurs, c'est le désir de vivre avec leur famille qui incite les participants de cette étude à prendre la décision de tenter la greffe. La transplantation est perçue positivement puisqu'elle représente une délivrance face à la mort et une possibilité d'améliorer leur qualité de vie. Il est intéressant de constater qu'aucune des études recensées n'a soulevé les raisons qui motivent le choix des personnes à prendre la décision de tenter la transplantation.

Dans le présent projet de recherche, six participants sur sept ont mentionné qu'ils reprendraient la même décision si c'était à refaire. En effet, les inconvénients de la transplantation sont perçus moins négativement que ceux occasionnés par leur maladie chronique. Seul le participant n'ayant pas une trajectoire de maladie chronique terminale a affirmé un point de vue contraire. Cette différence de perception peut s'expliquer par le fait qu'une longue trajectoire de maladie chronique laisse le temps aux personnes de bien intégrer la gravité de leur état, comme le souligne Laforest (2006).

De plus, la prise de décision de continuer à vivre peut également être influencée par les informations fournies par les professionnels de la santé. Les informations reçues par les participants avant d'arrêter leur choix ont été suffisantes pour les aider à prendre une décision éclairée. Lorsque les informations sont jugées satisfaisantes par les patients, ceuxci envisagent la transplantation positivement et la prise de décision s'en trouve facilitée. L'étude de Moloney et al. (2007) ainsi que celle de Haugh et Salyer (2007) soulèvent également que les informations reçues des professionnels de la santé influencent positivement la prise de décision de tenter la greffe. Par contre, dans l'étude de Martin et al. (2010), les répondants mentionnent que leur incertitude est due au manque d'information. 


\section{Garder l'espoir de vivre malgré la tourmente de l'incertitude}

L'attente d'une transplantation s'avère un processus rempli d'embûches et d'incertitude. De ce fait, tous les participants affirment que sans l'espoir, il leur serait difficile de se rendre à la transplantation. Le deuxième thème évoque l'omniprésence de la mort et les nombreuses épreuves que ceux-ci doivent affronter tout au long de l'attente. À cette fin, ils doivent recourir à de nombreuses stratégies afin de conserver leur espoir. De plus, la présence et le soutien des proches leur fournissent des outils supplémentaires pour maintenir de cet espoir. Les études recensées confirment les résultats de cette étude.

Il n'existe aucune garantie qu'une transplantation ait lieu et la possibilité d'un décès demeure toujours présente. Le premier sous-thème, l'omniprésence de la mort, confirme la présence constante du spectre de la mort et la conscience qu'en ont les participants. Les résultats soulèvent que les participants prennent intrinsèquement conscience de cette possibilité et qu'ils connaissent bien les risques inhérents à la période d'attente de même qu'à la chirurgie pour la transplantation.

Lors de la prise de décision de la transplantation, la majorité d'entre eux disaient ne pas avoir peur de la mort. De leur côté, Brown et al. (2006) interprètent cette absence de peur comme un déni explicite de la mort. L'attente est alors décrite comme une bataille, voire même un «purgatoire ». Cependant, les participants de diverses autres études affirment avoir peur de la mort (Bjork, \& Naden, 2008; Brown et al., 2006; McDermott et al., 2010). Enfin, Sadala et Stolf (2008) soulignent que plus le temps d'attente s'étire, plus cette crainte s'amplifie. 
Les participants de la présente étude expriment plutôt la peur de devoir quitter ceux qu'ils aiment. Selon l'étude de Blondeau et al. (2013), la perte anticipée des relations avec leurs proches advenant leur décès constitue l'une des préoccupations liées à la souffrance existentielle des personnes vivant avec une maladie grave.

Pour sa part, le sous-thème affronter les épreuves représente l'obligation de faire face à l'incertitude et aux contraintes rencontrées tout au long de l'attente. Irvin (2001) pointe d'ailleurs l'incertitude comme étant l'une des caractéristiques de l'attente. L'incertitude face à l'avenir contribue à la détresse psychologique et au sentiment d'impuissance des personnes en attente puisqu'elles n'ont pas de contrôle sur la situation (Bjork, \& Naden, 2008; Brown et al., 2006; Fogarty, \& Cronin, 2007; Haugh, \& Salyer, 2007; Jonsén et al., 2000; Macdonald, 2006; Moran et al., 2011; Naef, \& Bournes, 2009).

L'incertitude influence donc la perception et la nature de l'expérience d'attendre des participants. Dans la présente étude, l'incertitude vécue par les participants s'exprime entre autres par le doute de ne pas savoir quand la transplantation aura lieu. Il est soulevé par la question récurrente, à laquelle il est impossible d'obtenir une réponse, à savoir : Quand vais-je recevoir un organe? Cette question, qui s'avère être un point culminant de l'incertitude, a également été rapportée dans l'étude de Haugh et Salyer (2007).

Cette incertitude s'est également manifestée chez les participants de cette étude à travers le sentiment de ne pas avoir la certitude de recevoir la transplantation en soulevant la question suivante : «Est-ce que je vais me rendre à la transplantation? ». Pour leur part, Jonsén et al. (2000) comparent cette incertitude lors de l'attente à de l'agonie. Comme Bjork et Naden (2008) le font remarquer, cette question est légitime puisque les participants 
ont un état de santé précaire pouvant les conduire à la mort ou encore à être retirés de la liste d'attente. D'ailleurs, selon les statistiques de Transplant Québec (2013c), 5,5\% des candidats à la transplantation sont décédés en 2012.

$\mathrm{Au}$ plan théorique, ces résultats fournissent des informations précieuses pour documenter le phénomène d'incertitude. D'une part, les personnes en attente d'une greffe font face à l'imprévisibilité de l'évolution de la maladie et de son pronostic, comme le mentionne Mishel (1990) dans sa théorie sur l'incertitude liée à la maladie. De plus, comme elle l'évoque, l'incertitude peut être perçue de manière négative ou positive. Dans cette étude, les participants semblent envisager la transplantation positivement. À l'instar de l'étude de Bjork et Naden (2008), avoir une vision positive de la transplantation durant l'attente leur permet de conserver leur espoir d'une vie nouvelle.

Par ailleurs, il est intéressant de faire un lien entre les résultats de cette recherche et le concept d'incertitude de Penrod (2001a). Celle-ci soulève que l'incertitude est fondée sur le doute ou sur le fait de ne pas connaître les résultats de la situation, de même qu'à la signification que la personne lui accorde. De telles perceptions engendrent un sentiment d'inconfort et mettent à l'épreuve les sentiments de confiance et de contrôle que la personne détient sur la situation, conduisant alors à différents types et modes d'incertitude. Dans cette étude, le phénomène d'attendre une transplantation engendre non seulement de l'incertitude situationnelle, laquelle est de nature épisodique et concerne un événement précis, mais également de l'incertitude existentielle. Cette dernière apparaît plus vague mais fait appel à l'introspection de la personne concernant des questions existentielles comme « Pourquoi pas moi »? lorsqu'un autre candidat reçoit sa transplantation. Par 
ailleurs, les participants vivent des moments d'incertitude « omniprésente » (pervasive uncertainty) dans laquelle ils n'ont aucun contrôle sur l'issue de l'attente. Toutefois, l'espoir leur permet de garder confiance en l'avenir bien que, au fur et à mesure que le temps d'attente s'allonge, le sentiment de confiance face à l'avenir s'effrite. Ils vivent alors de l'incertitude «écrasante» (overwelming uncertainty). Dans ce type d'incertitude, les personnes se sentent immobilisées par la prise de conscience abrupte de l'impact de l'attente sur leur existence. L'incertitude s'exprime alors par un bouleversement émotionnel (Penrod, 2001a). Ce concept de l'incertitude élaboré par Penrod (2001a) permet une meilleure compréhension de l'incertitude vécue par les participants lors de l'attente d'une transplantation.

Cette incertitude «écrasante » se reflète dans la charge émotive qu'ont exprimée tous les participants lorsqu'ils ont relaté leur expérience de l'attente. Cette tension était présente à un tel point que certains participants ont été dans l'incapacité de poursuivre leur récit. Quelques participants ont également éprouvé un sentiment de colère et d'injustice lorsqu'ils ont appris qu'une autre personne allait recevoir, avant eux, le même type d'organe dont ils avaient besoin. D'autres ont également éprouvé de la colère envers les personnes de leur entourage qui ne semblaient pas comprendre la dure réalité de l'attente vécue.

Ces résultats s'avèrent novateurs puisqu'aucune des études recensées par l'étudiante chercheure ne soulève le sentiment d'injustice éprouvé par les personnes en attente d'une transplantation de même que le sentiment de colère en réponse à l'attitude d'incompréhension manifestée par les proches lors de cette expérience. Ils mettent en évidence le bouleversement émotif lié à l'expérience de l'attente et à la méconnaissance de 
la population concernant cette expérience et la transplantation d'organes. Ces résultats soutiennent les propos d'Ellefsen (2013) à l'effet que les personnes vivant avec une maladie chronique font l'expérience d'une souffrance qui affecte profondément non seulement leur corps et leur esprit mais aussi toute leur vie, incluant leurs relations avec les autres.

Un élément nouveau, spécifique à cette étude et qui n'est pas soulevé dans les écrits recensés est l'effet de surprise ressenti par les participants lors de l'appel pour la transplantation. En effet, la plupart de ceux qui ont reçu cet appel ont décrit ce moment comme générant un effet de surprise, et ce, malgré qu'il soit attendu depuis plusieurs mois, voire même des années. Les participants vivent si intensément chaque jour l'espoir d'être appelés et, chaque soir, la déception de ne pas l'avoir été qu'au moment de l'appel, ils sont surpris que leur tour soit enfin venu. Ils prennent alors conscience que la seconde chance qu'ils espéraient depuis le tout début de l'attente est maintenant sur le point de se réaliser, et ce, même s'ils n'y croyaient presque plus.

De plus, les participants de cette étude rapportent combien ils trouvent difficile de vivre les faux appels. Lorsque la transplantation est annulée pour différentes raisons, la déception vécue est telle qu'un des participants voulait tout abandonner puisqu'il se croyait incapable de faire face à nouveau à l'appel. Ce résultat corrobore ceux de d'autres recherches puisque plusieurs études ont fait ressortir que les personnes finissent par croire qu'elles ont été oubliées sur la liste d'attente (Brown et al., 2006; Jonsén et al., 2000; Macdonald, 2006). Comme le fait remarquer Macdonald (2006), les faux appels ne sont pas rares dans le processus de transplantation. La grande déception et le stress vécus par cette 
annulation engendrent davantage d'incertitude quant au moment de la greffe et à la possibilité de trouver un autre donneur (Macdonald, 2006). Dans l'analyse de McDermott et al. (2010), cette déception face aux faux appels peut même entraîner de la dépression.

Bref, comme plusieurs auteurs l'affirment, l'incertitude doit être reconnue comme un problème persistant et complexe, qui a le potentiel d'agir comme un facteur de stress psychologique important (Bjork, \& Naden, 2008; Brown et al., 2006; Fogarty, \& Cronin, 2007; Haugh, \& Salyer, 2007; Jonsén et al., 2000; Macdonald, 2006; Moran et al., 2011; Naef, \& Bournes, 2009). C'est donc l'espoir qui constitue la poutre qui soutient l'effort d'attendre patiemment et avec sérénité (Bjork, \& Naden, 2008). L'expérience de vivre l'attente s'avère être éprouvante pour les participants de ce projet de recherche. Ils vivent des évènements qui viennent chambouler leur quotidien et qui le rendent plus difficile. Afin de se protéger et de minimiser les effets négatifs de l'attente sur leur vie, ils développent des stratégies de protection.

Les stratégies de protection représentent les moyens que les participants ont trouvés afin de conserver l'espoir qui leur permettra de se rendre à la transplantation. Dans la présente étude, l'espoir est décrit comme primordial pour la survie des participants. L'importance de l'espoir est aussi soulevée dans la majorité des études recensées (Brown et al., 2006; Jonsén et al., 2000; Moran et al., 2011; Naef, \& Bournes, 2009). Les participants sont conscients de l'influence positive que l'espoir exerce sur eux. C'est pourquoi ils font appel à diverses stratégies pour se protéger des nombreuses épreuves à vivre durant l'attente. 
Tel que mentionné dans l'étude de Jonsén et al. (2000), les êtres humains ont une bonne capacité d'adaptation face à des situations difficiles. Ainsi, ils s'efforcent de maintenir une pensée positive en centrant leur attention sur les candidats dont l'épisode de greffe s'est bien déroulé ou en se comparant positivement à ceux dont la condition de santé est plus mauvaise que la leur. Plusieurs auteurs ont également remarqué l'importance d'adopter une attitude positive durant l'attente (Brown et al., 2006; Jonsén et al., 2000; Moloney et al., 2007). Par ailleurs, les participants, de la présente étude, tentent de ne pas penser à leur situation ou de faire abstraction des événements négatifs vécus, que ces derniers soient les leurs ou ceux des autres candidats. L'un d'entre eux affirme même que, s'il s'attardait trop à penser, il risquerait de se pendre dans sa garde-robe. En accord avec Brown et al. (2006), ce résultat exprime bien l'importance d'utiliser le déni conscient pendant l'attente afin de survivre jusqu'à la greffe.

La puissance du soutien, tant en termes d'assurer le quotidien et les soins physiques qu'en ce qui a trait au soutien moral, constitue incontestablement l'un des éléments qui permet aux candidats d'une part, de se rendre à la greffe sans perdre espoir et, d'autre part, de se rétablir dans les meilleures conditions possibles, une fois la greffe réalisée. Les participants soulèvent qu'effectivement le soutien du conjoint, des proches ainsi que des professionnels de la santé est un élément positif qui facilite la période d'attente. Il appert en effet que les relations avec les autres peuvent s'avérer édifiantes, encourageantes et génératrices d'espoir (Naef, \& Bournes, 2009). Comme le soulignent Rodrigue, Widows et Baz (2006), le réseau du soutien s'avère primordial pour assurer un appui adéquat au futur transplanté. 
En ce qui concerne le soutien des conjoints, les participants en couple rapportent que, sans l'aide de leur conjoint, cette attente aurait sans doute été impossible et que celui-ci leur a permis de tenir le coup. Contrairement à l'étude de McDermott et al. (2010), les participants de cette étude n'ont pas soulevé avoir ressenti de la part de leur conjoint cette difficulté de concilier leur vie avec leur changement de rôle de conjoint à soignant. Cette différence peut s'expliquer par le fait que les conjoints ne faisaient pas partie des personnes ciblées par l'étude et que, en conséquence, cette question n'a pas été approfondie. De plus, l'aidant naturel ne veut peut-être pas verbaliser ses états d'âme pour ne pas ajouter un fardeau supplémentaire au patient. Par contre, une des participantes a nommé le sentiment de culpabilité qu'elle avait de faire vivre cette obligation de soins à son conjoint.

Par ailleurs, lors de l'un des entretiens, une conjointe était présente et ce, malgré la lettre d'information qui mentionnait qu'il s'agissait d'un entretien individuel. Avec l'accord du participant, l'entretien a quand même eu lieu et sa conjointe s'est exprimée sur le vécu de ce dernier même si les questions aient été posées au participant. En accord avec Burker et al. (2006), vivre l'attente de la transplantation d'un conjoint peut s'avérer être une expérience des plus complexes et comporte une charge émotive également bien présente. Les aidants naturels sont trop souvent dans l'ombre de la personne malade. Ils peuvent avoir l'impression d'être ignorés et mis à l'écart (Haugh, \& Salyer, 2007). En effet, les soins de santé et l'attention sont dirigés vers le patient et laissent trop souvent de côté les besoins de l'aidant naturel (Burker et al., 2006).

De plus, les participants ayant vécu l'attente à la Maison des greffés sont davantage confrontés au vécu des autres candidats à la transplantation. Cet aspect peut s'avérer positif 
en termes de soutien entre personnes vivant des expériences similaires, mais il peut également avoir des effets négatifs pour le participant. Ainsi, lorsque ce dernier voit d'autres candidats recevoir l'appel de la transplantation, cela lui donne espoir qu'un jour, son tour viendra. Les participants de l'étude de Haugh et Salyer (2007) ont fait ressortir que la possibilité de côtoyer d'autres personnes en attente ou qui ont reçu une transplantation les a encouragés et soutenus, en plus de leur permettre de partager ce qu'ils vivaient. Par contre, dans la présente étude, s'il s'agit d'un candidat en attente du même organe, cet espoir est ébranlé, car le participant éprouve l'impression qu'on a pris sa place. Un sentiment de colère et d'injustice émerge alors et affecte grandement l'espoir qu'un jour, c'est lui qui sera appelé. Cet aspect unique et fondamental de l'attente n'a été évoqué dans aucune des études recensées, d'où l'aspect novateur de la présente étude.

Les participants ont également soulevé l'importance qu'ils attribuent aux soins fournis par les professionnels de la santé. Comme ils l'ont mentionné, ils doivent faire preuve d'une confiance presque aveugle à leur égard puisqu'ils remettent leur vie entre leurs mains. Ils ont aussi mis l'accent sur l'honnêteté avec laquelle ils les ont informés sur leur état de santé. Bref, cette attitude des professionnels favorise l'impression d'être pris en considération et le sentiment de ne pas être qu'un numéro. À l'instar de Haugh et Salyer (2007), maintenir la dignité et le respect des personnes en attente constitue une intervention efficace et utile.

En résumé, pour les participants de cette étude, le temps qui se prolonge amenuise l'espoir de recevoir l'appel de la transplantation. Cet espoir est ébranlé par l'omniprésence de la mort et les nombreuses épreuves qui parsèment leur attente. Afin de le conserver, ils 
doivent développer des stratégies pour se protéger et la présence du soutien atténue les conséquences de ces épreuves. Ces résultats corroborent ceux de plusieurs études (Burker et al., 2006; Haugh, \& Salyer, 2007; McDermott et al., 2010; Naef, \& Bournes, 2009; Rodrigue et al., 2006).

\section{Mettre sa vie en suspens}

Pour les participants, l'attente d'une transplantation représente une opportunité de prolonger leur vie. Cependant, cette période s'avère difficile puisque de nombreuses épreuves apparaissent tout au long du processus. Ils doivent donc vivre au jour le jour avec le lot de contraintes imposées par une maladie terminale. Cette réalité engendre également un sentiment de rupture avec la vie. Enfin, le présent apparaît paradoxal puisqu'il semble à la fois trop long ou trop court lors de l'attente. Ce troisième et dernier thème discute de vivre au jour le jour péniblement, d'être en rupture avec la vie et le paradoxe du temps.

Le premier sous-thème, vivre au jour le jour péniblement, signifie que les participants sont confrontés aux conséquences de la maladie terminale dans leur quotidien. Ils expriment à quel point ils ont ressenti de la fatigue et un manque d'énergie important. De plus, leur état de santé leur occasionne de nombreuses incapacités physiques avec pour conséquence une diminution de leur qualité de vie, comme le rapportent également d'autres études (Bjork, \& Naden, 2008; Limbos et al., 2000). Enfin, selon Limbos et al. (2000), les patients en attente de transplantation ont généralement une qualité de vie inférieure à celle rapportée en postgreffe. 
Les participants de la présente étude mentionnent qu'en plus des limitations physiques engendrées par la maladie, la médication et les appareils d'assistance sont également des facteurs qui limitent leur capacité physique, générant par le fait même une souffrance psychologique. L'étude de Ek et Ternestedt (2008) rapporte que la médication en lien avec la maladie peut parfois entraîner des effets secondaires plus importants que les symptômes de la maladie elle-même, induisant des inconforts et parfois même de la douleur physique et une souffrance psychologique.

Il semble que les participants de la présente étude qui ont un état de santé précaire éprouvent davantage de difficulté à conserver le moral et une vision positive de leur situation. De plus, si leur état de santé se dégrade, leur moral se détériore également. Suite à l'entretien de validation, l'étudiante chercheure a pu constater que le moral d'un des participants était passablement moins bon que lors de la première rencontre. En effet, suite à de nombreuses hospitalisations qui ont conduit à des intubations, la participante affirmait qu'elle était beaucoup plus démoralisée, que l'espoir de recevoir sa transplantation s'en trouvait amoindri et qu'elle entrevoyait difficilement la lumière au bout du tunnel. Ces résultats font aussi écho aux propos de Bjork et Naden (2008) ainsi qu'à ceux de Sadala et Stolf (2008), qui rapportent que les limitations physiques importantes vécues par les personnes en attente de transplantation génèrent une grande souffrance.

La maladie terminale induit également un sentiment de solitude chez les participants et celui-ci se répercute sur leur état de santé mentale. Cette solitude est liée soit à une hospitalisation ou à la perte des contacts sociaux habituels. Dans le même sens, Diaz- 
Dominguez et al. (2006) expliquent ce sentiment de solitude par l'isolement social et par la perte des loisirs que les répondants avaient l'habitude de pratiquer.

En ce qui concerne la relocalisation, les participants ont mentionné qu'elle signifie d'être coupés de leur famille, de leurs amis et de leur milieu. Bien que résider à la Maison des greffés du Québec leur ait permis de se constituer un nouveau réseau social, ils ressentent tout de même un sentiment de perte et de solitude. Même si la relocalisation comporte des avantages, comme le soutien social apporté par les autres candidats en attente, être témoin de la détérioration de l'état de santé des autres candidats ou de l'appel d'un autre candidat pour la transplantation du même organe que celui qu'eux-mêmes attendent constitue néanmoins un désavantage. Des difficultés financières peuvent également résulter de cette relocalisation. Or, aucune des études recensées ne fait état des avantages et des désavantages de la relocalisation lors de l'attente.

Enfin, toutes les études recensées ont été réalisées chez des patients nécessitant la greffe d'un organe spécifique tels les reins, le foie, les poumons, mais aucune ne compare la réalité d'attendre une transplantation en fonction du type d'organe à recevoir. Cette réalité est-elle différente selon le type d'organe attendu ou si cette particularité importe peu? Dans cette étude, le choix des participants ne s'est pas appuyé sur la nature de l'organe à recevoir et aucune spécificité n'a été reliée au type d'organe à recevoir. Le vécu des participants, qu'ils soient en attente de cœur, de poumons ou de reins, s'est avéré généralement similaire.

Le deuxième sous-thème, Être en rupture avec la vie, a été évoqué puisque les participants ont rapporté avoir eu l'impression que leur vie est mise en suspens lors de leur 
attente. L'arrêt de leurs projets, de ce qui donne un sens à leur vie, et l'incapacité physique ressentie contribuent à générer cette sensation d'être en rupture avec la vie. Le temps semble s'arrêter et leur vie est mise «sur pause ». Ils deviennent nostalgiques du passé mais gardent espoir face au futur. Ce sentiment d'une vie en suspens a également été mis en évidence dans d'autres études (Moran et al., 2011; Sadala, \& Stolf, 2008). La période d'attente est thématisée par Macdonald (2006) comme une « vie dans les limbes ». Cette métaphore représente bien le sentiment vécu par les participants lors de l'attente. Ils ont l'impression que leur vie est entre deux mondes, soit celui de l'avant transplantation et celui tant espéré de l'après. Selon le philosophe Porée (2000), la souffrance altère le sens du présent auquel la personne réfère pour distinguer un avant et un après. Elle génère la déchronologisation du temps, soit les références données au temps. Par conséquent, la différence entre le « hier », « aujourd'hui » et « demain » n'existe plus. Bref, le « présent » de l'attente est un « temps à part » comme Brown et al. (2006) l'affirment.

Cette impression de vie en suspens correspond à une perte de contrôle sur les évènements et à un sentiment de manquer de liberté (Bjork, \& Naden, 2008). Selon Penrod (2001a), durant les moments d'incertitude « écrasante », le temps se perd dans l'expérience. Il n'y a aucun futur ni passé, juste un présent dans lequel le temps semble interrompu. La personne se concentre alors sur le présent pour tenir à distance le futur incertain et diminuer son sentiment d'impuissance.

Par ailleurs, cette mise en suspens de la vie est si présente que, pour certains participants, ils ont le sentiment ne plus exister ou encore d'évoluer dans un monde parallèle. Dans son analyse du concept de l'attente, Irvin (2001) soulève que, lors d'une 
attente prolongée, les patients peuvent avoir une perception du temps incongrue avec le temps réel.

Le paradoxe du temps. La perception du temps s'avère un élément important de l'expérience d'attendre une transplantation. Les participants rapportent un paradoxe du temps vécu durant leur attente, comme le font également ceux de l'étude de Brown et al. (2006). En effet, le temps apparaît trop long lors de l'attente, sans distinction quant à la durée réelle de ce dernier.

Toutefois, une fois l'appel reçu, le temps devient soudainement trop court. Les participants avouent manquer de temps pour les choses qu'ils auraient pu entreprendre pendant la période de l'attente de l'appel et qu'ils n'ont pas faites. Les propos de Porée (2000) concernant la souffrance pourraient proposer une explication à cette situation puisqu'ils postulent que le temps perçu en souffrance est un temps sans échéances. La souffrance est le point où le temps et l'éternité se touchent (Porée, 2002). Plus spécifiquement, Ellefsen (2013) fait remarquer que la souffrance vécue par les personnes vivant avec une maladie chronique apparait interminable. Cette perception subjective du temps infini pourrait ainsi expliquer l'inactivité vécue. L'appel vient donc confronter les participants à la réalité du temps chronologique.

Cette attitude de ne pas régler toutes leurs choses à l'avance soulève également quelques interrogations. Ainsi, il est plausible de se demander si cette manière de réagir est en congruence avec le fait de conjurer le mauvais sort. En effet, peut-être qu'être trop prêt pourrait signifier que l'appel a moins de chances d'arriver? De même, peut-être qu'on ne se prépare pas pour une chose dont on n'a pas la certitude qu'elle va arriver. 
En conclusion, le dernier thème mettre sa vie en suspens reflète ce que représente de vivre au jour le jour péniblement avec une maladie terminale, d'être en rupture avec la vie et de percevoir le temps différemment lors de l'attente.

La représentation de l'attente d'une transplantation en utilisant l'analogie du vortex (voir p. 95) vise à faire ressortir, de manière symbolique, le tourbillon dans lequel s'engage la personne qui fait le choix d'entreprendre le processus d'attendre une greffe. De nombreuses forces en présence s'affrontent au quotidien et le patient s'engage sur une voie de non retour où deux issues seulement sont possibles, la mort ou la transplantation. Elle s'avère novatrice puisqu'aucune des études recensées n'a proposé de métaphore pour illustrer la tourmente que constitue l'attente d'une transplantation. Cette mince contribution constituera peut-être les prémices d'un modèle plus complet dont l'utilisation pourrait permettre de faire avancer les connaissances sur le vécu des personnes en attente de transplantation.

La section qui suit présente les limites et les forces de cette étude. De plus, elle fait état des recommandations pour la pratique, la recherche et la formation infirmière.

\section{Limites et forces}

Cette section expose de manière globale les limites et les forces relatives à cette étude.

\section{Limites}

Cette étude présente cependant certaines limites, la principale étant liée à la méthode de recrutement. L'étudiante chercheure demeurant dans une région éloignée, elle n'avait 
pas aussi facilement accès aux patients en attente de transplantation puisque ces chirurgies se pratiquent dans les centres hospitaliers de grands centres urbains.

Une seconde limite de l'étude est liée à la charge émotive vécue par les participants. L'étudiante chercheure avait prévu cette possibilité en assurant un soutien psychologique à chaque participant, suite à l'entretien. Par contre, celle-ci n'avait pas envisagé que cette charge émotive rendrait l'entretien plus difficile. Certaines questions sont donc restées en suspens afin de ne pas les bouleverser davantage.

Il importe aussi de soulever que l'atteinte de la redondance des données n'a pas été totalement réalisée. À cette fin, il aurait été intéressant de pouvoir recruter un nombre plus élevé de participants, même si aucun nouveau sous-thème n'a été ajouté lors du dernier entretien. Par ailleurs, certains éléments auraient pu être questionnés un peu plus en profondeur pour obtenir une compréhension plus riche du phénomène.

Une autre limite de l'étude se rapporte au fait que, lors de la validation des analyses, deux des sept participants n'ont pas pu participer puisque leur état de santé ne leur permettait pas une seconde rencontre, l'un étant hospitalisé et l'autre devant être hospitalisé sous peu. De plus, lors de l'entretien de validation d'une participante, son état de santé était très précaire. La validation s'est faite difficilement puisque celle-ci était presque incapable de parler suite à sa dyspnée et à la prise régulière de morphine pour soulager sa douleur.

Les résultats rapportés mettent en évidence l'expérience des participants qui ont vécu ou qui vivent l'attente. Cet élément peut contribuer aux limites de cette étude puisqu'il s'avère ici impossible de faire une distinction entre vivre l'attente au présent et référer aux souvenirs d'un évènement passé, alors que la greffe a eu lieu. 
Malgré les limites de l'étude, celle-ci offre une description détaillée de l'expérience d'être un adulte en attente de transplantation au Québec. Les résultats tentent d'apporter une contribution significative à la compréhension de l'expérience d'attendre une transplantation.

\section{Forces}

L'approche qualitative a été favorisée afin de mieux comprendre le vécu des adultes en attente de transplantation. Ce choix s'est avéré judicieux puisque le but de la recherche qualitative est de mettre l'accent sur la compréhension approfondie et élargie d'un phénomène complexe et peu connu (Fortin, 2010), ce à quoi correspond en tous points le vécu des patients en attente de transplantation. Cette étude basée sur l'approche phénoménologique a donc permis de réaliser une description du vécu de sept candidats ayant vécu l'attente d'une transplantation vivant à l'extérieur des grands centres de la province de Québec. La diversité des organes à recevoir, le lieu d'attente et le temps d'attente ont permis une description riche du phénomène.

\section{Recommandations}

Suite à l'analyse des résultats, certaines recommandations peuvent être formulées concernant la pratique, la recherche et la formation infirmière.

\section{Pour la pratique}

À l'instar d'Ellefsen (2013), l'attente d'une transplantation apparait une expérience affective pénible associée à la perte de son identité. Elle touche profondément les personnes 
dans tous les aspects de leur vie (leur corps physique et mental, leurs relations avec les autres et leur relation avec le temps). Comme l'incertitude fait partie intégrante de ce tourment émotif, il s'avère incontournable de les aider à tolérer cette incertitude (Haugh, \& Salyer, 2007). En ce sens, Penrod (2007) suggère de développer des interventions spécifiques qui rehaussent les sentiments de confiance et de contrôle chez les patients. $\grave{A}$ cet effet, il apparaît essentiel d'encourager les patients à choisir et à utiliser différents moyens pour conserver leur condition physique et mentale tout au long de leur attente. Comme Macdonald (2006) le rapporte, ces mesures permettent d'《 acheter du temps » en vue de la transplantation, laquelle peut survenir très tardivement dans le processus de l'attente.

Les résultats de cette étude indiquent également que la compréhension de la maladie et de la situation influence les prises de décision chez les patients en attente d'une transplantation. Les infirmières doivent donc s'assurer d'ajuster les informations transmises en fonction du rythme et de l'intérêt des patients (Haugh, \& Salyer, 2007). Par ailleurs, il est essentiel de leur fournir des informations véridiques quant à leur situation de santé tout en les respectant et en les intégrant dans la prise de décision lors des rencontres multidisciplinaires (Haugh \& Salyer, 2007).

De plus, de nombreuses stratégies de protection ont été mentionnées par les patients en attente d'une transplantation pour conserver leur espoir et réussir à passer à travers ce processus. Entre autres, la pensée positive et l'utilisation consciente du déni devraient être encouragés pour soutenir cet espoir et recadrer l'incertitude (Brown et al,, 2006). Il est aussi recommandé que les infirmières et les professionnels qui dispensent des soins à ces 
personnes leur offrent un soutien émotif par une écoute active et empathique leur permettant de verbaliser sur ce qu'elles vivent (Ellefsen, 2013; Macdonald, 2006). Dans cette optique, des questions réflexives comme «Qu'est-ce qui est le plus difficile en ce moment? » semblent pertinentes pour favoriser l'expression des pertes accompagnant la souffrance des personnes vivant avec une maladie chronique terminale (Ellefsen, 2013). D'autres interventions peuvent aussi être mises de l'avant pour maintenir l'espoir tout au cours du processus d'attente. Notons entre autres, les aider à se centrer sur le présent, établir des buts à courts terme ou recourir à l'humour (Sacchse, 2007).

Ce soutien émotif devrait également être apporté aux proches tout au long de ce processus puisqu'ils doivent faire face eux aussi à la dure réalité de l'attente. Les impliquer davantage dans les soins leur permettrait de se sentir utiles et d'avoir le sentiment d'être pris en considération (Haugh, \& Salyer, 2007). L'adoption d'une approche systémique familiale (Wright, \& Leahey, 2007) s'avère donc un incontournable dans les soins à prodiguer aux personnes qui attendent une transplantation. Enfin, la formation de groupes de soutien pour les personnes en attente d'une transplantation et leurs proches pourrait contribuer à diminuer le sentiment d'isolement vécu durant ce processus qui semble interminable.

\section{Pour la recherche}

Lors de recherches ultérieures, il serait intéressant d'explorer le vécu des conjoints des patients en attente de transplantation. Il serait en effet à propos de documenter la charge émotive vécue par les conjoints de même que leur perception face à l'incertitude et à la 
détérioration de l'état de santé du patient. De plus, cette recherche permettrait d'évaluer le soutien qui leur est offert et favoriserait l'amélioration de la pratique infirmière en matière d'intervention auprès de cette clientèle.

Il serait également intéressant d'effectuer une étude comparative entre le vécu de patients atteints d'une maladie chronique terminale et celui de ceux souffrant d'une maladie terminale d'apparition subite, les deux groupes étant en attente de transplantation. Cette comparaison permettrait de soulever les différences dans la compréhension du vécu et d'adapter ensuite les soins et les services selon cette particularité.

La signification de l'expérience de l'attente d'une transplantation vécue dans le moment présent ou relatée à l'aide de souvenirs n'a pas fait l'objet de distinction dans ce projet de recherche. Cependant, il serait pertinent de faire une étude qui permettrait de vérifier si la signification de l'expérience est perçue différemment lorsque celle-ci est relatée soit au présent, soit au passé. À cette fin, une entrevue pourrait être réalisée pendant l'attente, puis à nouveau en postgreffe.

Être relocalisé durant l'attente d'une transplantation comporte certes des côtés positifs, mais l'éloignement apporte également son lot de contraintes. Il serait pertinent d'approfondir la compréhension de cet aspect spécifique de l'expérience de l'attente, en particulier en ce qui concerne les avantages et les inconvénients de vivre en communauté avec d'autres candidats à la transplantation. Pour ce faire, une étude comparative mettant l'accent sur les réalités de l'attente à domicile ou en relocalisation serait pertinente et pourrait amener des pistes de solution afin de répondre aux besoins de ces patients. 


\section{Pour la formation infirmière}

Une meilleure compréhension de l'expérience de vivre l'attente d'une transplantation permettra de développer de nouvelles perspectives de soins non seulement pour la discipline infirmière, mais également pour les autres professionnels de la santé.

Afin d'assurer une uniformité dans la pratique infirmière, il serait pertinent de fournir aux infirmières des divers milieux de soins en contact avec des patients en attente de transplantation ou greffés les connaissances appropriées sur la réalité vécue et sur les interventions pertinentes à poser pour les soutenir adéquatement. Enfin, les curriculums universitaires des professionnels de la santé qui interagissent avec des personnes en attente ou greffées devraient inclure une formation spécifique quant à leurs caractéristiques et à leurs besoins. 
CONCLUSION 
L'attente d'une transplantation s'avère une expérience qui est méconnue, tant de la population que de la majorité des professionnels de la santé. La transplantation représente, pour plusieurs personnes, la seule option de traitement pour mettre fin à une longue trajectoire de maladie chronique en phase terminale ou, de façon plus exceptionnelle, à une maladie aiguë terminale. Elle constitue la possibilité de prolonger leur vie et d'augmenter leur qualité de vie. Cependant, vivre dans l'attente de recevoir un organe affecte grandement l'expérience de l'individu, et ce, dans plusieurs sphères de sa vie.

Le but de cette étude visait à décrire et comprendre la signification de l'expérience vécue de patients en attente de transplantation vivant à l'extérieur des grands centres de la province de Québec. Cette recherche phénoménologique a en effet tenté de répondre à la question suivante : Quelle est la signification de l'expérience d'attendre une transplantation? Les résultats recueillis grâce aux sept entretiens ont permis d'enrichir les connaissances à ce propos.

Lorsqu'un patient reçoit l'annonce de la maladie terminale, il la perçoit comme un choc. Cependant, c'est souvent à partir de ce moment qu'il prend conscience de sa volonté de vivre. La transplantation est perçue comme une possibilité de prolonger sa vie et, à ce moment, il prend la décision de tenter de continuer à vivre. Une fois cette décision prise, l'attente de la transplantation commence. L'espoir influence positivement cette attente et prend son importance face à l'incertitude vécue, à l'omniprésence de la mort et aux épreuves qu'il faut surmonter tout au long du parcours. Afin de préserver l'espoir, les patients vont adopter des stratégies de protection, en plus de compter sur le soutien des proches qui affecte positivement leur expérience. De plus, vivre au jour le jour péniblement 
avec une maladie terminale constitue une expérience éprouvante. Le sentiment de voir sa vie mise en suspens est au cœur de l'expérience de l'attente d'une transplantation.

La métaphore du vortex de l'essence de l'attente d'une transplantation illustre bien cette réalité. Prendre la décision de vivre l'attente d'une transplantation, c'est faire le choix de plonger dans un tourbillon ou s'entrecroisent l'espoir et l'incertitude tout au long du processus. Malgré la présence constante du spectre de la mort, ce choix s'avère la seule possibilité afin d'accéder à une vie en santé.

Enfin, les résultats de cette étude ont favorisé la compréhension de cette réalité au Québec puisque peu de recherches sur la question ont été effectuées. Cette étude a également permis de fournir des connaissances aux professionnels de la santé afin qu'ils puissent ajuster leur pratique, en plus de dégager des recommandations pour la pratique, la recherche et la formation infirmière. 
RÉFÉRENCES 
Achille, M. (2002). Les enjeux psychologiques de la greffe d'organe. Psychologie Québec, $18(1), 18-21$.

Ashcroft, P. (2009). Adapting patient education for potential liver transplant recipients in a climate of chronic donor organ shortfall. Progress in Transplantation, 19, 59-63.

Bailey Jr, D. E., Wallace, M., \& Mishel, M. H. (2007). Watching, waiting and uncertainty in prostate cancer. Journal of Clinical Nursing, 16, 734-741.

Baran, D. (2008). Reprendre son souffle : Les bienfaits de la transplantation. Le Médecin du Québec, 43 (4), 67-72.

Beyeler, F., \& Riggenbach, C. (2012). Alain est décédé alors qu'il attendait un organe : Informations au sujet du don d'organes. Swisstransplant. Page consultée le 26 févirer 2013, à www.swisstransplant.org

Biomédecine. (2010). L'histoire de la greffe. Page consultée le 12 novembre 2010, à www.dondorganes.fr/L-histoire-de-la-greffe.html

Bjork, I. T., \& Naden, D. (2008). Patients' experiences of waiting for a liver transplantation. Nursing Inquiry, 15, 289-298.

Blondeau, D., Vonarx, N., \& Roy, L. (2013). Souffrance existentielle : De la crise à l'hyperquestionnement. APORIA, 5(1), 32-41.

Brown, J., Sorrell, J. H., McClaren, J., \& Creswell, J. W. (2006). Waiting for a liver transplant. Qualitative Health Research, 16(1), 119-136.

Burker, E. J., Evon, D. M., Ascari, J. C., Loiselle, M. M., Finkel, J. B., \& Mill, M. R. (2006). Relationship between coping and depression in heart transplant candidates and their spouses. Progress in Transplantation, 16, 215-221. 
Cazale, L., Laprise, P., \& Nanhou, V. (2009). Maladies chroniques au Québec et au Canada : Évolution récente et comparaisons régionales. Page consultée le 3 décembre 2011, http://www.stat.gouv.qc.ca/publications/santé/pdf2009/zoom_santé_janv09.pdf

Centre national de ressources textuelles et lexicales. (2012). Lexicographie. Page consultée le 20 décembre 2013, à www.cnrtl.fr/definition/vortex

Colaizzi, P. F. (1978). Psycholgical research as the phenomenologist views it. Dans Valle, R.S., King, M. Existential-Phenomenological alternatives for psychology (p. 48-71): New York : Oxford University press.

Corruble, E., Durrbach, A., Charpentier, B., Lang, P., Amidi, S., Dezamis, A., Barry, C., \& Falissard, B. (2010). Progressive increase of anxiety and depression in patients waiting for a kidney transplantation. Behavioral Medicine, 36(1), 32-36.

Cyr, L. (2010). La Maison des greffés du Québec. Page consultée le 29 novembre 2010, à http://www.maisondesgreffes.com

Diaz-Dominguez, R., Perez-Bernal, J., Perez-San-Gregorio, M. A., \& Martin-Rodriguez, A. (2006). Quality of life in patients with kidney, liver or heart failure during the waiting list period. Transplant Progress, 38, 2459-2461.

Ek, K., \& Ternestedt, B.-M. (2008). Living with chronic obstructive pulmonary disease at the end of life: A phenomenological study. Journal of Advanced Nursing, 62, 470478.

Ellefsen, E. (2013). L'impossible guérison. Vivre avec la maladie chronique. Spiritualitésanté, 6(2), 38-41.

Flamme, N. E., Terry, C. L., \& Helft, P. R. (2008). The influence of psychosocial evaluation on candidacy for liver transplantation. Progress in Transplantation, 18, 89-96. 
Fogarty, C., \& Cronin, P. (2007). Waiting for healthcare : A concept analysis. Journal of Advanced Nursing, 61, 463-471.

Fortin, F. (2010). Fondements et étapes du processus de recherche : Méthodes quantitatives et qualitatives. Montréal : Qc : Chenelière Éducation Inc.

Giorgi, A. (1997). De la méthode phénoménologique utilisée comme mode de recherche qualitative en sciences humaines : Théorie, pratique et évaluation. Dans J. Poupart, L. H. Groulx, J. P. Deslauriers, A. Laperrière, R. Mayer \& A. P. Pires. La recherche qualitative. Enjeux épistémologiques et méthodologiques. (p. 341-364). Montréal : Gaëtan Morin.

Habraken, J. M., Pols, J., Bindels, P. J., \& Willems, D. L. (2008). The silence of patients with end-stage : A qualitative study. British Journal of General Practice, 58, 844849 .

Haugh, K. H., \& Salyer, J. (2007). Needs of patients and families during the wait for a donor heart. Heart \& Lung, 36, 319-329.

Irvin, S. K. (2001). Waiting : Concept analysis. Nursing Diagnosis, 12(4), 128-136.

Jonsén, E., Athlin, E., \& Suhr, O. B. (2000). Waiting for a liver transplant : The experience of patients with familial amyloidotic polyneuropathy. Journal of Clinical Nursing, $9(1), 63-70$.

Junius, B. (2010). Rapport annuel 2009-2010. Page consultée 23 novembre 2010, à http://www.quebec-

transplant.qc.ca/QuebecTransplant_fr/PDF/Rapport_annuel_2009_2010.pdf

Kurz, J. M., \& Cavanaugh, J. C. (2001). A qualitative study of stress and coping strategies used by well spouses of lung transplant candidates. Families, Systems \& Health, $19(2), 181-195$.

Laforêt, E. (2006). En quoi les représentations sociales à l'oeuvre dans la perception du don d'organes facilitent-elles ou limitent-elles les prélèvements? Éthique et santé-Réseau Rodin. Page consultée le 26 juin 2013, à http://ccsd11.ccsd.cnrs.fr/?q=node/839 
Lasker, J. N., Sogolow, E. D., Olenik, J. M., Sass, D. A., \& Weinrieb, R. M. (2010). Uncertainty and liver transplantation : Women with primary biliary cirrhosis before and after transplant. Women and Health, 50, 359-375.

Lavigne, S. (2012). Présentation transplant Québec. Communication présentée dans le cadre du cours SSOI474, pratique infirmières en soins critiques, Chicoutimi, Université du Québec à Chicoutimi.

Limbos, M. M., Joyce, D. P., Chan, C. K. N., \& Kesten, S. (2000). Psychological functioning and quality of life in lung transplant candidates and recipients. Journal of the American College of Chest Physicians, 118, 408-416.

Loiselle, C. G., Profetto-McGrath, J., Polit, D. F., \& Beck, C. T. (2007). Méthodes de recherche en sciences infirmières: Approches quantitatives et qualitatives. SaintLaurent: QC : ERPI.

Macdonald, K. (2006). Living in limbo : Patients with cystic fibrosis waiting for transplant. British Journal of Nursing, 5(10), 566-572.

Martin, S. C., Stone, A. M., Scott, A. M., \& Brashers, D. E. (2010). Medical, personal, and social forms of uncertainty across the transplantation trajectory. Qualitative Health Research, 20(2), 182-196.

McDermott, A., Hardy, J., \& McCurry, M. (2010). Emotional impact on patients and families on the heart transplant waiting list. British Journal of Cardiac Nursing, 5 , $280-284$.

Mishel, M. H. (1984). Perceived uncertainty and stress in illness. Research in Nursing and Health, 7, 163-171.

Mishel, M. H. (1988). Uncertainty in Illness. Journal of Nursing Scholarship, 20, 225-232.

Mishel, M. H. (1990). Reconceptualization of the uncertainty in illness theory. Journal of Nursing Scholarship, 22, 256-262. 
Moloney, S., Cicutto, L., Hutcheon, M., \& Singer, L. (2007). Deciding about lung transplantation : Informational needs of patients and support persons. Progress in Transplantation, 17, 183-192.

Moran, A., Scott, A., \& Darbyshire, P. (2011). Waiting for a kidney transplant : Patients' experiences of haemodialysis therapy. Journal of Advanced Nursing, 67, 501-509.

Naef, R., \& Bournes, D. (2009). The lived experience of waiting : A Parse method study. Nursing Science Quarterly, 22(2), 141-153.

Penrod, J. (2001a). The advancement of the concept of uncertainty using phenomenological methods. (Thèse de Doctorat), The Pensylvania State University, Pensylvanie.

Penrod, J. (2001b). Refinement of the concept of uncertainty. Journal of Advanced Nursing, 34, 238-245.

Penrod, J. (2007). Living with uncertainty : Concept advancement. Journal of Advanced Nursing, 57, 658-667.

Porée, J. (2000. Souffrance et temps. Communication présentée Cercle de réflexion universitaire du Lycée Chateaubriand à Rennes, Université de Rennes.

Porée, J. (2002). L'épreuve du temps : Soufrance et maintien de la personne. 2(76), 17-32. Repéré à http://www.cairn.info/revue-societes-2002-2-page17.htm

Rodrigue, J. R., Widows, M. R., \& Baz, M. A. (2006). Caregivers of lung transplant candidates : Do they benefit when the patient is receiving psychological services. Progress in Transplantation, 16, 336-342.

Sacchse, D. (2007). Hope : More than a refuge in a storm. A concept analysis using the Wilson Method and the Norris Method. The International Journal of Psychiatric Nursing Research, 13, 1546-1553. 
Sadala, M. L. A., \& Stolf, N. A. G. (2008). Heart transplantation experiences : A phenomenological approach. Journal of Nursing and Healthcare Chronic Illnesses, 17, 217-225.

Santé et Services sociaux du Québec. (2005). Rapport national sur l'état de santé de la population du Québec. Page consultée le 5 janvier 2014, à http://publications.msss.gouv.qc.ca/acrobat/f/documentation/2004/04-228-01.pdf

Savoie-Zajc, L. (2003). L'entrevue semi-dirigée. Recherche sociale : De la problématique à la collecte des données. Sainte-Foy: Presses de l'Université du Québec.

Stubblefields, C., \& Murray, R. L. (2002). Waiting for lung transplantation : Family experiences of relocation. Pediatric Nursing, 28, 800-804.

Telles-Correia, D., Barbosa, A., Mega, I., \& Monteiro, E. (2009). Importance of depression and active coping in liver transplant candidates' quality of life. Progress in Transplantation, 19, 85-89.

Transplant Québec. (2013a). Don d'organes. Page consultée le 8 juillet 2013, à http://transplantquebec.ca/don-dorganes

Transplant Québec. (2013b). Personne en attente : Les centres de transplantation. Page consultée le 5 juillet 2013, à http://www.quebectransplant.qc.ca/QuebecTransplant_fr/centreTx.htm

Transplant Québec. (2013c). Statistiques officielles 2012. Page consultée le 10 septembre 2013, à www.transplantquebec.ca/sites/default/files/statistiques2012.pdf

Wright, L., \& Leahey, M. (2007). L'infirmière et la famille. Montréal : Qc : Édition du Renouveau Pédagogique.

Young, L. E., Molzahn, A., Starzomski, R., \& Budz, B. (2010). Families and heart transplantation : Reversing the trajectory of end stage heart disease. Canadian Journal of Cardiovascular Nursing., 20(2), 6-17. 


\begin{abstract}
APPENDICE A
LETTRE DE DEMANDE D'AUTORISATION DE RECRUTEMENT ET D'INFORMATION SUR LE DÉROULEMENT DE LA COLLECTE DE DONNÉES AUPRÈS DE LA MAISON DES GREFFÉS DU QUÉBEC
\end{abstract}


Saguenay, Qué.

Le 15 mai 2012

Madame Micheline Cyr Asselin

Directrice générale de la Maison des greffés du Québec

1989 , rue Sherbrooke Est

Montréal, Québec,

H2K 1B8

Objet Demande d'autorisation de recrutement et d'information sur le déroulement de la collecte de données auprès des clients de la maison des greffés du Québec.

Madame,

Je suis étudiante à la maîtrise en sciences infirmières à l'Université du Québec à Chicoutimi et j'en suis à la rédaction de mon mémoire dont le titre est Exploration du vécu des patients en attente de transplantation vivant à l'extérieur des grands centres. L'objectif visé est de mieux comprendre leur réalité d'être un patient en attente de transplantation et, pour ce faire, la personne qui la vit est sans contredit la mieux placée pour parler de ce que représente cette pénible expérience.

Quelques études ont démontré que ces patients vivent des moments difficiles et ce, tant au plan psychologique, que physique et financier. Ces problématiques sont par ailleurs bien souvent majorées par la relocalisation à laquelle ils doivent faire face pour pouvoir recevoir leur organe, puisque le délai alloué pour se rendre au centre de greffe est court. Or, au Québec, les centres transplanteurs sont polarisés dans deux grandes villes, soit Montréal et Québec. Les patients vivant à l'extérieur de ces centres urbains doivent donc trouver un gîte près de leur centre hospitalier transplanteur.

Dans cette situation, la Maison des greffés s'avère l'endroit qui accueille une bonne partie de cette population, du moins à Montréal. C'est pourquoi je sollicite votre autorisation afin de pouvoir procéder au recrutement des patients faisant partie de mon échantillon à la Maison des greffés, ce qui me faciliterait grandement l'accès à ces personnes qu'il serait fort difficile de joindre autrement. 
Le recrutement à la maison des greffés se ferait avec votre collaboration. Étant donné qu'il est important que les patients se sentent libres de choisir de faire partie de cette étude, il serait fort apprécié que vous acceptiez de faire la première approche. Je vous fournirai une affiche d'information sur la recherche à venir qu'il serait souhaitable que vous placiez dans une pièce commune de la Maison des greffés. Par la suite, vous pourriez demander aux patients présents s'ils ont vu l'affiche et s'ils désirent être contactés pour recevoir davantage d'information. Dans le cas d'une réponse affirmative, demandez-lui s'il accepte que vous me transmettiez ses coordonnées et je me chargerai ensuite de lui téléphoner, au moment convenu au préalable, afin de lui fournir toutes les informations nécessaires.

Voici toutefois quelques informations dont vous pourriez avoir besoin pour répondre à leurs questions :

- Il s'agit d'une entrevue individuelle (participant et étudiante chercheure), pour laquelle je serai sur place;

- D'une durée de plus ou moins 60 minutes;

- Des questions ouvertes leur seront posées pour leur permettre de s'exprimer sur la réalité qu'ils vivent. Décrire ce qu'ils pensent, ce qu'ils ont vécu ou ce qu'ils vivent;

- Il n'y a pas de bonne ou de mauvaise réponse;

- Ils pourront se retirer en tout temps du projet et peuvent refuser de répondre à certaines questions s'ils le désirent.

Les participants recherchés pour ce projet sont les patients en attente de transplantation d'organe et ceux ayant reçu une greffe d'organe depuis moins de 12 mois. Le sexe, l'âge ainsi que le type d'organe n'a pas d'importance dans la sélection des participants. Par contre, ils doivent parler et comprendre le français.

Une explication plus en profondeur sera fournie au patient avant de débuter l'entrevue et un formulaire de consentement sera signé afin de m'assurer d'une participation éclairée et volontaire. De plus, j'aurai pris contact avec les personnes responsables d'assurer le soutien psychologique au programme de greffe de chaque centre transplanteur afin de m'assurer de leur disponibilité au besoin. 
J'espère que cette lettre vous a fourni les renseignements dont vous aurez besoin. Dans le cas contraire, n'hésitez pas à communiquer avec moi et il me fera plaisir de répondre à vos questions.

Je vous remercie à l'avance de votre inestimable soutien à l'avancement de mon projet et vous prie de recevoir, madame Cyr Asselin, l'expression de mes salutations les plus distinguées.

Geneviève Taché, B. Sc. inf. Étudiante à la maîtrise en Sciences infirmières Université du Québec à Chicoutimi Tel : 418-815-2863

Courriel : genevieve.tache@uqac.ca 


\section{APPENDICE B}

AUTORISATION DE RECRUTEMENT À LA MAISON DES GREFFÉS DU QUÉBEC 


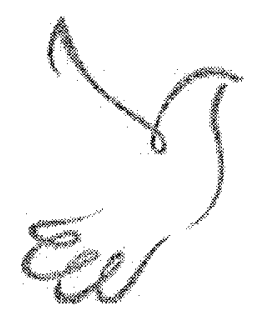

Montréal, le 23 mai 2012

Madame Geneviève Taché Bsc. Inf.

Étudiante à la maîtrise en Sciences infirmières

Université du Québec à Chicoutimi

Objet : Demande d'autorisation de recrutement et d'information sur le déroulement de la collecte de donner auprès des résidents de la Maison des greffés Lina Cyr.

Madame Taché,

Suite à votre demande du 15 mai dernier, je vous confirme que vous pourrez recruter des patients ou patientes de la Maison des greffés Lina Cyr dans le cadre de votre projet de rédaction de votre mémoire sur l'exploration du vécu des patients en attente de transplantation vivant à l'extérieur des grands centres.

Nous collaborerons au recrutement des résidents de la Maison des greffés Lina Cyr pour faire le premier approche auprès d'eux. C'est mon adjointe Renée Brodeau qui collaborera avec vous sur votre dossier, car pour moi j'ai certains dossiers qui me demandent ailleurs. Je tiens à vous remercier d'avoir choisi la Maison des greffés Lina Cyr pour la rédaction de votre mémoire.

Espérant le tout conforme, veuillez agréer, Madame Taché l'expression de mes meilleurs sentiments.

$$
\text { Whelectuie of Qroch }
$$

Micheline Cyr Asselin

Directrice générale

La Maison des greffés Lina Cyr 
APPENDICE C

APPROBATION ÉTHIQUE DE L'UNIVERSITÉ DU QUÉBEC À CHICOUTIMI 


\section{UQAC

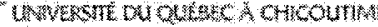

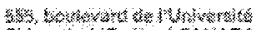 \\ of}

Conite déthique de la recherche

Le 26 jun 2012

Madame Ganeveve Tache

115 ne de Victoria

Chroutin (ouebeo)

GT ANS

NRE 6023520

\section{QBJET: Approbation éthique}

Madame,

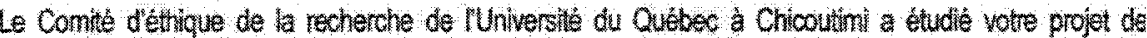

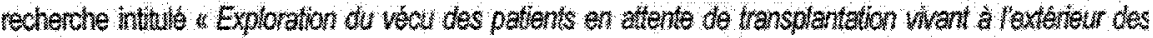

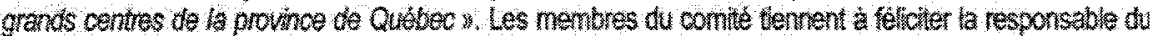
proje ds recherche pour la qualte du dosster presente

Lors de cette wunion, le conte a oxamine les dowments suvants:

$\checkmark$ Le fomulatire Demande daporobation ethique s.

1. Les dedarations dhomeur $(3)$

$\checkmark$ Les oulls de collecte de donnees.

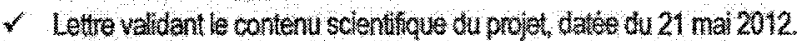

$\checkmark$ Le formulare ditomaton et de consentement.

- Lautorisation de la Mabon des grefes Lina Oy, date du 23 mal 2012

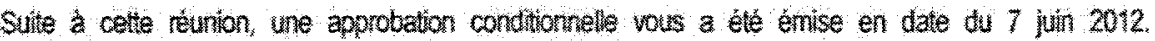
Conomement a nos exigenoes, une version mod do des dowments suvants gete soumise en datu du 21 jun 2012.

$\checkmark$ Le fomulate Denande dapprobalion entique

$\checkmark$ Le fomulaite dinfomaton et de consentemant.

* Le guide dentrovie

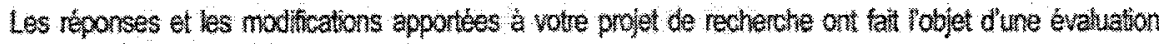
accelére le 26 jum 2012 


\section{Nl est a noter que la preuve dautorisation des organismes nécessaire a la réalisation de votre recherche est toujours manquante et deva être acheminée au CER de VUOAC lorsque cette demière sera émise.}

Nous avons le plaisir de vous infomer que votre projet de recherche a été approuvé par le Comité d'éthique de la recherche. Lapprobation éthique delivé est valide pour la periode du 26 juin 2012 au 30 luin 2013.

Nous vous rappetons quil est de la responsabilté du chercheur de toujours déterit une approbation éthique valide, et ce, tout au long de la recherche. De plus, toute modification au protocole dexperience etiou aux formulaires joints a ce protocole dexpénence doit être approuvee par le Comité d'éthique de la recherche.

Deux mois avant la date d'echéance de votre approbation, vous devrez faire parvenir au comilte une demande de prolongation, en utilisant le document du Comite prévu à cet effet, téléchargeable à partir du sile WEB de linstitution a ladresse sulvante:

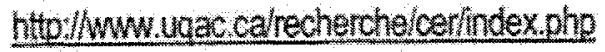

En vous souhaitant le meilleur succès dans la poursute de vos travaux, veullez accepter, Madame, nos salutations distinguees.

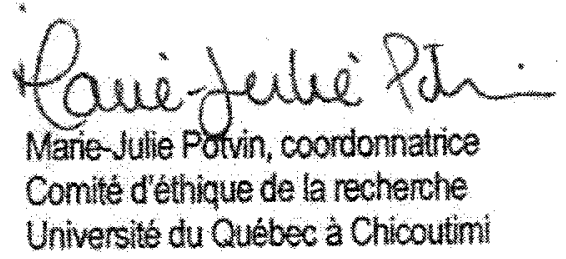

\section{D. i. Aporobation éthique}


APPENDICE D

LETTRE D'INFORMATION SUR LE PROJET DE MÉMOIRE DE MAÎTRISE POUR LES PROFESSIONNELS DU PROGRAMME DE TRANSPLANTATION 
Saguenay, Qué.

Le 15 mai 2012

Objet : Informations sur le projet de mémoire de maitrise pour les professionnelles du programme de transplantation.

Madame, Monsieur,

Je suis étudiante à la maîtrise en sciences infirmières à l'Université du Québec à Chicoutimi et j'en suis à la rédaction de mon mémoire dont le titre est Exploration du vécu des patients en attente de transplantation vivant à l'extérieur des grands centres. Il s'agit d'une étude phénoménologique qui a pour objectif de mieux comprendre la réalité d'être un patient en attente de transplantation. Afin de documenter cette situation, la personne qui la vit est sans contredit la mieux placée pour parler de ce que représente cette pénible expérience.

Quelques études ont démontré que ces patients vivent des moments difficiles, et ce, tant au plan psychologique, que physique et financier. Ces problématiques sont par ailleurs bien souvent majorées par la relocalisation à laquelle ils doivent faire face pour pouvoir recevoir leur organe, puisque le délai alloué pour se rendre au centre de greffe est court. Or, au Québec, les centres transplanteurs sont polarisés dans deux grandes villes, soit Montréal et Québec. Les patients vivant à l'extérieur de ces centres urbains doivent donc trouver un gîte près de leur centre hospitalier transplanteur.

Dans cette situation, la Maison des greffés s'avère l'endroit qui accueille une bonne partie de cette population, du moins à Montréal. C'est pourquoi j'ai sollicité cette maison afin de pouvoir procéder au recrutement des patients faisant partie de mon échantillon, ce qui me faciliterait grandement l'accès à ces personnes qu'il serait fort difficile de joindre autrement.

Le recrutement à la Maison des greffés se fera avec la collaboration de madame Cyr Asselin. Étant donné qu'il est important que les patients se sentent libres de choisir de faire partie de cette étude, une première approche sera faite par cette dernière. Une affiche d'information sur la recherche à venir sera fournie. 
Les participants recherchés pour ce projet sont les patients en attente de transplantation d'organe et ceux ayant reçu une greffe depuis moins de 12 mois. Le sexe, l'âge ainsi que le type d'organe n'ont pas d'importance dans la sélection des participants. Par contre, ils doivent parler et comprendre le français. Une entrevue individuelle semidirigée, d'une durée de plus ou moins 60 minutes, sera réalisée pour faire ressortir leur expérience. Des questions ouvertes leur seront posées afin de leur permettre de s'exprimer sur la réalité qu'ils vivent ou ont vécu et de décrire ce qu'ils pensent. Le guide d'entrevue est joint en annexe.

Une explication plus en profondeur sera fournie au patient avant de débuter l'entrevue et un formulaire de consentement sera signé afin de m'assurer d'une participation éclairée et volontaire. De plus, j'aurai pris contact avec les personnes responsables d'assurer le soutien du patient au programme de greffe de chaque centre transplanteur afin de m'assurer de leur disponibilité pour assurer le suivi psychologique du patient si le besoin s'en fait sentir.

J'espère que cette lettre vous a fourni les renseignements dont vous aurez besoin. Dans le cas contraire, n'hésitez pas à communiquer avec moi et il me fera plaisir de répondre à vos questions.

Je vous remercie à l'avance de votre inestimable soutien à l'avancement de mon projet et vous prie de recevoir l'expression de mes salutations les plus distinguées.

Geneviève Taché Bsc. Inf. Étudiante à la maîtrise en Sciences infïrmières Université du Québec à Chicoutimi

Tel : 418-815-2863

Courriel : genevieve.tache@uqac.ca 
APPENDICE E

AFFICHE DE RECRUTEMENT 


\begin{tabular}{|c|c|}
\hline 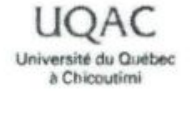 & $\begin{array}{l}\text { Exploration du vécu des patients en attente de } \\
\text { transplantation vivant à l'extérieur des grands centres } \\
\text { ParGenevieve Taché int. } \mathbf{M S c} \text { (c) }\end{array}$ \\
\hline
\end{tabular}

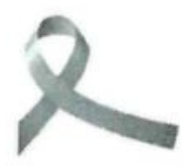

Motif de la recherche

L'artente diune transplantation est une réalité très difficile pour le patient. Celle-ci est méconnue de la population et par plusieurs professionnels de la santé. Cette étude aura donc comme impact de sensibiliser les professionnels et la population à cette réalité. Une meilleure compréhension de cette expérience permettra d'offrir de meilleurs services à ces personnes qui vivent dans I'attente et d'adapter le soutien offert.

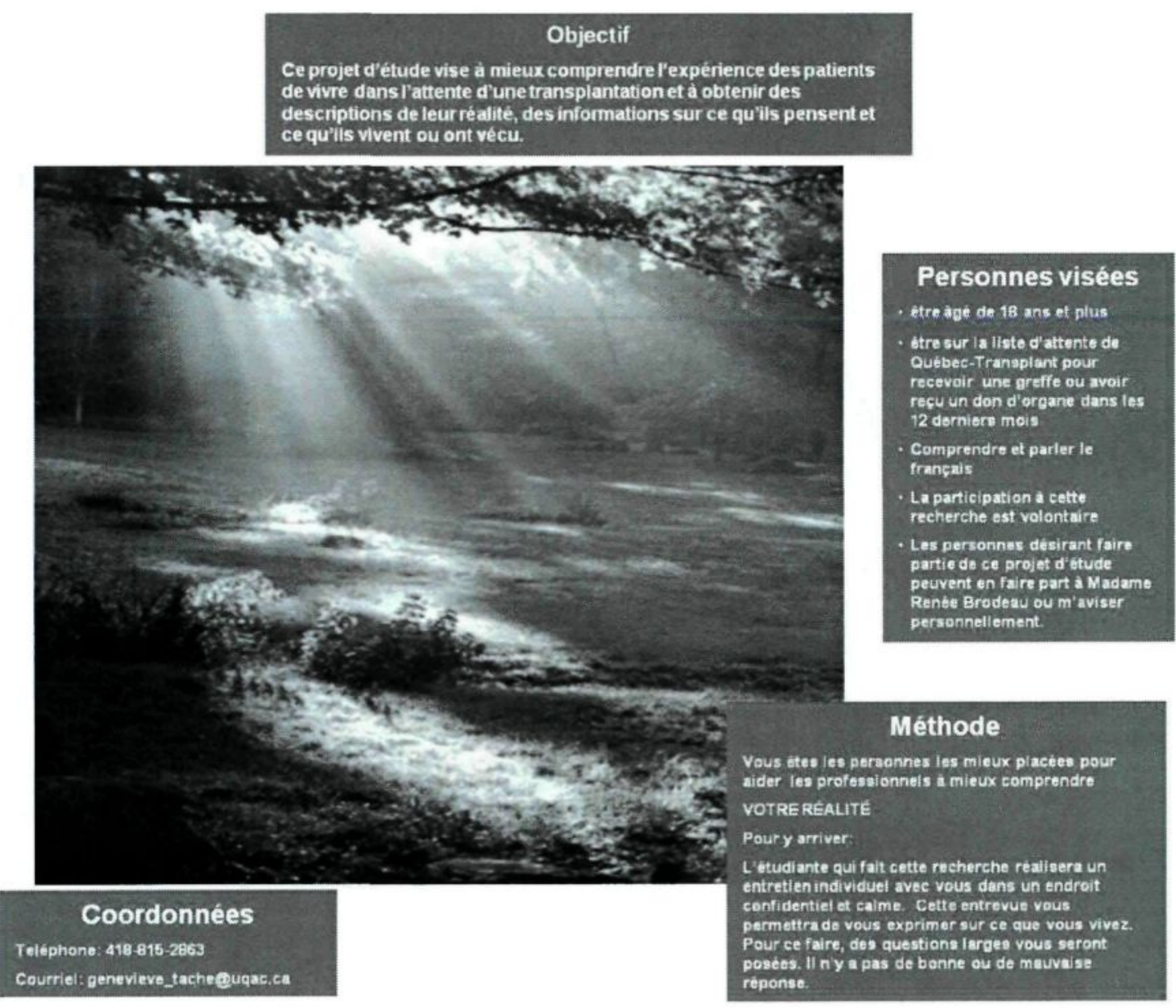

Remerciements

Merci a vous tous a lavance et à madame Asselin davoir accepte que ce projet d'etude soit réalise dans sa maison

Genevieve Tache int:M.Se(c) 
APPENDICE F

LETTRE D'INFORMATION SUR LE PROJET DE MAÎTRISE 
Saguenay, Qué.

\section{UQAC \\ Université du Québec à Chicoutimi}

Le 13 novembre 2012

Objet : Informations sur le projet de maitrise

Madame, Monsieur,

Je suis étudiante à la maîtrise en sciences infirmières à l'Université du Québec à Chicoutimi et j'en suis à la rédaction de mon mémoire dont le titre est Exploration du vécu des patients en attente de transplantation vivant à l'extérieur des grands centres. Il s'agit d'une étude qui a pour objectif de mieux comprendre la réalité d'être un patient en attente de transplantation. Afin de documenter cette situation, la personne qui la vit est sans contredit la mieux placée pour parler de ce que représente cette pénible expérience.

Quelques études ont démontré que ces patients vivent des moments difficiles, et ce, tant au plan psychologique, que physique et financier. Ces problématiques sont par ailleurs bien souvent majorées par la relocalisation à laquelle ils doivent faire face pour pouvoir recevoir leur organe, puisque le délai alloué pour se rendre au centre de greffe est court. Or, au Québec, les centres transplanteurs sont polarisés dans deux grandes villes, soit Montréal et Québec. Les patients vivant à l'extérieur de ces centres urbains doivent

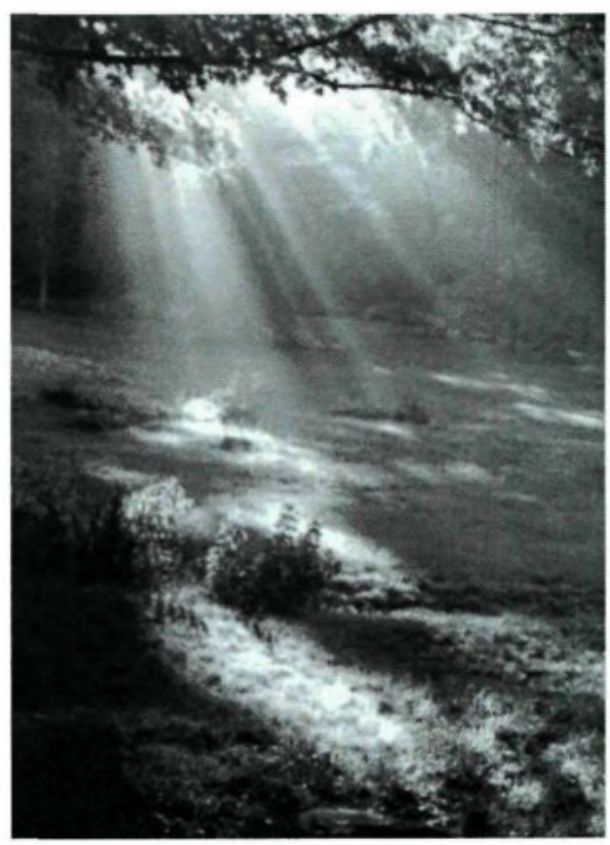
donc trouver un gîte près de leur centre hospitalier transplanteur.

Les participants recherchés pour ce projet sont les patients en attente de transplantation d'organe et ceux ayant reçu une greffe. Le sexe ainsi que le type d'organe n'ont pas d'importance dans la sélection des participants. Par contre, ils doivent parler et 
comprendre le français ainsi qu'être âgés de 18 ans et plus. Une entrevue individuelle semidirigée, d'une durée de plus ou moins 60 minutes, sera réalisée pour faire ressortir leur expérience. Des questions ouvertes seront posées afin de permettre à la personne de s'exprimer sur la réalité qu'elle vit ou a vécue et de décrire ce qu'elle pense. $\mathrm{La}$ participation est volontaire et la personne peut se retirer en tout temps.

J'espère que cette lettre vous a fourni les renseignements dont vous aurez besoin pour prendre une décision éclairée sur votre participation à ce projet de recherche. Dans le cas contraire, ou si vous désirez participer à cette recherche, n'hésitez pas à communiquer avec moi et il me fera plaisir de répondre à vos questions.

Je vous remercie à l'avance de votre inestimable soutien à l'avancement de mon projet et vous prie de recevoir l'expression de mes salutations les plus distinguées.

Geneviève Taché, Bsc. Inf. Étudiante à la maîtrise en Sciences infirmières Université du Québec à Chicoutimi Tel : 418-815-2863 Courriel : genevieve.tache@uqac.ca 
APPENDICE G

QUESTIONNAIRE SOCIODÉMOGRAPHIQUE 


\section{Questionnaire sociodémographique}

1. De quelle ville venez-vous?

2. Depuis combien de temps attendez-vous votre transplantation ou avez-vous attendu?
A. 1-3 mois
B. 4- 6 mois
C. 7-9 mois
D. $10-12$ mois
E. 13 mois et plus

3. Sexe

A. Homme

B. Femme

4. Dans quel groupe d'âge êtes-vous?
A. $18-29$ ans
B. $30-39$ ans
C. $40-49$ ans
D.50 -59 ans
E. 60 ans et plus

5. Quel est votre état matrimonial actuel?
A. Marié(e) ou conjoint(e) de fait
B. Célibataire (jamais marié(e))
C. Divorcé(e) ou séparé(e)
D. Veuf(ve)

\section{Avez-vous des enfants?}

Si oui, combien et quel âge : 
APPENDICE H

GUIDE D'ENTRETIEN 


\section{Guide d'entretien}

Question de recherche : Quelle est la signification de l'expérience d'être un patient en attente de transplantation vivant à l'extérieure des grands centres?

But: explorer le sens de l'expérience d'une personne en attente de transplantation vivant à l'extérieur des grands centres.

Avant de débuter, je tiens à vous remercier d'avoir accepté de participer à mon projet d'étude. Advenant le cas que vous ne voudriez pas répondre à des questions qui seront posées lors de cette entrevue, c'est votre choix et il sera respecté. De plus, il n'y a pas de bonne ou de mauvaise réponse puisque le but de cette étude est de comprendre votre réalité de patient en attente de transplantation. Lors de l'entrevue, si vous êtes fatigué ou si vous ne vous sentez pas bien, nous pouvons reprendre l'entrevue ultérieurement.

- (1) Pour débuter, j'aimerais savoir depuis quand vous avez reçu le diagnostic de votre maladie.

- (2) À partir de quand la greffe est-elle devenue la seule option de traitement?

○ Pouvez-vous m'en dire plus?

- (3) Pouvez-vous me parler du moment où vous avez appris que vous alliez être sur la liste d'attente de Québec-Transplant?

- Qu'avez-vous ressenti?

- Qu'est-ce que vous vous êtes dit dans votre tête?

- (4) Dans le quotidien, pour vous, à quoi ça ressemble d'attendre une transplantation. - Pouvez-vous m'en dire plus? 
- Comment vous sentez-vous face à vos actions?

- (5) Parlez-moi de votre état physique

- Que ressentez-vous face votre état physique?

○ À quel point votre état physique vous affecte?

- (6) Qu'est-ce qui est le plus difficile lorsqu'on attend une transplantation?

- Qu'est-ce qui facilite ou aide votre attente?

- (7) Comment vivez-vous cette attente avec vos proches?

- Quelle influence ont-ils dans l'attente?

- (8) Comment vivez-vous cette attente avec l'équipe de transplantation (Définir équipe)?

- (9) Qu'est-ce que cela veut dire pour vous le mot attente?

- Quel sentiment ressentez-vous face à ce mot?

Je tiens à vous remercier de votre générosité pour avoir participé à cette entrevue et d'avoir répondu aux questions avec le meilleur de vous-même. 
APPENDICE I

FORMULAIRE D'INFORMATION ET DE CONSENTEMENT CONCERNANT LA PARTICIPATION 


\section{FORMULAIRE D'INFORMATION ET DE CONSENTEMENT CONCERNANT LA PARTICIPATION}

\section{TITRE DU PROJET}

Exploration du vécu des patients en attente de transplantation vivant à l'extérieur des grands centres.

\section{RESPONSABLE(S) DU PROJET DE RECHERCHE}

Responsable : Geneviève Taché. Inf. M.sc (candidate)

Directeur de recherche: Suzanne Aucoin, Inf. Ph. D. en sciences infirmières Codirectrice: Édith Ellefsen, inf. Ph. D.

\section{PRÉAMBULE}

Nous sollicitons votre participation à un projet de recherche. Cependant, avant d'accepter de participer à ce projet et de signer ce formulaire d'information et de consentement, veuillez prendre le temps de lire, de comprendre et de considérer attentivement les renseignements qui suivent.

Ce formulaire peut contenir des mots que vous ne comprenez pas. Nous vous invitons à poser toutes les questions que vous jugerez utiles au chercheur responsable du projet ou aux autres membres du personnel affecté au projet de recherche et à leur demander de vous expliquer tout mot ou renseignement qui n'est pas clair. 


\section{NATURE, OBJECTIFS ET DÉROULEMENT DU PROJET DE RECHERCHE}

\subsection{Description du projet de recherche}

Lorsque la décision de procéder à une transplantation d'organe est finalement prise, les personnes auxquelles l'équipe médicale propose cette option vivent depuis de nombreuses années avec une maladie chronique qui est maintenant au stade terminal. La réalité de l'attente d'une transplantation est peu connue des professionnels de la santé et de la population. Pour assurer un suivi et des soins de santé adéquats, les professionnels doivent posséder les connaissances nécessaires. Cependant, pour acquérir ces connaissances, il est important de connaitre le vécu des personnes qui attendent une transplantation. Au Québec, aucune étude n'a encore été faite sur le sujet. Il faut donc aller chercher des informations auprès de ces personnes, afin qu'elles puissent s'exprimer et faire connaître ce qu'elles vivent.

\subsection{Objectif(s) spécifique(s)}

L'objectif de cette étude est d'explorer ce que vit la personne en attente de transplantation, pour augmenter les connaissances et ainsi adapter adéquatement les soins de santé.

\subsection{Déroulement}

Tout d'abord, une entrevue d'une durée approximative d'une heure sera réalisée par l'étudiante, dans un endroit calme et assurant la confidentialité. Avant de débuter l'entrevue, je vais m'assurer que vous avez bien compris le but de cette étude et que vous êtes d'accord pour poursuivre. L'entrevue sera enregistrée sur support audionumérique, afin que je puisse transcrire vos propos plus tard et ne rien oublier. Lors de l'entrevue, je poserai des questions larges sur lesquelles vous pourrez vous exprimer en toute liberté. Il n'y a pas de bonne ou de mauvaise réponse, puisque je veux savoir ce que vous vivez. Advenant que votre condition de santé nécessite un arrêt de 
l'entrevue, elle sera reprise ultérieurement. Par la suite, j'analyserai le contenu des entrevues et je ferai un retour avec vous pour m'assurer que les éléments ressortis représentent bien votre expérience.

\section{AVANTAGES, RISQUES ET/OU INCONVÉNIENTS ASSOCIÉS AU PROJET DE RECHERCHE}

L'avantage de ce projet est que les résultats permettront de sensibiliser les professionnels de la santé et la population à la réalité d'être un patient en attente de transplantation vivant à l'extérieur des grands centres. Une meilleure compréhension de cette expérience permettra d'offrir de meilleurs services et d'adapter le soutien qui leur est offert.

L'inconvénient de ce projet est que, lors de l'entrevue, les questions pourront faire surgir des émotions. Advenant le cas où vous ressentiez le besoin de vous confier à quelqu'un après l'entrevue, la personne-ressource du soutien psychologique de votre équipe de transplantation est avisée de ce projet et est mise à votre disposition. La liste des personnes-ressources de chaque centre transplanteur ainsi que leurs coordonnées est jointe à votre formulaire de consentement.

\section{CONFIDENTIALITÉ DES DONNÉES}

Toutes les données recueillies seront confidentielles et des codes vous seront attribués assurant l'anonymat des informations recueillies et votre possibilité de retrait en tout temps. Les questionnaires et les enregistrements des entrevues seront conservés sous clé dans le bureau de la directrice de maîtrise à l'Université du Québec à Chicoutimi jusqu'en décembre 2012, puisque celle-ci prend sa retraite. Un classeur barré, situé également à l'université, devrait être disponible par la suite, mais cette information est à valider. Autrement, l'étudiante conservera elle-même ses données, dans un classeur 
barré, situé à sa résidence.

Les données informatisées seront conservées sur l'ordinateur portable de l'étudiante et protégées par un code d'accès. Les données seront conservées pour une période de sept ans et détruites par la suite.

\section{PARTICIPATION VOLONTAIRE ET DROIT DE RETRAIT}

Votre participation est entièrement volontaire. Vous pouvez également vous retirer en tout temps de cette étude sans donner de justification. Le refus de participer ou le retrait de l'étude n'entraineront aucune conséquence ou préjudice envers vous.

\section{INDEMNITÉ COMPENSATOIRE}

Vous ne recevrez aucune compensation monétaire pour la participation à cette étude.

\section{PERSONNES-RESSOURCES}

Si vous avez des questions concernant le projet de recherche ou si vous éprouvez un problème que vous croyez relier à votre participation au projet de recherche, vous pouvez communiquer avec le responsable du projet de recherche aux coordonnées suivantes :

Geneviève Taché

Tel. Maison : 418-612-1151

Tel. Cell : 418-815-2863

Courriel : geneviève.tache@uqac.ca

Pour toute question d'ordre éthique concernant votre participation à ce projet de recherche, vous pouvez communiquer avec la coordonnatrice du Comité d'éthique de la recherche aux coordonnées suivantes: 418-545-5011 poste 2493 ou cer@uqac.ca. 


\section{CONSENTEMENT}

\section{Consentement du participant}

J'ai pris connaissance du formulaire d'information et de consentement. Je reconnais qu'on m'a expliqué le projet, qu'on a répondu à mes questions et qu'on m'a laissé le temps voulu pour prendre une décision.

Je consens à participer à ce projet de recherche aux conditions qui y sont énoncées. Une copie signée et datée du présent formulaire d'information et de consentement m'a été remise.

Nom et signature du sujet de recherche

Date

Signature de la personne qui a obtenu le consentement si différent du chercheur responsable du projet de recherche.

J'ai expliqué au sujet de recherche les termes du présent formulaire d'information et de consentement et j'ai répondu aux questions qu'il m'a posées.

Nom et signature de la personne qui obtient le consentement Date

\section{Signature et engagement du chercheur responsable du projet}

Je certifie qu'on a expliqué au sujet de recherche les termes du présent formulaire d'information et de consentement, que l'on a répondu aux questions que le sujet de recherche avait à cet égard et qu'on lui a clairement indiqué qu'il demeure libre de mettre un terme à sa participation, et ce, sans préjudice.

Je m'engage, avec l'équipe de recherche, à respecter ce qui a été convenu au formulaire d'information et de consentement et à en remettre une copie signée au sujet de recherche.

Nom et signature du chercheur responsable du projet de recherche

Date 


\section{APPENDICE J}

ACCEPTATION DU SUIVI PSYCHOLOGIQUE

PAR LES ÉQUIPES DE TRANSPLANTATION 
Le 10 septenthre 2012

\author{
Comite althique \\ Thiversitaire de la recherche \\ Universite du Qublec à Chicoutimi \\ 555 , boulcyard de tothiversite \\ Chicoutimi (Quebe) $\mathrm{G} / \mathrm{H} 2 \mathrm{Bl}$
}

Objet: Projet de recherehe de madume Ganevikwe Tache, Gtudinne a la matrise en sciences intrmières à l Université du Quebec à Chicoutimi

A qui do oroit

Madame Tache a pris contact avec moi dans les derticrs mois ch ce qui a trait a son projet de recherehe potant sur la signification de lexperience d"etre un patienten atente de mansplantaton vixant â l"exterient doc grands cevtres.

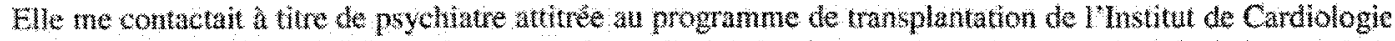

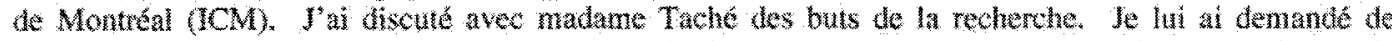

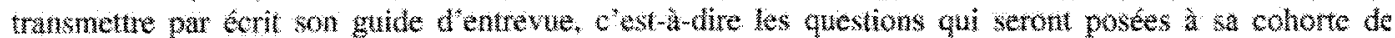
patients en attente d'une grefe cardiaque.

$J^{*}$ at recu par la poste les documents demandes a madame Tache, soit la descripton do son protel di

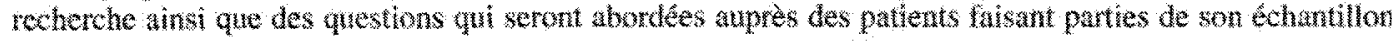
de recherchere.

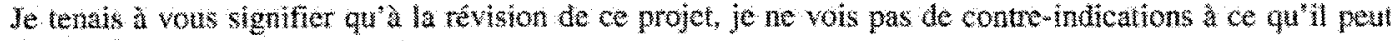
ctra applique en bone ct atue forms.

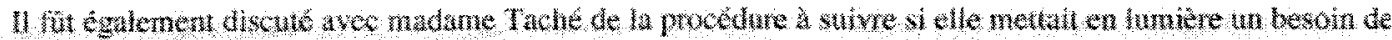
suatien psychologique chez les patients qu' elle rencontretait dans le cadre te sa recherche.

Je lni a bren explque que notre service de médecine psychosomatique situe l' lCM noffre pas de service d'évaluation d"urgence. Elle est au courant tontefois gue notwe service pent ofrir des services et ga"

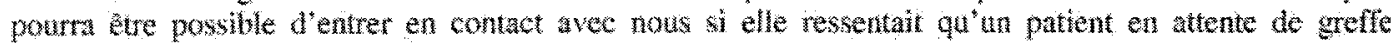

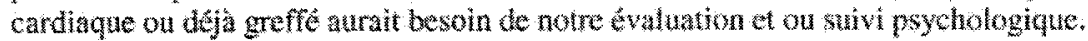

Merci de lattention que vous porterez à cette misstyc.

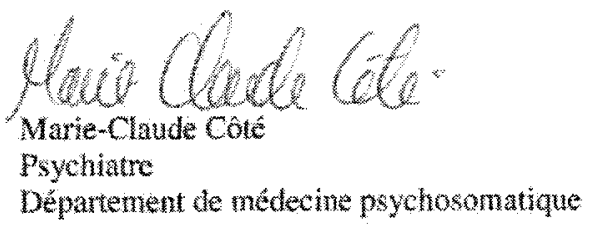


Montréal, le 10 septembre 2012

\section{Bonjour Mme Taché}

Suite à votre demande, je vous confirme que nous offrirons un suivi, au besoin, à nos patients qui participeront à votre projet de mémoire: Exploration du vécu des patients en attente de transplantation vivant à l'extérieur des grands centres.

\section{Cordialement}

\section{Howne Francoeur}

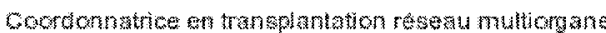

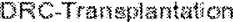

CENTRE WOSPTALER OELUNVERSTE DE WONTREA

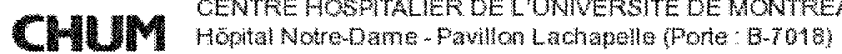

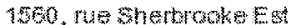

Montreal lourbes H2L 4 WI

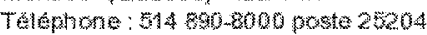

Tolectweur : $514412-7742$

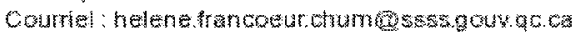

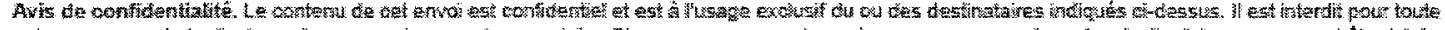

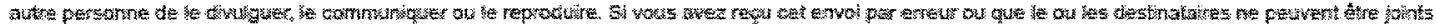

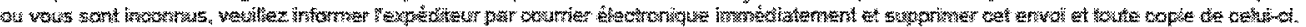

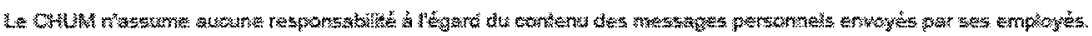


APPENDICE K

RÉSUMÉ DU JOURNAL DE BORD DE L'ÉTUDIANTE CHERCHEURE 


\section{Journal de Bord}

\section{Dévoilement de mon expérience personnelle}

Lors des entretiens, la charge émotive ressentie par les participants a fait ressurgir des souvenirs de l'expérience de transplantation vécue par mon père. Je ressentais le besoin de leur dire : "Je vous comprends ». Cependant, il avait été établi entre ma directrice et moi qu'il était préférable de ne pas révéler que mon père avait été greffé afin de ne pas interférer dans les entretiens. Lors de la dernière rencontre pour les entretiens de validation, certains participants mon demandé pourquoi j'avais choisi ce sujet de recherche. Je leur ai révélé la raison qui avait motivé mon choix seulement à ce moment.

\section{Idée préconçue : difficulté financière}

À prime à bord, je croyais que l'aspect financier serait mis en évidence par les participants. Cependant, à ma grande surprise ils disaient ne pas avoir de problème financier alors j'ai dû mettre cet aspect de côté.

\section{Idée préconçue : attendre à la maison des greffés}

Je croyais au départ que attendre une transplantation était vécu plus difficilement si la personne devait être relocalisée à la maison des greffés. Les entretiens avec les participants qui ont attendu à leur domicile m'ont fait comprendre qu'attendre une transplantation c'est une expérience difficile et similaire, qu'elle soit vécue à sa résidence ou à l'extérieur de chez-soi.

\section{L'omniprésence de la mort}

J'ai été surprise de constater que les participants n'avaient pas peur de la mort, mais bien de quitter ceux qu'ils aiment. Leur famille et amis ont contribué à la prise de décision de tenter la greffe et de poursuivre cette aventure malgré les épreuves rencontrées durant l'attente.

\section{Sentiments vécus lors des entretiens}

Certains entretiens ont été plus difficiles à réaliser que d'autres. Certains patients étaient très émotifs. J'ai trouvé difficile de les voir pleurer et de ne pas pouvoir intervenir.

Lors de l'entrevue de validation avec Hélène, son état de santé était très précaire, ce qui a rendu la validation difficile. Elle avait beaucoup de difficulté à parler et elle devait prendre des calmants pour soulager ses douleurs. Une fois l'entretien terminé, je n'ai pu m'empêcher de pleurer dans ma voiture, car sa situation m'a énormément touchée.

\section{Expérience personnelle}

Il est certain que j'ai fait abstraction de mon vécu personnel lors des entretiens. Cependant, lors de l'analyse, certains verbatim m'ont beaucoup touchée et ont soulevé des sentiments qui étaient enfouis depuis près de 8 ans. 


\section{APPENDICE L}

ACCEPTATION DE MODIFICATION PAR LE COMITÉ D'ÉTHIQUE DE LA RECHERCHE DE L'UNIVERSITÉ DU QUÉBEC À CHICOUTIMI 


\section{UQAC

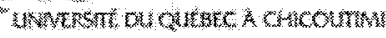

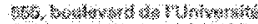

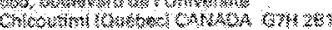

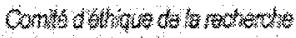

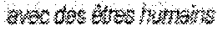 \\ Le 15 nowenbre 2012 \\ Madane Gendenèe Taché \\ 15 ne de Vorotia \\ Chiouth (Quebec) \\ G764N8}

NRË 602.35201

\section{OBJET . Modification d"un projet de recherche}

Madrma

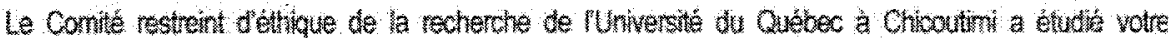

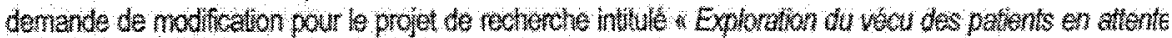

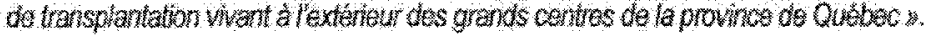

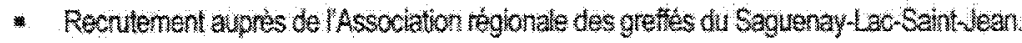

Le fou ayan cte luge satistasant nous awoxs le plasir de wous inomer que la modficalion oropose pou

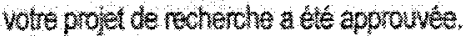

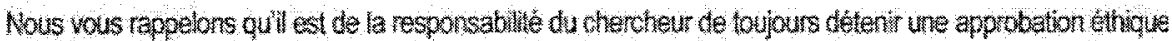

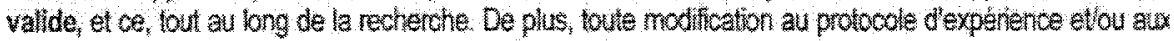

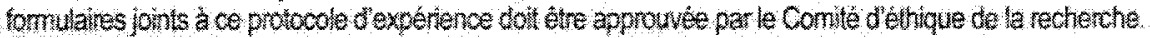

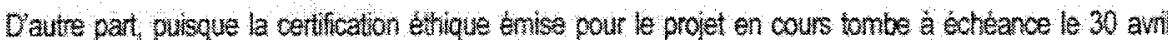

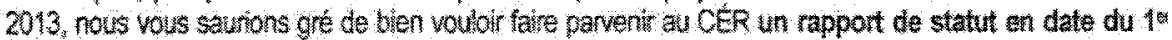

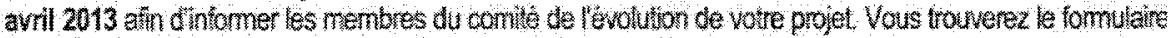

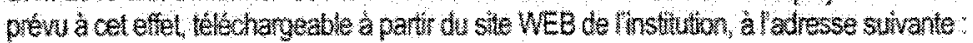

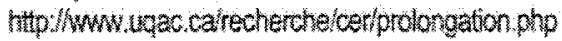

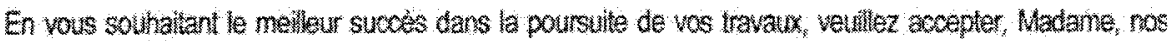
salutations distingueses.

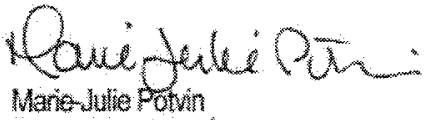

Coordonatios CER 